\title{
Arylation and Alkenylation of Activated Alkyl Halides using Sulfonamides
}

DOI:

10.1039/DOCCOO22OH

Document Version

Accepted author manuscript

Link to publication record in Manchester Research Explorer

\section{Citation for published version (APA):}

Greaney, M., Johnson, S., \& Kovács, E. (2020). Arylation and Alkenylation of Activated Alkyl Halides using Sulfonamides. Chemical Communications, 56(21), 3222-3224. https://doi.org/10.1039/D0CC00220H

\section{Published in:}

Chemical Communications

\section{Citing this paper}

Please note that where the full-text provided on Manchester Research Explorer is the Author Accepted Manuscript or Proof version this may differ from the final Published version. If citing, it is advised that you check and use the publisher's definitive version.

\section{General rights}

Copyright and moral rights for the publications made accessible in the Research Explorer are retained by the authors and/or other copyright owners and it is a condition of accessing publications that users recognise and abide by the legal requirements associated with these rights.

\section{Takedown policy}

If you believe that this document breaches copyright please refer to the University of Manchester's Takedown Procedures [http://man.ac.uk/04Y6Bo] or contact uml.scholarlycommunications@manchester.ac.uk providing relevant details, so we can investigate your claim.

\section{OPEN ACCESS}




\section{Arylation and Alkenylation of Activated Alkyl Halides using Sulfonamides}

Stuart Johnson, Ervin Kovács and Michael F. Greaney*

School of Chemistry, University of Manchester, Oxford Road, Manchester, UK, M13 9PL, UK

\section{Supporting Information}

$\begin{array}{lr}\text { 1. General remarks } & 2\end{array}$

2. Synthesis of Arylsulfonamides 3

2.1 N-Alkyl Sulfonamide Syntheses 4

2.2 N-Aryl Sulfonamide Syntheses $\quad 6$

$\begin{array}{lr}\text { 2.3 Phenylethenesulfonamide Syntheses } & 10\end{array}$

3. Sulfonamide Coupling Reactions with Smiles Rearrangement 12

$\begin{array}{ll}\text { 3.1 Compound Data for Smiles products } & 14\end{array}$

4. Reduction of Imine to Arylglycine Derivative 29

$\begin{array}{lr}\text { 5. NMR Spectra } & 31\end{array}$

$\begin{array}{ll}\text { 6. References } & 65\end{array}$ 


\section{General remarks}

${ }^{1} \mathrm{H}$ NMR, ${ }^{13} \mathrm{C}\left\{{ }^{1} \mathrm{H}\right\}$ NMR and ${ }^{19} \mathrm{~F}$ NMR were recorded at 500/400 MHz, 126/101 MHz, $470 / 376 \mathrm{MHz}$ on Bruker Avance 500 or 400 spectrometers. All ${ }^{1} \mathrm{H}$ NMR and ${ }^{13} \mathrm{C}$ chemical shifts were referenced to the residual solvent peak of $\mathrm{CDCl}_{3}\left({ }^{1} \mathrm{H}\right.$ referenced to $7.26 \mathrm{ppm}$ and ${ }^{13} \mathrm{C}$ referenced to $\left.77.16 \mathrm{ppm}\right),\left(\mathrm{CD}_{3}\right)_{2} \mathrm{SO}\left({ }^{1} \mathrm{H}\right.$ referenced to $2.50 \mathrm{ppm}$ and ${ }^{13} \mathrm{C}$ referenced to $39.52 \mathrm{ppm}$ ), methanol ( ${ }^{1} \mathrm{H}$ referenced to $3.31 \mathrm{ppm}$ and ${ }^{13} \mathrm{C}$ referenced to $49.00 \mathrm{ppm}$ ), $\mathrm{CD}_{3} \mathrm{CN}$ ( ${ }^{1} \mathrm{H}$ referenced to $1.94 \mathrm{ppm}$ and ${ }^{13} \mathrm{C}$ referenced to $1.32 \mathrm{ppm}$ ), and acetone ( ${ }^{1} \mathrm{H}$ referenced to $2.05 \mathrm{ppm}$ and ${ }^{13} \mathrm{C}$ referenced to $29.84 \mathrm{ppm}$ ). All ${ }^{19} \mathrm{~F}$ chemical shifts were unadjusted from raw data. All chemical shifts are quoted in parts per million (ppm), measured from the centre of the signal except in the case of multiplets, which are quoted as a range. Coupling constants are quoted to the nearest $0.1 \mathrm{~Hz}$. Splitting patterns are abbreviated as follows: singlet (s), doublet $(\mathrm{d})$, triplet $(\mathrm{t})$, quartet (q), quintet (quin), sextet (sxt), multiplet (m), broad singlet (br. s) and combinations thereof. Assignment of spectra was aided by DEPT 135 and 2D NMR spectroscopy (COSY, HSQC and HMBC). Assignments are provided in the following format: chemical shift (multiplicity, coupling constant, integration, description of functional group, letter referenced to molecule drawn above).

Low resolution mass spectrometry was performed on an Agilent 6100 mass spectrometer (ESI ionisation) and Hewlett Packard 5971 MSD (GC/MS with EI). High resolution mass spectrometry was performed on a Waters QTOF with ESI/APCI ionisation and a Thermo Finnigan MAT95XP (EI).

Melting points were determined using a Kofler hot-stage apparatus and are uncorrected.

Thin layer chromatography (TLC) was performed on commercially available precoated TLC plates (Merck Silica gel 6o F254 aluminium sheets). Visualisation was either achieved under UV light at $254 \mathrm{~nm}$ or with a $\mathrm{KMnO}_{4}$ stain.

Column chromatography was conducted on silica gel (Sigma Aldrich, 40-63 $\mu \mathrm{m}$, 6o Å) or Biotage KP-Sil or Snap Ultra cartridges on a Biotage Isolera automated columning machine. 


\section{Synthesis of Arylsulfonamides}

General Procedure A for the synthesis of $N$-methyl arylsulfonamides

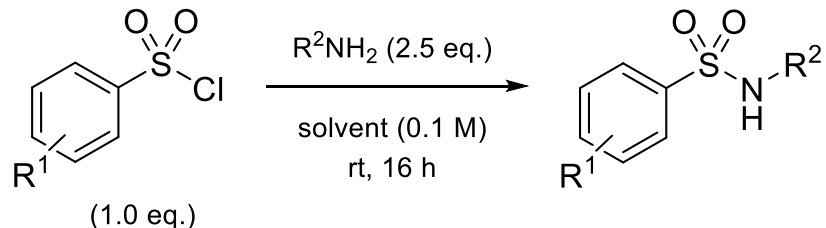

To a solution of sulfonyl chloride (1.0 eq.) in diethyl ether or ethyl acetate (o.10 $\mathrm{m})$, alkylamine (2.5 eq.) was added dropwise. The reaction mixture was stirred for 16 hours at room temperature, then it was concentrated under reduced pressure. $\mathrm{HCl}$ (1.0 $\mathrm{M}$ ) was added, the organic compounds were extracted with DCM. The combined organic layers were washed with $\mathrm{H}_{2} \mathrm{O}$ and brine, dried over $\mathrm{MgSO}_{4}$, filtered and concentrated under reduced pressure to give the crude product, which was purified by column chromatography on silica gel.

General Procedure B for the synthesis of $N$-phenyl arylsulfonamides<smiles>[R][R]1ccc(NS(=O)(=O)c2ccc([N+](=O)[O-])cc2)cc1</smiles>

To a solution of substituted aniline (1.0 eq.) in freshly distilled THF (o.10 M), pyridine (1.2 eq.) and 4-nitrobenzenesulfonyl chloride (1.2 eq.) were added at $\mathrm{o}{ }^{\circ} \mathrm{C}$. The reaction mixture was allowed to warm to room temperature and stirred for 16 hours. The reaction was quenched with aqueous saturated $\mathrm{NH}_{4} \mathrm{Cl}$. The organic layers were extracted with EtOAc, washed with $\mathrm{H}_{2} \mathrm{O}$ and brine, dried over $\mathrm{MgSO}_{4}$, filtered and concentrated under reduced pressure to give the crude product, which was purified by column chromatography on silica gel. 


\subsection{N-Alkyl Sulfonamide Syntheses}

\section{4-nitro- $N$-methylbenzenesulfonamide $6 a$}

Prepared according to General Procedure A from $4^{-}$
(30 wt $\%$ in $\mathrm{EtOH}, 11 \mathrm{mmol}, 1.17 \mathrm{~mL}), \mathrm{Et}_{2} \mathrm{O}(45.0 \mathrm{~mL})$. Purification by column chromatography on silica gel (o-40\% EtOAc in hexanes) afforded 6a as a pale yellow solid (888 mg, $4.1 \mathrm{mmol}$, 91\%), m.p. $93-95{ }^{\circ} \mathrm{C}$.

${ }^{1} \mathrm{H}$ NMR (400 MHz, $\mathrm{CDCl}_{3}$ ): $\delta 8.41$ (app. d, $J=9.0 \mathrm{~Hz}, 2 \mathrm{H}, \mathrm{CH}_{\mathrm{ar}}-2$ ), 8.09 (app. d, $J=9.0$ $\left.\mathrm{Hz}, 2 \mathrm{H}, \mathrm{CH}_{\mathrm{ar}}-3\right), 4.67(\mathrm{q}, J=5.2 \mathrm{~Hz}, 1 \mathrm{H}, \mathrm{NH}), 2.76\left(\mathrm{~d}, J=5.2 \mathrm{~Hz}, 3 \mathrm{H}, \mathrm{CH}_{3}-5\right) ;{ }^{13} \mathrm{C} \mathbf{N M R}$ $\left(101 \mathrm{MHz}, \mathrm{CDCl}_{3}\right): \delta 150.3(\mathrm{C}-1), 145.0(\mathrm{C}-4), 128.6(\mathrm{C}-3), 124.6(\mathrm{C}-2), 29.5(\mathrm{C}-5)$.

IR (neat film, $\mathrm{cm}^{-1}$ ): 3302, 3107, 2985, 1733, 1536, 1383, 1354, 1174, 854, 739.

MS (ES ${ }^{-}$) found $m / z 215[\mathrm{M}-\mathrm{H}]^{-}$; HRMS (ES ${ }^{-}$) found 215.0110, $\mathrm{C}_{7} \mathrm{H}_{7} \mathrm{~N}_{2} \mathrm{O}_{4} \mathrm{~S}[\mathrm{M}-\mathrm{H}]^{-}$ requires 215.0121 .

Data is in accordance with literature reports. ${ }^{[1]}$

2-nitrobenzene- $N$-methyl-sulfonamide $6 b$

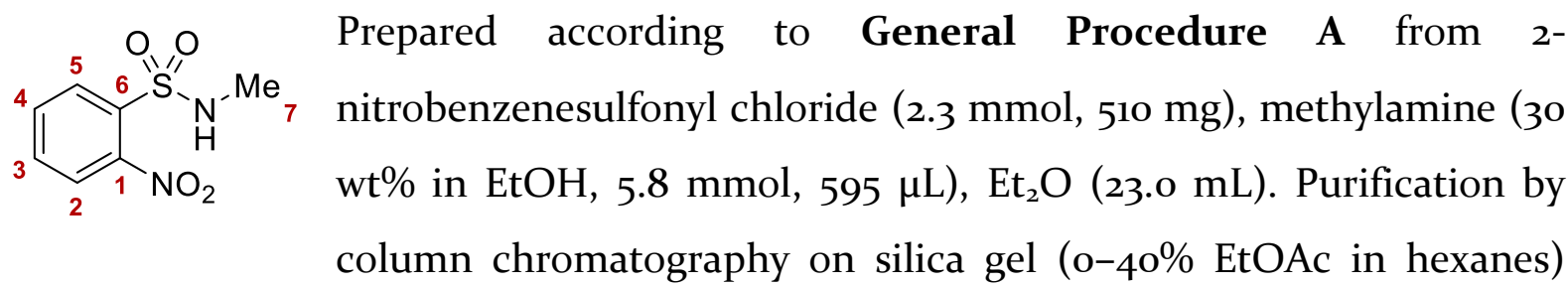

afforded $\mathbf{6 b}$ as a white solid (218 $\mathrm{mg}$, $1.0 \mathrm{mmol}, 44 \%$ ).

${ }^{1} \mathrm{H}$ NMR (400 MHz, CD $\left.\mathrm{CN}\right): \delta$ 8.16-8.11 (m, $\left.1 \mathrm{H}, \mathrm{CH}_{\mathrm{ar}}-5\right), 7.90-7.85\left(\mathrm{~m},{ }_{1} \mathrm{H}, \mathrm{CH}_{\mathrm{ar}}-2\right)$, 7.78-7.73 (m, 2H, $\left.\mathrm{CH}_{\mathrm{ar}-3}, 4\right), 5.23$ (br. s, $\left.1 \mathrm{H}, \mathrm{NH}\right), 2.79\left(\mathrm{~d}, \mathrm{~J}=5.3 \mathrm{~Hz}\right.$ ); ${ }^{13} \mathrm{C}$ NMR (101 $\left.\mathrm{MHz}, \mathrm{CDCl}_{3}\right): \delta 148.4(\mathrm{C}-1), 133.8(\mathrm{C}-4), 132.8$ (C-3), 132.6 (C-6), 131.7 (C-5), 125.6 (C-2), $29.9(\mathrm{C}-7)$.

Data is in accordance with literature reports. ${ }^{[2]}$ 
4-nitro-3-(trifluoromethyl)- $N$-methylbenzenesulfonamide $6 \mathrm{c}$<smiles>CNS(=O)(=O)c1ccc([N+](=O)[O-])c(C(F)(F)F)c1</smiles>

Prepared according to General Procedure A from 4-nitro-3(trifluoromethyl)benzenesulfonyl chloride (0.92 mmol, $263 \mathrm{mg}$ ), methylamine (30 wt\% in EtOH, $2.3 \mathrm{mmol}, 239 \mu \mathrm{L}), \mathrm{Et}_{2} \mathrm{O}$ (10.0 $\mathrm{mL}$ ). Purification by column chromatography on silica gel (o40\% EtOAc in hexanes) afforded 6c as a dark brown solid (225 mg, o.79 mmol, 86\%), m.p. $81-84^{\circ} \mathrm{C}$.

${ }^{1} \mathrm{H}$ NMR (400 MHz, $\left.\mathrm{CDCl}_{3}\right): \delta 8.33\left(\mathrm{~d}, J=1.9 \mathrm{~Hz},{ }_{1} \mathrm{H}, \mathrm{CH}_{\mathrm{ar}^{-}}{ }^{-5}\right), 8.25(\mathrm{dd}, J=8.4,1.9 \mathrm{~Hz}$, $\left.{ }_{1} \mathrm{H}, \mathrm{CH}_{\mathrm{ar}}-3\right), 8.04\left(\mathrm{~d}, J=8.4 \mathrm{~Hz}, 1 \mathrm{H}, \mathrm{CH}_{\mathrm{ar}}-2\right), 4.65(\mathrm{q}, J=5.2 \mathrm{~Hz}, 1 \mathrm{H}, \mathrm{NH}), 2.8 \mathrm{o}(\mathrm{d}, J=5.2$ $\left.\mathrm{Hz}, 3 \mathrm{H}, \mathrm{CH}_{3}-8\right)$; ${ }^{13} \mathrm{C}$ NMR (101 MHz, $\mathrm{CDCl}_{3}$ ): $\delta 150.2(\mathrm{C}-1), 143.9(\mathrm{C}-4), 132.2(\mathrm{C}-3), 127.2-$ 127.1 (m, C-5), 126.3 (q, J=22.6 Hz, C-6), 126.1 (C-2), 123.8 (q, J=225.5 Hz, C-7), 29.5 (C8); ${ }^{19}$ F NMR (376 MHz, $\left.\mathrm{CDCl}_{3}\right): \delta-60.16$.

IR (neat film, $\mathrm{cm}^{-1}$ ): 3307, 3114, 3089, 3043, 2770, 1614, 1543, 1307, 1291, 836, 639.

MS (ES ${ }^{-}$) found $m / z 283[\mathrm{M}-\mathrm{H}]^{-}$; HRMS $\left(\mathrm{ES}^{-}\right)$found 283.0005, $\mathrm{C}_{8} \mathrm{H}_{6} \mathrm{~F}_{3} \mathrm{~N}_{2} \mathrm{O}_{4} \mathrm{~S}[\mathrm{M}-\mathrm{H}]^{-}$ requires 282.9995 .

\section{4-nitro-2-methoxy- $N$-methylbenzenesulfonamide $6 \mathrm{~d}$}

Prepared according to General Procedure A from 4-nitro-2-<smiles>CNS(=O)(=O)c1ccc([N+](=O)[O-])cc1OC</smilesmethoxybenzenesulfonyl chloride (1.0 mmol, $256 \mathrm{mg})$, methylamine (30 wt\% in $\mathrm{EtOH}, 2.6 \mathrm{mmol}, 269 \mu \mathrm{L}), \mathrm{Et}_{2} \mathrm{O}$ (10.0 $\mathrm{mL}$ ). Purification by column chromatography on silica gel (o40\% EtOAc in hexanes) afforded 6d as a clear yellow solid (237 mg, $0.96 \mathrm{mmol}, 94 \%$ ), m.p. $169-172{ }^{\circ} \mathrm{C}$.

${ }^{1}$ H NMR (40o MHz, $\left.\mathrm{CDCl}_{3}\right): \delta 8.13\left(\mathrm{~d}, J=8.5 \mathrm{~Hz}, 1 \mathrm{H}, \mathrm{CH}_{\mathrm{ar}}{ }^{-3}\right), 7.97(\mathrm{dd}, J=8.5$, 2.o Hz, $\left.{ }_{1} \mathrm{H}, \mathrm{CH}_{\mathrm{ar}}-2\right), 7.91\left(\mathrm{~d}, J=2.0 \mathrm{~Hz}, 1 \mathrm{H}, \mathrm{CH}_{\mathrm{ar}}-6\right), 4.92(\mathrm{q}, J=5.5 \mathrm{~Hz}, 1 \mathrm{H}, \mathrm{NH}), 4.13(\mathrm{~s}, 3 \mathrm{H}$, $\left.\mathrm{CH}_{3}-7\right), 2.67\left(\mathrm{~d}, J=5.5,3 \mathrm{H}, \mathrm{CH}_{3}-8\right) ;{ }^{13} \mathrm{C} \mathrm{NMR}\left(101 \mathrm{MHz}, \mathrm{CDCl}_{3}\right): \delta 156.9(\mathrm{C}-1), 151.7(\mathrm{C}-4)$, 132.4 (C-3), 132.0 (C-2), 115.8 (C-6), 107.5 (C-5), 57.4 (C-7), 29.6 (C-8).

IR (neat film, $\mathrm{cm}^{-1}$ ): 3310, 3108, 2925, 2853, 1717, 1406, 1313, 1265, 1164, 1025, 802, 611. 
MS (ES ${ }^{-}$) found $m / z$ 245 $[\mathrm{M}-\mathrm{H}]^{-}, 247[\mathrm{M}+\mathrm{H}]^{+}$; HRMS (ES ${ }^{-}$) found 245.0239, $\mathrm{C}_{8} \mathrm{H}_{9} \mathrm{~N}_{2} \mathrm{O}_{5} \mathrm{~S}[\mathrm{M}-\mathrm{H}]^{-}$requires 245.0227.

\subsection{N-Aryl Sulfonamide Syntheses}

\section{4-nitro- $N$-phenylbenzenesulfonamide $6 \mathrm{e}$}

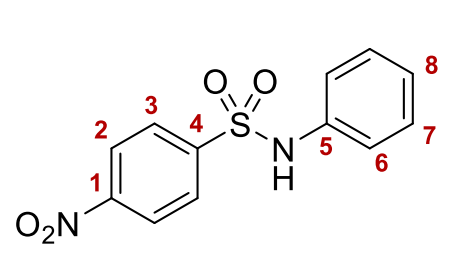

Prepared according to General Procedure A from 4nitrobenzenesulfonyl chloride $147 \mathrm{a}(4.5 \mathrm{mmol}$, $1.00 \mathrm{mg})$, aniline (11 mmol, $1.03 \mathrm{~mL})$, EtOAc (45.0 mL). Purification by column chromatography on silica gel (o-40\% EtOAc in hexanes) afforded $6 \mathbf{e}$ as a light pink solid (834 mg, $3.0 \mathrm{mmol}, 67 \%)$, m.p. $157-159{ }^{\circ} \mathrm{C}$.

${ }^{1} \mathrm{H}$ NMR (50o MHz, $\mathrm{CDCl}_{3}$ ): $\delta 8.28$ (app. d, $J=8.5 \mathrm{~Hz}, 2 \mathrm{H}, \mathrm{CH}_{\mathrm{ar}}-2$ ), 7.92 (app. d, $J=8.5$ $\left.\mathrm{Hz}, 2 \mathrm{H}, \mathrm{CH}_{\mathrm{ar}}-3\right), 7.31-7.25\left(\mathrm{~m}, 2 \mathrm{H}, \mathrm{CH}_{\mathrm{ar}}-7\right), 7.21-7.17\left(\mathrm{~m},{ }_{1} \mathrm{H}, \mathrm{CH}_{\mathrm{ar}}-8\right)$, 7.09-7.06 (m, $2 \mathrm{H}$, $\left.\mathrm{CH}_{\mathrm{ar}}-6\right), 6.68$ (br. s, $1 \mathrm{H}, \mathrm{NH}$ ); ${ }^{13} \mathrm{C}$ NMR (126 MHz, $\left.\mathrm{CDCl}_{3}\right): \delta 150.4(\mathrm{C}-1), 144.8(\mathrm{C}-4)$, 135.4 (C-5), 129.8 (C-6), 128.7 (C-3), 126.7 (C-8), 124.4 (C-2), 122.6 (C-7).

IR (neat film, $\mathrm{cm}^{-1}$ ): 3275, 3133, 2922, 2857, 1918, 1608, 1520, 1400, 1332, 1163, 854, 739, $669,616$.

MS $\left(\mathrm{ES}^{-}\right.$) found $m / z 277[\mathrm{M}-\mathrm{H}]^{-}$; HRMS $\left(\mathrm{ES}^{-}\right)$found 277.0281, $\mathrm{C}_{12} \mathrm{H}_{9} \mathrm{~N}_{2} \mathrm{O}_{4} \mathrm{~S}[\mathrm{M}-\mathrm{H}]^{-}$ requires 277.0289 .

Data is in accordance with literature reports. ${ }^{[3]}$

4-nitro- $N$-(p-tolyl)benzenesulfonamide $6 f$

4-nitrobenzenesulfonyl chloride (1.2 mmol, $265 \mathrm{mg}), \mathrm{THF}$
toluidine (1.0 mmol, $107 \mathrm{mg})$, pyridine $(1.2 \mathrm{mmol}, 35 \mathrm{~L})$, (10.0 mL). Purification by column chromatography on silica gel (o-40\% EtOAc in hexanes) afforded 6 f as a yellow solid (214 mg, $0.73 \mathrm{mmol}, 73 \%)$, m.p. $176-178^{\circ} \mathrm{C}$.

${ }^{1} \mathrm{H}$ NMR (40o MHz, CD 3 CN): $\delta 8.26$ (app. d, $J=9.0 \mathrm{~Hz}, 2 \mathrm{H}, \mathrm{CH}_{\mathrm{ar}}-2$ ), 8.01 (br. s, $1 \mathrm{H}$, $\mathrm{NH}$ ), 7.91 (app. d, J=9.0 Hz, 2H, $\mathrm{CH}_{\mathrm{ar}}-3$ ), 7.08 (app. d, $J=8.4 \mathrm{~Hz}, 2 \mathrm{H}, \mathrm{CH}_{\mathrm{ar}}-6$ ), 6.97 (d, $\left.J=8.4 \mathrm{~Hz}, 2 \mathrm{H}, \mathrm{CH}_{\mathrm{ar}^{-}} 7\right), 2.24\left(\mathrm{~s}, 3 \mathrm{H}, \mathrm{CH}_{3}-9\right) ;{ }^{13} \mathrm{C}$ NMR (101 MHz, $\left.\mathrm{CD}_{3} \mathrm{CN}\right): \delta 151.1(\mathrm{C}-4)$, 
145.8 (C-1), 136.8 (C-8), 134.7 (C-5), 130.9 (C-6), 129.5 (C-3), 125.3 (C-2), 123.3 (C-7), 20.2 (C-9).

IR (neat film, $\mathrm{cm}^{-1}$ ): 3269, 3106, 3039, 2920, 2866, 1607, 1528, 1509, 1348, 1313, 1302, 1162, 1090, 855, 745, 736, 683, 643, 607 .

MS $\left(\mathrm{ES}^{+}\right)$found $m / z 337[\mathrm{M}+2 \mathrm{Na}-\mathrm{H}]^{+}$; HRMS $\left(\mathrm{ES}^{+}\right)$found 337.0225, $\mathrm{C}_{13} \mathrm{H}_{11} \mathrm{~N}_{2} \mathrm{O}_{4} \mathrm{SNa}_{2}$ $[\mathrm{M}+2 \mathrm{Na}-\mathrm{H}]^{+}$requires 337.0229 .

Data is in accordance with literature reports. ${ }^{[4]}$

4-nitro- $N$-mesitylbenzenesulfonamide $6 \mathrm{~g}$<smiles>Cc1cc(C)c(NS(=O)(=O)c2ccc([N+](=O)[O-])cc2)c(C)c1</smiles>

Prepared according to General Procedure B from 2,4,6trimethylaniline (1.0 mmol, $141 \mu \mathrm{L})$, pyridine $(1.2 \mathrm{mmol}$, $35 \mu \mathrm{L}$ ), 4-nitrobenzenesulfonyl chloride (1.2 mmol, 265 $\mathrm{mg})$, THF (10.0 mL). Purification by column chromatography on silica gel (o-40\% EtOAc in hexanes) afforded $\mathbf{6 g}$ as a light brown solid (220 mg, o.69 mmol, 69\%), m.p. $144-146^{\circ} \mathrm{C}$.

${ }^{1} \mathrm{H}$ NMR (40o MHz, CD $\mathrm{CN}$ ): $\delta 8.34$ (app. d, $J=8.9 \mathrm{~Hz}, 2 \mathrm{H}, \mathrm{CH}_{\mathrm{ar}}-2$ ), 7.94 (app. d, $J=$ $8.9 \mathrm{~Hz}, 2 \mathrm{H}, \mathrm{CH}_{\mathrm{ar}}-3$ ), 7.43 (br. s, $1 \mathrm{H}, \mathrm{NH}$ ), 6.90 (s, $\left.2 \mathrm{H}, \mathrm{CH}_{\mathrm{ar}}-7\right), 2.26$ (s, $\left.{ }_{3} \mathrm{H}, \mathrm{CH}_{3}-10\right), 1.97$ (s, 6H, $\left.\mathrm{CH}_{3}-9\right)$ ) ${ }^{13} \mathrm{C}$ NMR (101 MHz, $\left.\mathrm{CD}_{3} \mathrm{CN}\right): \delta 151.2(\mathrm{C}-4), 148.2(\mathrm{C}-1)$, $139.0(\mathrm{C}-8), 138.8$ (C-6), 130.7 (C-5), 130.3 (C-7), 129.2 (C-3), 125.5 (C-2), 20.9 (C-10), 18.9 (C-9).

IR (neat film, $\mathrm{cm}^{-1}$ ): 3281, 3105, 2924, 2862, 1524, 1349, 1311, 1163, 1091, 854, 738, 685, 622.

MS $\left(\mathrm{ES}^{-}\right.$) found $\mathrm{m} / \mathrm{z} 319$ [M-H] $^{-}$; HRMS (ES ${ }^{-}$) found 319.0754, $\mathrm{C}_{15} \mathrm{H}_{15} \mathrm{~N}_{2} \mathrm{O}_{4} \mathrm{~S}[\mathrm{M}-\mathrm{H}]^{-}$ requires 319.0758 .

Data is in accordance with literature reports. ${ }^{[5]}$ 


\section{4-nitro- $N$-(4-methoxyphenyl)benzenesulfonamide $6 \mathrm{~h}$}

Prepared according to General Procedure B from $p$-anisidine (1.0 mmol, $123 \mathrm{mg}$ ),<smiles>COc1ccc(NS(=O)(=O)c2ccc([N+](=O)[O-])cc2)cc1</smiles>
pyridine (1.2 mmol, $35 \mu \mathrm{L})$, 4-nitrobenzenesulfonyl chloride (1.2 mmol, $265 \mathrm{mg})$, THF (10.0 mL). Purification by column chromatography on silica gel (o-40\% EtOAc in hexanes) afforded $\mathbf{6 h}$ as a light yellow solid (18o mg, $0.59 \mathrm{mmol}, 58 \%)$, m.p. $174-176^{\circ} \mathrm{C}$.

${ }^{1} \mathrm{H}$ NMR (400 MHz, CD $\mathrm{CN}$ ): $\delta 8.26$ (app. d, $J=9.0 \mathrm{~Hz}, 2 \mathrm{H}, \mathrm{CH}_{\mathrm{ar}}-2$ ), 7.90 (br. s, $1 \mathrm{H}$, $\mathrm{NH}$ ), 7.85 (app. d, $J=9.0 \mathrm{~Hz}, 2 \mathrm{H}, \mathrm{CH}_{\mathrm{ar}}-3$ ), 6.98 (app. d, $J=9.1 \mathrm{~Hz}, 2 \mathrm{H}, \mathrm{CH}_{\mathrm{ar}}-7$ ), $6.8 \mathrm{o}$ (app. d, J = 9.1 Hz, 2H, CHar-6), 3.72 (s, $\left.3 \mathrm{H}, \mathrm{CH}_{3}-9\right) ;{ }^{13} \mathrm{C}$ NMR (101 MHz, CD $\mathrm{CN}$ ): $\delta 159.2$ (C-8), 152.3 (C-4), 145.7 (C-1), 129.7 (C-5), 129.5 (C-3), 126.3 (C-7), 125.3 (C-2), 115.4 (C-6), $56.1(\mathrm{C}-9)$.

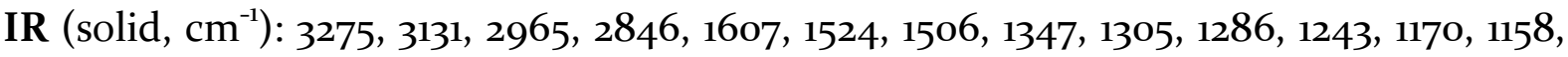
1087, 1030, 852, 750, 739, 68o, 66o.

MS (ES ${ }^{-}$) found $m / z 307[\mathrm{M}-\mathrm{H}]^{-}$; HRMS (ES ${ }^{-}$) found 307.0389, $\mathrm{C}_{13} \mathrm{H}_{11} \mathrm{~N}_{2} \mathrm{O}_{5} \mathrm{~S}[\mathrm{M}-\mathrm{H}]^{-}$ requires 307.0394.

Data is in accordance with literature reports. ${ }^{[6]}$

\section{Ethyl 4-((4-nitrophenyl)sulfonamido)benzoate 6i}

Prepared according to General Procedure B from<smiles>CCOC(=O)c1ccc(NS(=O)(=O)c2ccc([N+](=O)[O-])cc2)cc1</smiles>
p-aminoethylbenzoate (1.o mmol, $165 \mathrm{mg})$, pyridine (1.2 mmol, $35 \mu \mathrm{L}$ ), 4-nitrobenzenesulfonyl chloride (1.2 mmol, $265 \mathrm{mg}$ ), THF (10.0 mL). Purification by column chromatography on silica gel $(0-40 \%$

EtOAc in hexanes) afforded $\mathbf{6 i}$ as a light salmon pink solid (112 mg, $0.32 \mathrm{mmol}, 32 \%$ ), m.p. $180-184^{\circ} \mathrm{C}$.

${ }^{1} \mathrm{H}$ NMR (400 MHz, $\left(\mathrm{CD}_{3}\right)_{2} \mathrm{CO}$ ): $\delta 8.40$ (app. d, $J=8.9 \mathrm{~Hz}, 2 \mathrm{H}, \mathrm{CH}_{\mathrm{ar}}-2$ ), 8.14 (app. d, $J=$ $8.9 \mathrm{~Hz}, 2 \mathrm{H}, \mathrm{CH}_{\mathrm{ar}}-3$ ), 7.92 (app. d, $J=8.8 \mathrm{~Hz}, 2 \mathrm{H}, \mathrm{CH}_{\mathrm{ar}}-7$ ), 7.36 (app. d, $J=8.8 \mathrm{~Hz}, 2 \mathrm{H}$, $\left.\mathrm{CH}_{\mathrm{ar}}-6\right), 4.29$ (q, J = 7.2 Hz, $\left.2 \mathrm{H}, \mathrm{CH}_{2}-10\right), 2.83$ (br. s, $\left.1 \mathrm{H}, \mathrm{NH}\right), 1.32\left(\mathrm{t}, J=7.2 \mathrm{~Hz},{ }_{3} \mathrm{H}, \mathrm{CH}_{3}-\right.$ 
11) ; ${ }^{13} \mathrm{C}$ NMR (101 MHz, $\left.\left(\mathrm{CD}_{3}\right)_{2} \mathrm{CO}\right): \delta$ 166.o (C-9), 145.9 (C-1), $142.3(\mathrm{C}-4), 132.0(\mathrm{C}-5)$, 131.7 (C-7), 129.5 (C-3), 127.5 (C-8), 125.4 (C-2), 120.3 (C-6), 61.4 (C-10), 14.5 (C-11).

IR (neat film, cm ${ }^{-1}$ ): 3228, 3107, 2983, 1712, 1691, 1607, 1531, 1368, 1349, 1132, 1280, 1165, 1109, 1090, 855, 736, 609, 605.

MS (ES ${ }^{-}$) found $m / z 349$ [M-H $^{-}$; HRMS (ES ${ }^{-}$) found 349.0495, $\mathrm{C}_{15} \mathrm{H}_{13} \mathrm{~N}_{2} \mathrm{O}_{6} \mathrm{~S}[\mathrm{M}-\mathrm{H}]^{-}$ requires 349.0500 .

\section{4-nitro- $N$-benzylbenzenesulfonamide $6 \mathbf{j}$}

Prepared according to General Procedure A from 4-<smiles>O=[N+]([O-])c1ccc(S(=O)(=O)NCc2ccccc2)cc1</smilesnitrobenzenesulfonyl chloride $(4.5 \mathrm{mmol}$, $1.00 \mathrm{~g})$, benzylamine (11 mmol, $1.23 \mathrm{~mL})$, EtOAc (45.0 $\mathrm{mL})$.

Purification by column chromatography on silica gel (o$40 \%$ EtOAc in hexanes) afforded $\mathbf{6 j}$ as a yellow solid (1.07 g, $3.7 \mathrm{mmol}, 81 \%)$, m.p. 111$113{ }^{\circ} \mathrm{C}$.

${ }^{1} \mathrm{H}$ NMR (50o $\mathrm{MHz}, \mathrm{CDCl}_{3}$ ): $\delta 8.30$ (app. d, $J=8.4 \mathrm{~Hz}, 2 \mathrm{H}, \mathrm{CH}_{\mathrm{ar}}-2$ ), 7.98 (app. d, $J=8.4$ $\left.\mathrm{Hz}, 2 \mathrm{H}, \mathrm{CH}_{\mathrm{ar}}-3\right)$, 7.28-7.24 (m, $\left.3 \mathrm{H}, \mathrm{CH}_{\mathrm{ar}}-7,9\right), 7.18-7.15\left(\mathrm{~m}, 2 \mathrm{H}, \mathrm{CH}_{\mathrm{ar}}-8\right)$, 5.06 (t, $J=6.1$ $\mathrm{Hz}, 1 \mathrm{H}, \mathrm{NH}), 4.22\left(\mathrm{~d}, J=6.1 \mathrm{~Hz}, 2 \mathrm{H}, \mathrm{CH}_{2}-5\right) ;{ }^{13} \mathrm{C} \mathrm{NMR}\left(126 \mathrm{MHz}, \mathrm{CDCl}_{3}\right): \delta 150.1(\mathrm{C}-1)$, 146.2 (C-4), 135.6 (C-6), 129.0 (C-8), 128.44 (C-3), 128.41 (C-9), 128.0 (C-7), 124.4 (C-2), $47 \cdot 5(\mathrm{C}-5)$.

IR (neat film, $\mathrm{cm}^{-1}$ ): 3289, 3099, 1520, 1421, 1347, 1330, 1312, 1153, 1051, 806, 754, 747, 736, 621.

MS $\left(\mathrm{ES}^{-}\right)$found $m / z 291[\mathrm{M}-\mathrm{H}]^{-}$; HRMS $\left(\mathrm{ES}^{-}\right.$) found 291.0441, $\mathrm{C}_{13} \mathrm{H}_{11} \mathrm{~N}_{2} \mathrm{O}_{4} \mathrm{~S}[\mathrm{M}-\mathrm{H}]^{-}$ requires 291.0445 .

Data is in accordance with literature reports. ${ }^{[7]}$ 


\subsection{Phenylethenesulfonamide Syntheses}

(E)-4-Nitrophenylethenesulfonamide $13 \mathrm{a}^{[8]}$<smiles>CO[N+](=O)c1ccc(/C=C/S(N)(=O)=O)cc1</smiles>

In a $100 \mathrm{~mL}$ Schenk flask under nitrogen atmosphere, $\mathbf{S}$ tert-butyl (((diphenylphosphoryl)methyl)sulfonyl)carbamate (1.0 eq., $1.0 \mathrm{mmol}, 395 \mathrm{mg}$ ) was dissolved in dry DMF (25.0 mL) and cooled to $0^{\circ} \mathrm{C}$. To a well-stirred solution of the reagent $\mathrm{NaH}$ (55\% in mineral oil, 2.6 eq., $2.6 \mathrm{mmol}$, $111 \mathrm{mg}$ ) was added. The reaction was allowed to warm to r.t. and stirred for 30 minutes. Cooled down to o ${ }^{\circ} \mathrm{C}, 4^{-}$ nitrobenzaldehyde (1.2 eq., $1.2 \mathrm{mmol}, 185 \mathrm{mg}$ ) was then added in ten portions and the reaction mixture (dark purple) was stirred vigoursly overnight at room temperature. Distilled water (50.0 mL), EtOAc (40.0 mL) and $2 \%$ aq. $\mathrm{HCl}(10.0 \mathrm{~mL})$ were added to the mixture and the layers were separated. The aqueous layer was extracted with EtOAc $(4 \times 20 \mathrm{~mL})$. The organic solution was washed with distilled water $(2 \times 30 \mathrm{~mL})$, brine $(2 \times 30 \mathrm{~mL})$, and dried over sodium sulfate, filtered and concentrated under reduced pressure to give the crude product (590 mg dark brown oil). The crude mixture was purified by column chromatography (10-60\% EtOAc in hexanes). The Boc protected $\mathbf{S 2}$ compound was obtained as a yellow solid (220 $\mathrm{mg}$, $0.69 \mathrm{mmol}$, 69\% yield.

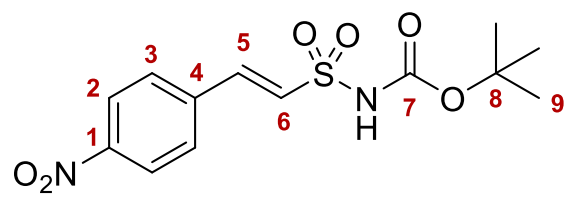

${ }^{1} \mathrm{H}$ NMR (400 MHz, $\left.\left(\mathrm{CD}_{3}\right)_{2} \mathrm{CO}\right): \delta 9.99$ (br. s, $1 \mathrm{H}$, $\mathrm{NH}), 8.33\left(\mathrm{~d}, J=8.9 \mathrm{~Hz}, 2 \mathrm{H}, \mathrm{CH}_{\mathrm{ar}}-2\right), 8.07(\mathrm{~d}, J=8.9$ $\left.\mathrm{Hz}, 2 \mathrm{H}, \mathrm{CH}_{\mathrm{ar}-3}\right), 7.73(\mathrm{~d}, J=15.6 \mathrm{~Hz}, 1 \mathrm{H}, \mathrm{CH}-5), 7.53$ $(\mathrm{d}, J=15.6 \mathrm{~Hz}, 1 \mathrm{H}, \mathrm{CH}-6), 1.44\left(\mathrm{~s}, 9 \mathrm{H}, \mathrm{CH}_{3}-9\right) ;{ }^{13} \mathrm{C} \mathrm{NMR}\left(101 \mathrm{MHz},\left(\mathrm{CD}_{3}\right)_{2} \mathrm{CO}\right): \delta 149.9$ (C-1), 149.1 (C-4), 140.3 (C-5), 138.9 (C-6), 129.7 (C-2), 124.1 (C-3), 82.5 (C-8), 27.2 (C-9). HRMS $\left(\mathrm{APCI}^{-}\right) \mathrm{C}_{13} \mathrm{H}_{15} \mathrm{~N}_{2} \mathrm{O}_{6} \mathrm{~S}^{-}\left([\mathrm{M}-\mathrm{H}]^{-}\right)$requires 327.0656; found 327.0657 .

Boc-protected sulfonamide (S2, $2.1 \mathrm{mmol}, 689 \mathrm{mg})$ was dissolved in DMSO (6.0 $\mathrm{mL})$ and stirred for 8 hours at $80^{\circ} \mathrm{C}$. The reaction mixture was cooled to room temperature, 
poured into distilled water $(40.0 \mathrm{~mL})$ and extracted with EtOAc $(4 \times 20 \mathrm{~mL})$. The organic solution was concentrated under reduced pressure to give the crude product (650 mg). The crude mixture was purified by column chromatography (20-80\% EtOAc in hexanes) to afford 13a as a yellow solid (345 $\mathrm{mg}, 1.5 \mathrm{mmol}, 72 \%$ yield).<smiles>NS(=O)(=O)/C=C/c1ccc([N+](=O)[O-])cc1</smiles>

${ }^{1} \mathbf{H}$ NMR (400 MHz, $\left.\left(\mathrm{CD}_{3}\right)_{2} \mathrm{CO}\right): \delta 8.30(\mathrm{~d}, J=8.8 \mathrm{~Hz}, 2 \mathrm{H}$, $\mathrm{CH}_{\mathrm{ar}-2}$ ), $7.97\left(\mathrm{~d}, J=8.8 \mathrm{~Hz}, 2 \mathrm{H}, \mathrm{CH}_{\mathrm{ar}}-3\right), 7.52(\mathrm{~d}, J=15.6 \mathrm{~Hz}$, $\left.{ }_{1} \mathrm{H}, \mathrm{CH}-5\right), 7.43\left(\mathrm{~d}, J=15.6 \mathrm{~Hz},{ }_{1} \mathrm{H}, \mathrm{CH}-6\right), 6.51$ (br. s, $2 \mathrm{H}, \mathrm{NH}_{2}$ ); ${ }^{13} \mathrm{C}$ NMR (101 MHz, $\left.\left(\mathrm{CD}_{3}\right)_{2} \mathrm{CO}\right): \delta 140.9(\mathrm{C}-1), 135.8$ (C-5), 134.8 (C-4 and C-6), $130.1(\mathrm{C}-$ 3), $124.9(\mathrm{C}-2)$. HRMS $\left(\mathrm{APCI}^{+}\right)$found 229.0274, $\mathrm{C}_{8} \mathrm{H}_{9} \mathrm{~N}_{2} \mathrm{O}_{4} \mathrm{~S}[\mathrm{M}+\mathrm{H}]^{+}$requires 229.0278 .

\section{(E)-N-Methyl-(4-nitrophenyl)ethenesulfonamide $13 \mathbf{b}^{[9]}$}

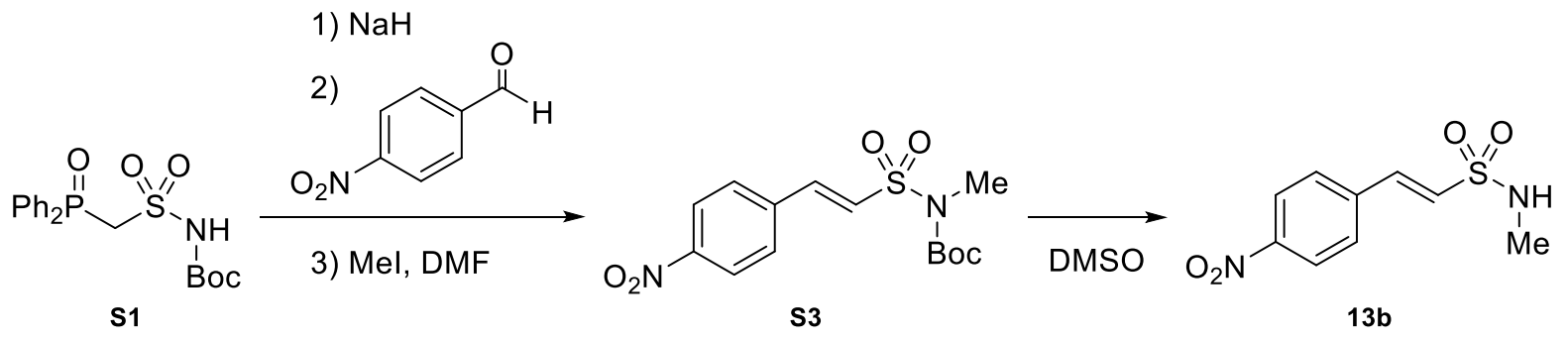

In a $100 \mathrm{~mL}$ Schenk flask under nitrogen atmosphere, S1 tert-butyl (((diphenylphosphoryl)methyl)sulfonyl)carbamate (1.o eq., $1.3 \mathrm{mmol}, 494 \mathrm{mg}$ ) was dissolved in dry DMF (35.0 mL) and cooled to o ${ }^{\circ} \mathrm{C}$. To a well-stirred solution of the reagent $\mathrm{NaH}$ (55\% in mineral oil, 2.6 eq., $3.2 \mathrm{mmol}, 139 \mathrm{mg}$ ) was added in five portions. The reaction was allowed to warm to r.t. and stirred for 30 minutes. Cooled down to $\mathrm{o}{ }^{\circ} \mathrm{C}$, 4-nitrobenzaldehyde (1.2 eq., $1.5 \mathrm{mmol}, 231 \mathrm{mg}$ ) was then added in three portions and the reaction mixture (dark purple) was stirred vigoursly overnight (16 hours) at r.t. Cooled to o ${ }^{\circ} \mathrm{C}$, MeI (5.o eq., $6.3 \mathrm{mmol}$, $0.390 \mathrm{~mL}$ ) was added, stirred at $40{ }^{\circ} \mathrm{C}$ for 4 hours. Ice and EtOAc (50.0 mL) were added to the reaction and the layers were separated. The aqueous layer was extracted with EtOAc $(4 \times 50 \mathrm{~mL})$. The organic solution was washed with $2 \%$ aq. $\mathrm{HCl}(50.0 \mathrm{~mL})$, distilled water $(2 \times 100 \mathrm{~mL})$, brine $(2 \times$ $100 \mathrm{~mL}$ ), and dried over sodium sulfate, filtered and concentrated under reduced pressure to give the crude product (610 mg dark brown oil). The crude mixture was purified by column chromatography (10-35\% EtOAc in hexanes). The compound $\mathbf{S}_{3}$ was obtained as an orange solid (259 $\mathrm{mg}, 0.757 \mathrm{mmol}, 61 \%$ yield). 
<smiles>CN(C(=O)OC(C)(C)C)S(=O)(=O)C=Cc1ccc([N+](=O)[O-])cc1</smiles>

10); ${ }^{13} \mathrm{C}$ NMR (101 MHz, $\left.\left(\mathrm{CD}_{3}\right)_{2} \mathrm{CO}, \mathrm{ppm}\right): \delta 152.1$ (C-8), 149.9 (C-1), $141.0(\mathrm{C}-4), 139.7(\mathrm{C}-$ 5), 130.6 (C-2), 130.2 (C-6), 125.0 (C-3), 84.6 (C-9), 33.1 (C-7), 28.1 (C-10). HRMS (APCI $\left.{ }^{+}\right)$ found 343.0952, $\mathrm{C}_{14} \mathrm{H}_{19} \mathrm{~N}_{2} \mathrm{O}_{6} \mathrm{~S}[\mathrm{M}+\mathrm{H}]^{+}$requires 343.0958 .

The Boc-sulfonamide (S3, 0.75 mmol, $256 \mathrm{mg}$ ) was dissolved in DMSO (3.75 $\mathrm{mL}$ ) and heated to $120{ }^{\circ} \mathrm{C}$ for 8 hours. The reaction mixture was cooled to r.t., poured into distilled water $(35.0 \mathrm{~mL})$ and extracted with EtOAc $(3 \times 30 \mathrm{~mL})$. The organic solution was concentrated under reduced pressure to give the crude product ( $240 \mathrm{mg}$ ). The crude mixture was purified by column chromatography (10-80\% EtOAc in hexanes). The title compound $\mathbf{1 3 b}$ was obtained as an orange solid (153 mg, $0.632 \mathrm{mmol}, 84 \%$ yield).

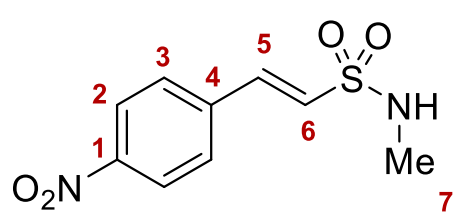

${ }^{1} \mathrm{H}$ NMR (400 MHz, $\left.\left(\mathrm{CD}_{3}\right)_{2} \mathrm{CO}\right): \delta 8.30(\mathrm{~d}, J=8.8 \mathrm{~Hz}, 2 \mathrm{H}$, $\left.\mathrm{CH}_{\mathrm{ar}}-2\right)$, $8.00\left(\mathrm{~d}, J=8.8 \mathrm{~Hz}, 2 \mathrm{H}, \mathrm{CH}_{\mathrm{ar}}-3\right), 7.52(\mathrm{~d}, J=15.6 \mathrm{~Hz}$, $1 \mathrm{H}, \mathrm{CH}-5), 7.29(\mathrm{~d}, J=15.6 \mathrm{~Hz}, 1 \mathrm{H}, \mathrm{CH}-6), 6.24$ (br. s, $1 \mathrm{H}$, $\mathrm{NH}), 2.72\left(\mathrm{~s}, 3 \mathrm{H}, \mathrm{CH}_{3}-7\right) ;{ }^{13} \mathrm{C}$ NMR (101 MHz, $\left.\left(\mathrm{CD}_{3}\right)_{2} \mathrm{CO}\right): \delta$ 149.6 (C-1), 140.6 (C-4), 138.5 (C-5), 130.8 (C-6), 130.2 (C-2), 124.9 (C-3), 28.8 (C-7). HRMS $\left(\mathrm{APCI}^{+}\right)$found $242.0367, \mathrm{C}_{9} \mathrm{H}_{10} \mathrm{~N}_{2} \mathrm{O}_{4} \mathrm{~S}[\mathrm{M}]^{+}$requires 242.0361.

\section{Sulfonamide Coupling Reactions with Smiles Rearrangement}

\section{General Procedure E}

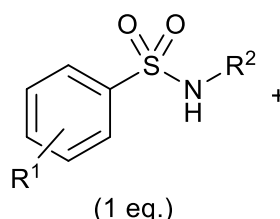

(1 eq.)<smiles>[R]C(Cl)C(=O)OCC</smiles>

(2 eq.)

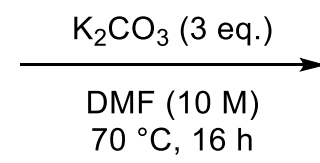

$70{ }^{\circ} \mathrm{C}, 16 \mathrm{~h}$

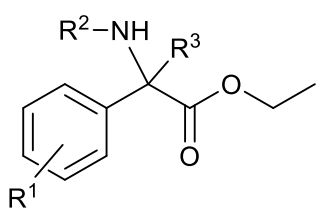

In a microwave vial dried in the oven, sulfonamide (1.0 eq.), potassium carbonate (3.o eq.) were weighted. A stirrer bar was added, the vial was sealed and the air was evacuated then the vial was filled with nitrogen. Dry DMF (о.10 м for sulfonamide), 
substituted ethyl 2-chloroacetate (2.0 eq.) was added to a stirred mixture and the vial was put in a pre-warmed $70{ }^{\circ} \mathrm{C}$ oil bath to stir for 16 hours. The reaction was cooled, the vial was opened, ethyl acetate and water were added and the layers were separated. The aqueous layer was extracted with ethyl acetate. The combined organic layers were then washed three times with a $10 \%$ aqueous $\mathrm{LiCl}$ solution, brine, and dried over magnesium sulfate, filtered and concentrated under reduced pressure to give the crude product. The crude mixture was purified by column chromatography on silica gel.

\section{General Procedure F}

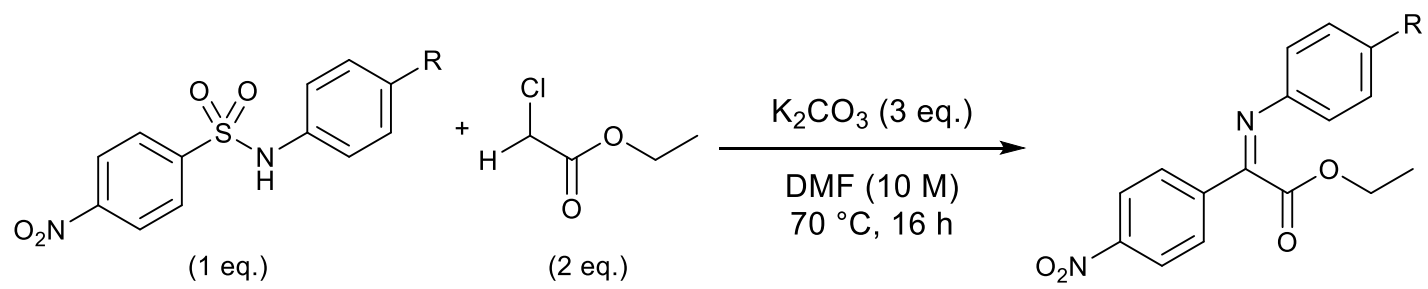

To a solution of an $\mathrm{N}$-aryl sulfonamide (1.o eq.), $\mathrm{K}_{2} \mathrm{CO}_{3}$ (3.0 eq.), DMF (о.10 м for sulfonamide) within a metal-capped, oven-dried microwave vial, ethyl 2-chloroacetate (2.o eq.) was added. The vial was put in a pre-warmed $70{ }^{\circ} \mathrm{C}$ oil bath to stir for 16 hours. The reaction was cooled, the vial was opened, ethyl acetate and water were added and the layers were separated. The aqueous layer was extracted with ethyl acetate. The combined organic layers were then washed three times with a $10 \%$ aqueous $\mathrm{LiCl}$ solution, brine, and dried over magnesium sulfate, filtered and concentrated under reduced pressure to give the crude product. The crude mixture was purified by column chromatography on silica gel. 


\subsection{Compound Data for Smiles products}

Ethyl 2-(methylamino)-2-(4-nitrophenyl)-2-phenylacetate 8a

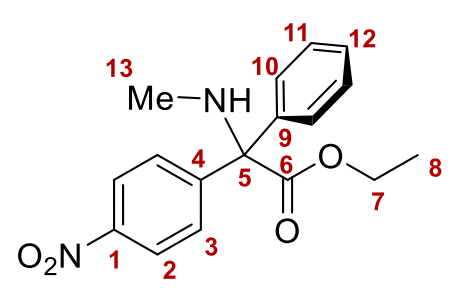

Prepared according to General Procedure E from 4-nitro$N$-methylbenzenesulfonamide 6a (0.10 mmol, $21.6 \mathrm{mg}$ ), $\mathrm{K}_{2} \mathrm{CO}_{3}$ (0.30 mmol, $41.5 \mathrm{mg}$ ), ethyl $\alpha$-chlorophenylacetate 7a (o.20 mmol, $52 \mu \mathrm{L}$ ), DMF (1.00 mL). Purification by column chromatography on silica gel (o-40\% EtOAc in hexanes) afforded 8a as a light yellow solid (87.o mg, $0.28 \mathrm{mmol}$, 92\%); m.p. 53-55 ${ }^{\circ} \mathrm{C}$.

${ }^{1} \mathrm{H}$ NMR (400 MHz, $\mathrm{CDCl}_{3}$ ): $\delta 8.16$ (app. d, $J=9.0 \mathrm{~Hz}, 2 \mathrm{H}, \mathrm{CH}_{\mathrm{ar}}-2$ ), 7.73 (app. d, $J=9.0$ $\left.\mathrm{Hz}, 2 \mathrm{H}, \mathrm{CH}_{\mathrm{ar}}-3\right)$, 7.39-7.27 (m, $\left.5 \mathrm{H}, \mathrm{CH}_{\mathrm{ar}}-10,11,12\right), 4.32-4.21\left(\mathrm{~m}, 2 \mathrm{H}, \mathrm{CH}_{2}-7\right), 2.34$ (br. s, $1 \mathrm{H}, \mathrm{NH}), 2.10\left(\mathrm{~s}, 3 \mathrm{H}, \mathrm{CH}_{3}-13\right), 1.15\left(\mathrm{t}, J=7.2 \mathrm{~Hz}, 3 \mathrm{H}, \mathrm{CH}_{3}-8\right) ;{ }^{13} \mathrm{C}$ NMR (101 $\left.\mathrm{MHz}, \mathrm{CDCl}_{3}\right)$ : $\delta 172.6$ (C-6), 148.6 (C-4), 147.1 (C-1), 140.7 (C-9), 129.7 (C-3), 128.5 (C-10), 128.0 (C-12), 127.9 (C-11), 123.1 (C-2), 73.2 (C-5), 62.1 (C-7), 30.9 (C-13), 14.1 (C-8).

IR (neat film, $\mathrm{cm}^{-1}$ ): 3349, 3063, 2981, 2804, 1821, 1728, 1510, 1320, 1223, 1211, 1158, 1026, $853,734,701,633$.

MS $\left(\mathrm{ES}^{+}\right)$found $m / z 315[\mathrm{M}+\mathrm{H}]^{+}$; HRMS $\left(\mathrm{ES}^{+}\right.$) found 315.1336, $\mathrm{C}_{17} \mathrm{H}_{19} \mathrm{~N}_{2} \mathrm{O}_{4}[\mathrm{M}+\mathrm{H}]^{+}$ requires 315.1326 .

Ethyl 2-amino-2-(4-nitrophenyl)-2-phenylacetate 8b

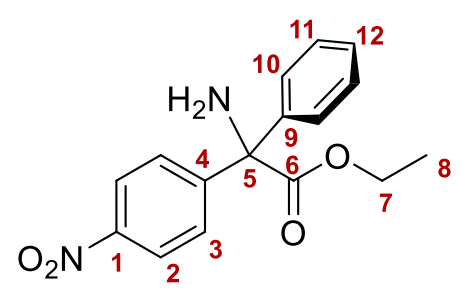

Prepared according to General Procedure E from 4-nitrobenzenesulfonamide ( $0.30 \mathrm{mmol}, 66.5 \mathrm{mg}), \mathrm{K}_{2} \mathrm{CO}_{3}(0.90$ mmol, $124 \mathrm{mg}$ ), ethyl $\alpha$-chlorophenylacetate $7 \mathrm{a}$ (o.6o mmol, $103 \mu \mathrm{L})$, DMF (3.00 $\mathrm{mL})$. Purification by column chromatography on silica gel (o-40\% EtOAc in hexanes) afforded $\mathbf{8 b}$ as a clear colourless oil (13.5 mg, 0.045 mmol, 15\%).

${ }^{1} \mathrm{H}$ NMR (400 MHz, $\mathrm{CDCl}_{3}$ ): $\delta 8.09$ (app. d, $J=9.0 \mathrm{~Hz}, 2 \mathrm{H}, \mathrm{CH}_{\mathrm{ar}}-2$ ), 7.74 (app. d, $J=9.0$ $\mathrm{Hz}, 2 \mathrm{H}, \mathrm{CH}_{\mathrm{ar}}-3$ ), 7.29-7.24 (m, $\left.3 \mathrm{H}, \mathrm{CH}_{\mathrm{ar}}-10,12\right)$, 7.23-7.19 (m, $\left.2 \mathrm{H}, \mathrm{CH}_{\mathrm{ar}}-11\right)$, 4.25-4.18 (m, $\left.2 \mathrm{H}, \mathrm{CH}_{2}-7\right), 2.30$ (br. s, $\left.2 \mathrm{H}, \mathrm{NH}_{2}\right), 1.17\left(\mathrm{t}, J=7.2 \mathrm{~Hz}, 3 \mathrm{H}, \mathrm{CH}_{3}-8\right) ;{ }^{13} \mathrm{C}$ NMR $(101 \mathrm{MHz}$, 
$\left.\mathrm{CDCl}_{3}\right): \delta 173.8$ (C-6), 150.8 (C-1), 147.3 (C-4), 143.5 (C-9), 129.2 (C-3), 128.7 (C-10), 128.2 (C-12), 127.2 (C-11), 123.2 (C-2), $68.4(\mathrm{C}-5), 62.6$ (C-7), 14.1 (C-8).

IR (neat film, $\mathrm{cm}^{-1}$ ): 3106, 2923, 2853, 2360, 2343, 1737, 1530, 1368, 1348, 1307, 1193, 1146, 1080, 1014, 854, 750, 734, 696, 683, 612.

MS $\left(\mathrm{ES}^{+}\right)$found $m / z$ 301 $[\mathrm{M}+\mathrm{H}]^{+}$; HRMS $\left(\mathrm{ES}^{+}\right.$) found 323.0998, $\mathrm{C}_{16} \mathrm{H}_{16} \mathrm{~N}_{2} \mathrm{O}_{4}[\mathrm{M}+\mathrm{Na}]^{+}$ requires 323.1002.

1-(Methylamino)-1-(4-nitrophenyl)-1-phenylpropan-2-one 8c

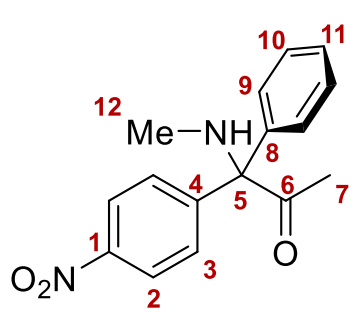

Prepared according to General Procedure E from 4-nitro- $N$ methylbenzenesulfonamide $6 \mathbf{a}$ (0.10 mmol, $21.6 \mathrm{mg}), \mathrm{K}_{2} \mathrm{CO}_{3}(0.30$ mmol, $41.5 \mathrm{mg}$ ), 1-chloro-1-phenylpropan-2-one (o.20 mmol, 30 $\mu \mathrm{L})$, DMF (1.0o $\mathrm{mL})$. Purification by column chromatography on silica gel (o-40\% EtOAc in hexanes) afforded 8c as a yellow oil (10.2 mg, 0.036 mmol, 36\%).

${ }^{1} \mathrm{H}$ NMR (40o MHz, $\mathrm{CDCl}_{3}$ ): $\delta 8.19$ (app. d, $J=9.0 \mathrm{~Hz}, 2 \mathrm{H}, \mathrm{CH}_{\mathrm{ar}}-2$ ), 7.64 (app. d, $J=9.0$ $\left.\mathrm{Hz}, 2 \mathrm{H}, \mathrm{CH}_{\mathrm{ar}}-3\right), 7 \cdot 39-7 \cdot 30\left(\mathrm{~m},{ }_{5} \mathrm{H}, \mathrm{CH}_{\mathrm{ar}}-9,10,11\right), 2.15\left(\mathrm{~s}, 3 \mathrm{H}, \mathrm{CH}_{3}-7\right), 2.11\left(\mathrm{~s},{ }_{3} \mathrm{H}, \mathrm{CH}_{3}-12\right)$; ${ }^{13} \mathrm{C}$ NMR (101 MHz, CDCl $): \delta 205.8$ (C-6), 147.6 (C-1), 147.1 (C-4), 139.4 (C-8), $130.2(\mathrm{C}-$ 3), 128.9 (C-10), 128.3 (C-9), 128.2 (C-11), 123.4 (C-2), 77.7 (C-5), 30.3 (C-12), 26.3 (C-7).

IR (neat film, $\mathrm{cm}^{-1}$ ): 3353, 3063, 2944, 2804, 1709, 1593, 1517, 1490, 1448, 1346, 1172, 1141, 1110, 850, 764, 747, 701, 668, 613 .

MS $\left(\mathrm{ES}^{+}\right.$) found $\mathrm{m} / \mathrm{z} 301[\mathrm{M}+\mathrm{Na}]^{+}$; HRMS $\left(\mathrm{ES}^{+}\right)$found 285.1220, $\mathrm{C}_{16} \mathrm{H}_{17} \mathrm{~N}_{2} \mathrm{O}_{3}[\mathrm{M}+\mathrm{H}]^{+}$ requires 285.1234 .

Ethyl 2-(methylamino)-2-(2-nitrophenyl)-2-phenylacetate 8d

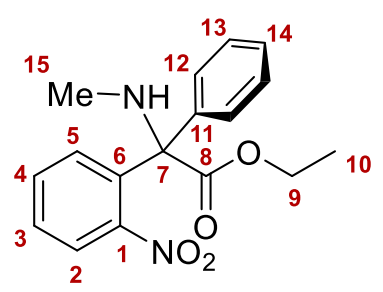

Prepared according to General Procedure E from 2-nitro- $N$ methylbenzenesulfonamide $\mathbf{6 b}$ ( $0.10 \mathrm{mmol}, 21.6 \mathrm{mg}), \mathrm{K}_{2} \mathrm{CO}_{3}$ (0.30 mmol, $41.5 \mathrm{mg}$ ), ethyl $\alpha$-chlorophenylacetate $7 \mathrm{a}$ (0.20 mmol, $34 \mu \mathrm{L})$, DMF (1.00 $\mathrm{mL})$. Purification by column chromatography on silica gel (o-40\% EtOAc in hexanes) 
afforded $8 \mathrm{~d}$ as a yellow solid (15.5 mg, $0.049 \mathrm{mmol}, 49 \%)$, m.p. $103-105{ }^{\circ} \mathrm{C}$.

${ }^{1} \mathrm{H}$ NMR (400 MHz, $\mathrm{CDCl}_{3}$ ): $\delta 7.92$ (app. d, $\left.J=8.0 \mathrm{~Hz},{ }_{1} \mathrm{H}, \mathrm{CH}_{\mathrm{ar}}-2\right), 7.62-7.58(\mathrm{~m}, 2 \mathrm{H}$,

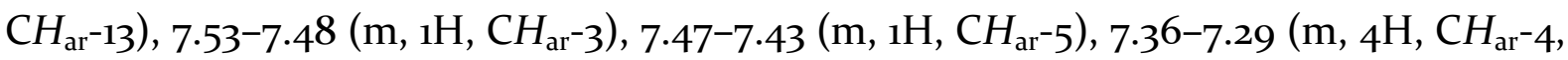
12, 14), 4.19-4.02 (m, 2H, $\mathrm{CH}_{2}-9$ ), 2.72 (br. s, $1 \mathrm{H}, \mathrm{NH}$ ), 2.19 (s, $3 \mathrm{H}, \mathrm{CH}_{3}-15$ ), 1.13 (t, J = 7.2 $\left.\mathrm{Hz}, 3 \mathrm{H}, \mathrm{CH}_{3}-10\right) ;{ }^{13} \mathrm{C}$ NMR (101 $\mathrm{MHz}, \mathrm{CDCl}_{3}$ ): $\delta 171.1$ (C-8), 150.0 (C-1), 138.9 (C-11), 135.8 (C-6), 132.8 (C-4), 131.8 (C-3), 129.0 (C-13), 128.4 (C-5), 128.2 (C-12), 128.1 (C-14), 125.4 (C2), $72.2(\mathrm{C}-7), 61.9$ (C-9), 30.9 (C-15), 13.9 (C-10).

IR (neat film, cm $\mathrm{cm}^{-1}$ ): 3324, 3075, 2951, 2806, 1819, 1724, 1524, 1445, 1350, 1236, 1206, 1176, 1163, 1100, 1022, 968, 851, 799, 772, 744, 738, 709, 699, 692, 635.

MS $\left(\mathrm{ES}^{+}\right)$found $m / z 315[\mathrm{M}+\mathrm{H}]^{+}$; HRMS $\left(\mathrm{ES}^{+}\right.$) found 315.1339, $\mathrm{C}_{17} \mathrm{H}_{19} \mathrm{~N}_{2} \mathrm{O}_{4}[\mathrm{M}+\mathrm{H}]^{+}$ requires 315.1347 .

Ethyl 2-(methylamino)-2-(4-nitro-3-(trifluoromethyl)phenyl)-2-phenylacetate $8 \mathbf{e}$

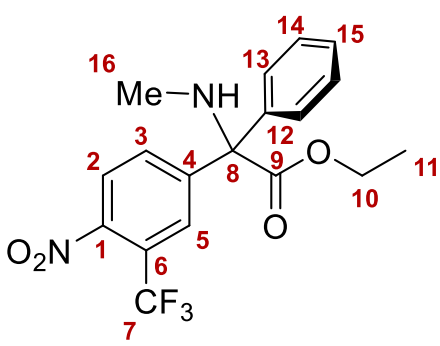

Prepared according to General Procedure E from 4-nitro3-(trifluoromethyl)- $N$-methylbenzenesulfonamide 6c (o.11 mmol, $31.6 \mathrm{mg}$ ), $\mathrm{K}_{2} \mathrm{CO}_{3}$ (o.33 mmol, $45.6 \mathrm{mg}$ ), ethyl $\alpha-$ chlorophenylacetate $7 \mathbf{a}$ (o.22 mmol, $38 \mu \mathrm{L})$, DMF (1.00 mL).

Purification by column chromatography on silica gel (o40\% EtOAc in hexanes) afforded $\mathbf{1 5 0 g}$ as a clear yellow oil (17.9 mg, $0.047 \mathrm{mmol}, 42 \%)$.

${ }^{1} \mathrm{H}$ NMR (400 MHz, $\mathrm{CDCl}_{3}$ ): $\delta 8.14$ (app. d, $J=1.8 \mathrm{~Hz},{ }_{1} \mathrm{H}, \mathrm{CH}_{\mathrm{ar}}{ }^{-5}$ ), 7.97 (app. dd, $J=8.6$, $\left.1.8 \mathrm{~Hz}, 1 \mathrm{H}, \mathrm{CH}_{\mathrm{ar}}-3\right), 7.82$ (app. d, $\left.J=8.6 \mathrm{~Hz}, 1 \mathrm{H}, \mathrm{CH}_{\mathrm{ar}}-2\right), 7.38-7.28\left(\mathrm{~m}, 5 \mathrm{H}, \mathrm{CH}_{\mathrm{ar}}-13,14\right.$, 15), 4.34-4.23 (m, $2 \mathrm{H}, \mathrm{CH}_{2}-10$ ), 2.46 (br. s, $1 \mathrm{H}, \mathrm{NH}$ ), 2.18 (s, $\left.3 \mathrm{H}, \mathrm{CH}_{3}-16\right), 1.24$ (t, J = 7.1 $\left.\mathrm{Hz}, 3 \mathrm{H}, \mathrm{CH}_{3}-11\right) ;{ }^{13} \mathrm{C}$ NMR (101 MHz, $\mathrm{CDCl}_{3}$ ): $\delta 172.1$ (C-9), 146.9 (C-4), 140.5 (C-12), 138.5 (C-1), 133.7 (C-3), 128.8 (C-13), 128.5 (C-5), 128.3 (C-15), 127.7 (q, J = 206.5 Hz, C-7), 127.4 (C-14), $124.7(\mathrm{C}-2), 123.3$ (q, J = 33.8, C-6), $73.0(\mathrm{C}-8), 62.4(\mathrm{C}-10), 30.9(\mathrm{C}-16), 14.2(\mathrm{C}-11)$; ${ }^{19}$ F NMR $\left(376 \mathrm{MHz}, \mathrm{CDCl}_{3}\right): \delta-59.83$.

IR (neat film, $\mathrm{cm}^{-1}$ ): 3356, 3066, 2983, 2929, 2807, 2362, 1729, 1539, 1360, 1311, 1215, 1179, $1141,1048,1027,858,758,701,657$. 
MS $\left(\mathrm{ES}^{+}\right)$found $\mathrm{m} / \mathrm{z} 383[\mathrm{M}+\mathrm{H}]^{+}$; HRMS $\left(\mathrm{ES}^{+}\right.$) found $383.1205, \mathrm{C}_{18} \mathrm{H}_{18} \mathrm{~F}_{3} \mathrm{~N}_{2} \mathrm{O}_{4}[\mathrm{M}+\mathrm{H}]^{+}$ requires 383.1213 .

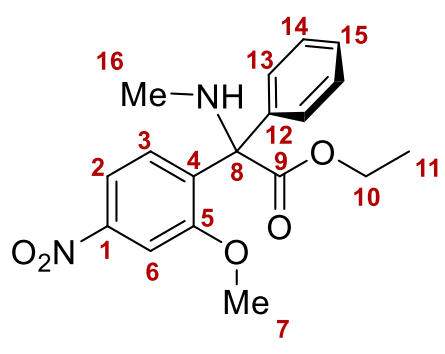

Ethyl 2-(2-methoxy-4-nitrophenyl)-2-(methylamino)-2phenylacetate $8 f$

Prepared according to General Procedure E from 4-nitro2-methoxy- $N$-methylbenzenesulfonamide 6d (o.11 mmol, $26.4 \mathrm{mg}), \quad \mathrm{K}_{2} \mathrm{CO}_{3}(0.32 \mathrm{mmol}, 44.2 \mathrm{mg})$, ethyl $\alpha-$ chlorophenylacetate 152a (0.21 mmol, $36 \mu \mathrm{L})$, DMF (1.00 $\mathrm{mL})$. Purification by column chromatography on silica gel (o-40\% EtOAc in hexanes) afforded $8 f$ as a clear yellow oil (17.7 mg, 0.059 mmol, 56\%).

${ }^{1} \mathrm{H}$ NMR (400 $\mathrm{MHz}, \mathrm{CDCl}_{3}$ ): $\delta 7.79$ (app. dd, $\left.J=8.6,2.2 \mathrm{~Hz}, 1 \mathrm{H}, \mathrm{CH}_{\mathrm{ar}}-2\right)$ ) 7.73-7.68 (m, $\left.3 \mathrm{H}, \mathrm{CH}_{\mathrm{ar}}-6,14\right), 7.47$ (app. d, $\left.J=8.6 \mathrm{~Hz}, 1 \mathrm{H}, \mathrm{CH}_{\mathrm{ar}}-3\right)$ ) 7.38-7.32 (m, $2 \mathrm{H}, \mathrm{CH}_{\mathrm{ar}}-13$ ), 7.32$7.27\left(\mathrm{~m},{ }_{1} \mathrm{H}, \mathrm{CH}_{\mathrm{ar}}-15\right), 4.23-4.12\left(\mathrm{~m}, 2 \mathrm{H}, \mathrm{CH}_{2}-10\right)$, 3.91 (s, $\left.3 \mathrm{H}, \mathrm{CH}_{3}-7\right), 2.12\left(\mathrm{~s}, 3 \mathrm{H}, \mathrm{CH}_{3}-16\right)$, $1.17\left(\mathrm{t}, 3 \mathrm{H}, J=7.2 \mathrm{~Hz}, \mathrm{CH}_{3}-11\right) ;{ }^{13} \mathrm{C}$ NMR (101 MHz, $\left.\mathrm{CDCl}_{3}\right): \delta 172.2(\mathrm{C}-9), 157.2(\mathrm{C}-5), 148.1$ (C-1), 138.7 (C-4), 137.6 (C-12), 129.5 (C-3), 129.0 (C-14), 128.1 (C-13), 127.9 (C-15), 115.6 (C2), 106.1 (C-6), 69.9 (C-8), 61.4 (C-10), 56.1 (C-7), 30.6 (C-16), 14.3 (C-11).

IR (neat film, $\mathrm{cm}^{-1}$ ): 3379, 3331, 3090, 3059, 2979, 2943, 2853, 2803, 1733, 1590, 1520, 1485, 1344, 1251, 1179, 1094, 1028, 867, 767, 739, 700.

MS $\left(\mathrm{ES}^{+}\right.$) found $m / z 345[\mathrm{M}+\mathrm{H}]^{+}$; HRMS $\left(\mathrm{ES}^{+}\right.$) found 345.1445, $\mathrm{C}_{18} \mathrm{H}_{21} \mathrm{~N}_{2} \mathrm{O}_{5}[\mathrm{M}+\mathrm{H}]^{+}$ requires 345.1431

\section{Ethyl 2-(4-nitrophenyl)-2-phenyl-2-(phenylamino) acetate $8 \mathrm{~g}$}

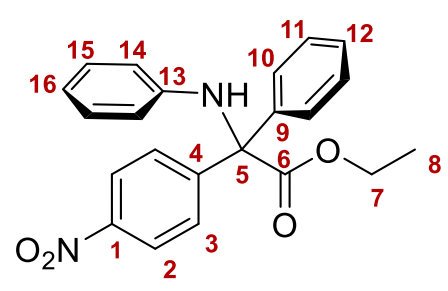

Prepared according to General Procedure E from 4-nitro$N$-phenylbenzenesulfonamide $6 \mathbf{e}(0.10 \mathrm{mmol}, 27.8 \mathrm{mg})$, $\mathrm{K}_{2} \mathrm{CO}_{3}$ (0.30 mmol, $41.5 \mathrm{mg}$ ), ethyl $\alpha$-chlorophenylacetate $7 \mathbf{a}$ (o.20 mmol, $34 \mu \mathrm{L}$ ), DMF (1.0o mL). Purification by column chromatography on silica gel (o-40\% EtOAc in hexanes) afforded $8 \mathrm{~g}$ as a yellow oil (32.3 $\mathrm{mg}$, $0.086 \mathrm{mmol}, 86 \%)$. 
${ }^{1}$ H NMR (400 MHz, $\mathrm{CDCl}_{3}$ ): $\delta 8.15$ (app. d, $J=8.4 \mathrm{~Hz}, 2 \mathrm{H}, \mathrm{CH}_{\mathrm{ar}}-2$ ), 7.84 (app. d, $J=8.4$ $\left.\mathrm{Hz}, 2 \mathrm{H}, \mathrm{CH}_{\mathrm{ar}}-3\right)$ ) 7.49-7.46 (m, 2H, $\left.\mathrm{CH}_{\mathrm{ar}}-11\right)$, 7.38-7.28 (m, 3H, $\left.\mathrm{CH}_{\mathrm{ar}}-10,12\right)$, 7.03 (app. dd, $\left.J=8.6,7.5 \mathrm{~Hz}, 2 \mathrm{H}, \mathrm{CH}_{\mathrm{ar}}-15\right), 6.71$ (app. tt, $J=7.5,1.1 \mathrm{~Hz}, 1 \mathrm{H}, \mathrm{CH}_{\mathrm{ar}}-16$ ), 6.44 (app. dd, $J$ = 8.6, $\left.1.1 \mathrm{~Hz}, 2 \mathrm{H}, \mathrm{CH}_{\mathrm{ar}}-14\right), 5.33(\mathrm{~s}, 1 \mathrm{H}, \mathrm{NH}), 4.26-4.12\left(\mathrm{~m}, 2 \mathrm{H}, \mathrm{CH}_{2}-7\right), 1.03(\mathrm{t}, J=7.1 \mathrm{~Hz}$, $\left.3 \mathrm{H}, \mathrm{CH}_{3}-8\right) ;{ }^{13} \mathrm{C}$ NMR (101 MHz, $\left.\mathrm{CDCl}_{3}\right): \delta 172.1(\mathrm{C}-6), 147.4(\mathrm{C}-4), 147.2(\mathrm{C}-1)$, 145.o (C13), 140.5 (C-9), 129.9 (C-3), 129.0 (C-10), 128.9 (C-15), 128.5 (C-12), 127.5 (C-11), 123.2 (C2), 119.1 (C-16), 115.7 (C-14), 71.7 (C-5), 62.7 (C-7), 13.8 (C-8).

IR (neat film, $\mathrm{cm}^{-1}$ ): 3392, 3054, 2981, 1731, 1602, 1518, 1498, 1347, 1257, 1224, 1196, 1023, 1015, 854, 750, 736, 720, 694 .

MS $\left(\mathrm{ES}^{+}\right.$) found $m / z$ z99 $[\mathrm{M}+\mathrm{Na}]^{+}$; HRMS $\left(\mathrm{ES}^{+}\right.$) found 399.1315, $\mathrm{C}_{22} \mathrm{H}_{20} \mathrm{~N}_{2} \mathrm{O}_{4} \mathrm{Na}[\mathrm{M}+\mathrm{Na}]^{+}$ requires 399.1305 .

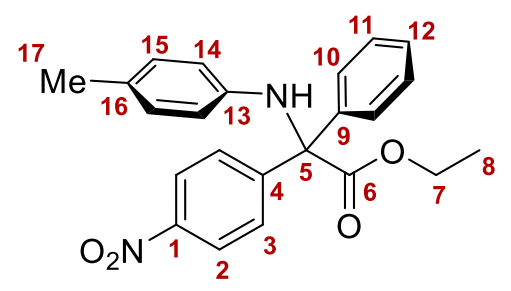

Ethyl

2-(4-nitrophenyl)-2-phenyl-2-(p-

\section{tolylamino)acetate $8 \mathrm{~h}$}

Prepared according to General Procedure E from 4nitro- $N$-( $p$-tolyl)benzenesulfonamide 6 f (o.10 mmol, 29.2 $\mathrm{mg}), \quad \mathrm{K}_{2} \mathrm{CO}_{3} \quad(0.30 \mathrm{mmol}, 41.5 \mathrm{mg})$, ethyl $\alpha-$ chlorophenylacetate $7 \mathbf{a}(0.20 \mathrm{mmol}, 34 \mu \mathrm{L})$, DMF (1.0o $\mathrm{mL}$ ). Purification by column chromatography on silica gel (o-40\% EtOAc in hexanes) afforded $8 \mathrm{~h}$ as a yellow oil (36 $\mathrm{mg}$, $0.092 \mathrm{mmol}, 92 \%)$.

${ }^{1} \mathrm{H}$ NMR (400 MHz, $\mathrm{CDCl}_{3}$ ): $\delta 8.14$ (app. d, $J=9.0 \mathrm{~Hz}, 2 \mathrm{H}, \mathrm{CH}_{\mathrm{ar}}-2$ ), 7.84 (app. d, $J=9.0$ $\mathrm{Hz}, 2 \mathrm{H}, \mathrm{CH}_{\mathrm{ar}-3}$ ), 7.49-7.46 (m, 2H, $\left.\mathrm{CH}_{\mathrm{ar}}-11\right)$, 7.37-7.28 (m, $\left.3 \mathrm{H}, \mathrm{CH}_{\mathrm{ar}}-10,12\right), 6.84$ (app. d, $J=8.3 \mathrm{~Hz}, 2 \mathrm{H}, \mathrm{CH}_{\mathrm{ar}}{ }^{-15}$ ), 6.35 (app. d, $J=8.3 \mathrm{~Hz}, 2 \mathrm{H}, \mathrm{CH}_{\mathrm{ar}}-14$ ), 5.21 (s, $\left.1 \mathrm{H}, \mathrm{NH}\right), 4.25-4.13$ (m, $\left.2 \mathrm{H}, \mathrm{CH}_{2}-7\right), 2.17\left(\mathrm{~s}, 3 \mathrm{H}, \mathrm{CH}_{3}-17\right), 1.05\left(\mathrm{t}, J=7.2 \mathrm{~Hz}, 3 \mathrm{H}, \mathrm{CH}_{3}-8\right) ;{ }^{13} \mathrm{C}$ NMR (101 MHz, $\left.\mathrm{CDCl}_{3}\right): \delta 172.2$ (C-6), 147.6 (C-1), 147.1 (C-4), 142.5 (C-16), 140.7 (C-13), 129.9 (C- 3), 129.5 (C-15), 128.8 (C-10), 128.4 (C-12), 128.3 (C-9), 127.5 (C-11), 123.2 (C-2), 115.8 (C-14), 71.7 (C5), $62.7(\mathrm{C}-7), 20.5(\mathrm{C}-17), 13.9(\mathrm{C}-8)$.

IR (neat film, cm ${ }^{-1}$ ): 3390, 2980, 2920, 2865, 2359, 1731, 1515, 1348, 1301, 1254, 1226, 1195, $1024,1015,855,812,736,703$. 
MS $\left(\mathrm{ES}^{+}\right.$) found $m / z 413[\mathrm{M}+\mathrm{Na}]^{+}$; HRMS $\left(\mathrm{ES}^{+}\right)$found 413.1469, $\mathrm{C}_{23} \mathrm{H}_{22} \mathrm{~N}_{2} \mathrm{O}_{4} \mathrm{Na}[\mathrm{M}+\mathrm{Na}]^{+}$ requires 413.1472 .

Ethyl 2-(mesitylamino)-2-(4-nitrophenyl)-2-phenylacetate 8i

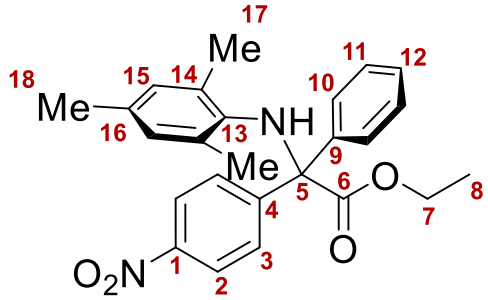

Prepared according to General Procedure E from 4nitro- $N$-mesitylbenzenesulfonamide $\mathbf{6 g}$ (0.10 $\mathrm{mmol}, 32.0$ $\mathrm{mg}), \quad \mathrm{K}_{2} \mathrm{CO}_{3} \quad(0.30 \mathrm{mmol}, 41.5 \mathrm{mg})$, ethyl $\alpha-$ chlorophenylacetate $7 \mathrm{a}(0.20 \mathrm{mmol}, 34 \mu \mathrm{L})$, DMF (1.0o $\mathrm{mL})$. Purification by column chromatography on silica gel (o-40\% EtOAc in hexanes) afforded $8 \mathbf{i}$ as a light red solid (9.7 mg, $0.023 \mathrm{mmol}, 23 \%$ ),

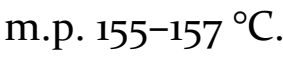

${ }^{1} \mathrm{H}$ NMR (40o MHz, $\mathrm{CDCl}_{3}$ ): $\delta 8.27$ (app. d, J=8.9 Hz, 2H, $\mathrm{CH}_{\mathrm{ar}}-2$ ), 7.85 (app. d, $J=8.9$ $\mathrm{Hz}, 2 \mathrm{H}, \mathrm{CH}_{\mathrm{ar}-3}$ ), 7.21-7.15 (m, $\left.3 \mathrm{H}, \mathrm{CH}_{\mathrm{ar}}-10,12\right)$, 7.11-7.07 (m, 2H, $\left.\mathrm{CH}_{\mathrm{ar}}-11\right), 6.72$ (br. s, $1 \mathrm{H}$, $\left.\mathrm{CH}_{\mathrm{ar}}-15\right), 6.55$ (br. s, $1 \mathrm{H}, \mathrm{CH}_{\mathrm{ar}^{-15}}$ ), $5.74(\mathrm{~s}, 1 \mathrm{H}, \mathrm{NH}), 4.19$ (m, $\left.2 \mathrm{H}, \mathrm{CH}-7\right), 2.15$ (s, ${ }_{3} \mathrm{H}, \mathrm{CH}-$ 17), $2.13\left(\mathrm{~s}, 3 \mathrm{H}, \mathrm{CH}_{3}-17\right), 1.79\left(\mathrm{~s}, 3 \mathrm{H}, \mathrm{CH}_{3}-18\right), 1.22\left(\mathrm{t}, J=7.1 \mathrm{~Hz}, 3 \mathrm{H}, \mathrm{CH}_{3}-8\right) ;{ }^{13} \mathrm{C}$ NMR (101 $\left.\mathrm{MHz}, \mathrm{CDCl}_{3}\right): \delta 170.6(\mathrm{C}-6), 150.1(\mathrm{C}-4), 145.7$ (C-1), 140.7 (C-9), 139.6 (C-16), 138.8 (C13), 131.9 (C-14), 131.3 (C-14), 130.2 (C-10), 129.94 (C-3), 129.86 (C-12), 129.7 (C-15), 129.3 (C-15), 128.2 (C-11), 123.8 (C-2), 68.5 (C-5), 62.0 (C-7), 21.0 (C-17), 20.0 (C-18), 19.1 (C-17), 14.1 (C-8).

IR (neat film, $\mathrm{cm}^{-1}$ ): 3105, 3066, 2982, 2926, 2867, 1743, 1530, 1378, 1311, 1203, 1165, 1090, $1020,853,738,699,689,625$.

MS $\left(\mathrm{ES}^{+}\right)$found $m / z$ 441 $[\mathrm{M}+\mathrm{Na}]^{+}$; HRMS $\left(\mathrm{ES}^{+}\right)$found 441.1783, $\mathrm{C}_{25} \mathrm{H}_{26} \mathrm{~N}_{2} \mathrm{O}_{4} \mathrm{Na}[\mathrm{M}+\mathrm{Na}]^{+}$ requires 441.1785 .

\section{Ethyl 2-((4-methoxyphenyl)amino)-2-(4-nitrophenyl)-2-phenylacetate 8j}

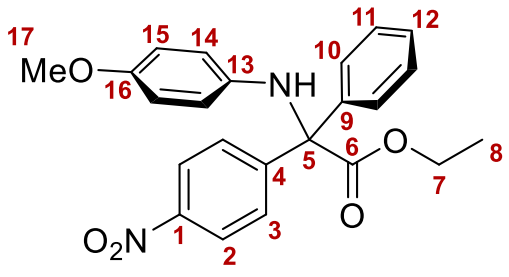

Prepared according to General Procedure E from 4nitro- $N$-(4-methoxyphenyl)benzenesulfonamide $\mathbf{6 h}$ (o.10 mmol, $30.8 \mathrm{mg}$ ), $\mathrm{K}_{2} \mathrm{CO}_{3}$ (0.30 mmol, $41.5 \mathrm{mg}$ ), ethyl $\alpha-$ chlorophenylacetate 7a (o.20 mmol, $34 \mu \mathrm{L})$, DMF (1.0o $\mathrm{mL})$. Purification by column chromatography on silica 
gel (o-40\% EtOAc in hexanes) afforded 8j as a clear yellow oil (38.7 mg, $0.095 \mathrm{mmol}$, $95 \%)$.

${ }^{1} \mathrm{H}$ NMR (400 MHz, $\mathrm{CDCl}_{3}$ ): $\delta 8.14$ (app. d, $J=9.2 \mathrm{~Hz}, 2 \mathrm{H}, \mathrm{CH}_{\mathrm{ar}}-2$ ), 7.83 (app. d, $J=9.2$ $\left.\mathrm{Hz}, 2 \mathrm{H}, \mathrm{CH}_{\mathrm{ar}}-3\right)$, 7.48-7.44 (m, 2H, $\left.\mathrm{CH}_{\mathrm{ar}}-11\right), 7.36-7.27$ (m, 3H, $\left.\mathrm{CH}_{\mathrm{ar}}-10,12\right), 6.61$ (app. d, $\left.J=8.4 \mathrm{~Hz}, 2 \mathrm{H}, \mathrm{CH}_{\mathrm{ar}}-15\right), 6.39$ (app. d, $J=8.4 \mathrm{~Hz}, 2 \mathrm{H}, \mathrm{CH}_{\mathrm{ar}}-14$ ), 5.04 (br. s, $1 \mathrm{H}, \mathrm{NH}$ ), 4.25-4.11 (m, 2H, $\left.\mathrm{CH}_{2}-7\right), 3.67\left(\mathrm{~s}, 3 \mathrm{H}, \mathrm{CH}_{3}-17\right), 1.04\left(\mathrm{t}, J=7.2 \mathrm{~Hz}, 3 \mathrm{H}, \mathrm{CH}_{3}-8\right) ;{ }^{13} \mathrm{C} \mathrm{NMR}$ (101 MHz, $\mathrm{CDCl}_{3}$ ): $\delta 172.3$ (C-6), 153.1 (C-16), 147.9 (C-1), 147.1 (C-4), 140.9 (C-9), 138.8 (C-13), 129.8 (C-3), 128.8 (C-10), 128.4 (C-12), 127.4 (C-11), 123.2 (C-2), 117.3 (C-14), 114.4 (C-15), $72.1(\mathrm{C}-5), 62.6$ (C-7), 55.6 (C-17), 13.9 (C-8).

IR (neat film, $\mathrm{cm}^{-1}$ ): 3378, 3062, 2979, 2933, 2833, 1731, 1510, 1347, 1237, 1195, 1179, 1026, $1015,854,823,763,735,701$.

MS $\left(\mathrm{ES}^{+}\right.$) found $m / z 429[\mathrm{M}+\mathrm{Na}]^{+}$; HRMS $\left(\mathrm{ES}^{+}\right.$) found 407.1593, $\mathrm{C}_{23} \mathrm{H}_{23} \mathrm{~N}_{2} \mathrm{O}_{5}[\mathrm{M}+\mathrm{H}]^{+}$ requires 407.1601 .

\section{Ethyl 2-((4-chlorophenyl)amino)-2-(4-nitrophenyl)-2-phenylacetate 8k}

Prepared according to General Procedure E from 4-nitro- $\mathrm{N}-\left(4^{-}\right.$

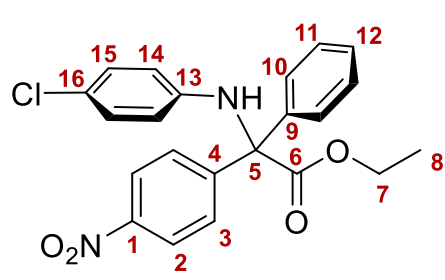
chlorophenyl)benzenesulfonamide $6 \mathbf{i}$ ( $0.10 \mathrm{mmol}, 31.3 \mathrm{mg}$ ), $\mathrm{K}_{2} \mathrm{CO}_{3}(0.30 \mathrm{mmol}, 41.5 \mathrm{mg})$, ethyl $\alpha$-chlorophenylacetate $7 \mathbf{a}$ (o.20 mmol, $34 \mu \mathrm{L}$ ), DMF (1.00 mL). Purification by column chromatography on silica gel (o-40\% EtOAc in hexanes) afforded 8k as a vibrant yellow solid (32.3 mg, 0.076 mmol, $76 \%$ ), m.p. $48-51^{\circ} \mathrm{C}$.

${ }^{1} \mathrm{H}$ NMR (500 MHz, $\mathrm{CDCl}_{3}$ ): $\delta 8.08$ (app. d, $J=9.0 \mathrm{~Hz}, 2 \mathrm{H}, \mathrm{CH}_{\mathrm{ar}}-2$ ), 7.72 (app. d, $J=9.0$ $\left.\mathrm{Hz}, 2 \mathrm{H}, \mathrm{CH}_{\mathrm{ar}}-3\right), 7 \cdot 38-7.35\left(\mathrm{~m}, 2 \mathrm{H}, \mathrm{CH}_{\mathrm{ar}}-10\right)$, 7.29-7.25 (m, $\left.2 \mathrm{H}, \mathrm{CH}_{\mathrm{ar}}-11\right)$, 7.25-7.21 (m, ${ }_{1} \mathrm{H}, \mathrm{CH}_{\mathrm{ar}}-12$ ), 6.89 (app. dt, $\left.J=8.8,3.4 \mathrm{~Hz}, 2 \mathrm{H}, \mathrm{CH}_{\mathrm{ar}}-15\right), 6.27$ (app. dt, $J=8.8,3.4 \mathrm{~Hz}$, $\left.2 \mathrm{H}, \mathrm{CH}_{\mathrm{ar}}-14\right), 5.35(\mathrm{~s}, 1 \mathrm{H}, \mathrm{NH}), 4.18-4.08\left(\mathrm{~m}, 2 \mathrm{H}, \mathrm{CH}_{2}-7\right), 0.99\left(\mathrm{t}, J=7.2 \mathrm{~Hz}, 3 \mathrm{H}, \mathrm{CH}_{3}-8\right)$; ${ }^{13} \mathrm{C}$ NMR (126 MHz, CDCl $): \delta 171.9$ (C-6), 147.3 (C-1), 146.7 (C-4), $143.4(\mathrm{C}-13), 139.7$ (C9), 129.9 (C-3), 128.9 (C-11), 128.8 (C-15), 128.6 (C-16), 127.7 (C-10), 123.9 (C-12), 123.3 (C2), 116.8 (C-14), 71.6 (C-5), 63.0 (C-7), $13.9(\mathrm{C}-8)$. 
IR (neat film, $\mathrm{cm}^{-1}$ ): 3389, 2981, 1732, 1595, 1525, 1498, 1447, 1347, 1314, 1294, 1255, 1226, $1015,819,735,702$.

MS $\left(\mathrm{ES}^{+}\right)$found $m / z 449[\mathrm{M}+\mathrm{K}]^{+} ;$HRMS $\left(\mathrm{ES}^{+}\right.$) found 449.0665, $\mathrm{C}_{22} \mathrm{H}_{19} \mathrm{ClN}_{2} \mathrm{O}_{4} \mathrm{~K}[\mathrm{M}+\mathrm{K}]^{+}$ requires 449.0655 .

Ethyl 4-((2-ethoxy-1-(4-nitrophenyl)-2-oxo-1-phenylethyl)amino)benzoate 81

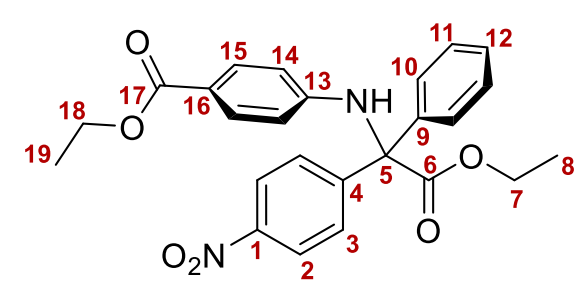

Prepared according to General Procedure E from ethyl 4-((4-nitrophenyl)sulfonamido)benzoate $\quad \mathbf{6 j}$ (o.10 mmol, 35.0 mg), $\mathrm{K}_{2} \mathrm{CO}_{3}$ (0.30 mmol, $41.5 \mathrm{mg}$ ), ethyl $\alpha$-chlorophenylacetate $7 \mathbf{a}$ (0.20 mmol, $34 \mu \mathrm{L})$, DMF (1.00 $\mathrm{mL})$. Purification by column chromatography on silica gel (o-40\% EtOAc in hexanes) afforded $\mathbf{8 1}$ as a light yellow oil (11.3 mg, $0.025 \mathrm{mmol}, 25 \%)$.

${ }^{1} \mathrm{H}$ NMR (40o MHz, $\mathrm{CDCl}_{3}$ ): $\delta 8.17$ (app. d, $J=9.0 \mathrm{~Hz}, 2 \mathrm{H}, \mathrm{CH}_{\mathrm{ar}}-2$ ), 7.79 (app. d, $J=9.0$ $\left.\mathrm{Hz}, 2 \mathrm{H}, \mathrm{CH}_{\mathrm{ar}}-3\right), 7.72$ (app. d, $\left.J=8.8 \mathrm{~Hz}, 2 \mathrm{H}, \mathrm{CH}_{\mathrm{ar}}-14\right), 7.47-7.41\left(\mathrm{~m}, 2 \mathrm{H}, \mathrm{CH}_{\mathrm{ar}}-11\right), 7.39-$ $7.32\left(\mathrm{~m}, 3 \mathrm{H}, \mathrm{CH}_{\mathrm{ar}-10}, 12\right), 6.40$ (app. d, $J=8.8 \mathrm{~Hz}, 2 \mathrm{H}, \mathrm{CH}_{\mathrm{ar}^{-15}}$ ), 5.91 (br. s, $1 \mathrm{H}, \mathrm{NH}$ ), 4.29-4.18 (m, $\left.4 \mathrm{H}, \mathrm{CH}_{2}-7,18\right), 1.31$ (t, $\left.J=7.1 \mathrm{~Hz},{ }_{3} \mathrm{H}, \mathrm{CH}_{3}-19\right), 1.08\left(\mathrm{t}, J=7.2 \mathrm{~Hz}, 3 \mathrm{H}, \mathrm{CH}_{3}-\right.$ 8); ${ }^{13} \mathrm{C}$ NMR (101 MHz, CDCl $)$ ): $\delta 171.8$ (C-6), 166.6 (C-17), 148.6 (C-16), $147.4(\mathrm{C}-4), 146.1$ (C-1), 138.8 (C-9), 131.0 (C-14), 130.0 (C-3), 129.0 (C-10), 128.8 (C-12), 127.9 (C-11), 123.4 (C-2), 120.6 (C-13), 114.6 (C-15), 71.2 (C-5), 63.3 (C-18), 60.5 (C-7), 14.6 (C-19), 13.8 (C-8).

IR (neat film, cm ${ }^{-1}$ ): 3379, 2981, 2930, 2856, 1733, 1703, 1601, 1519, 1348, 1266, 1229, 1178, 1108, 771, 701 .

MS $\left(\mathrm{ES}^{-}\right)$found $m / z 447[\mathrm{M}-\mathrm{H}]^{-}$; HRMS $\left(\mathrm{ES}^{-}\right)$found 447.1565, $\mathrm{C}_{25} \mathrm{H}_{23} \mathrm{~N}_{2} \mathrm{O}_{6}[\mathrm{M}-\mathrm{H}]^{-}$ requires 447.1562 .

Ethyl 2-(benzylamino)-2-(4-nitrophenyl)-2-phenylacetate 8m 
Prepared according to General Procedure E from 4-nitro- $N$ benzylbenzenesulfonamide $6 \mathrm{~m}$ (0.10 mmol, $29.2 \mathrm{mg}), \mathrm{K}_{2} \mathrm{CO}_{3}(0.30 \mathrm{mmol}, 41.5 \mathrm{mg})$,

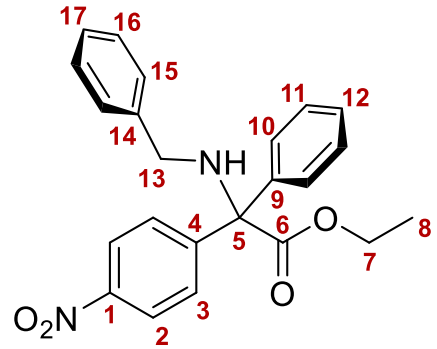
ethyl $\alpha$-chlorophenylacetate $7 \mathbf{a}$ (o.20 mmol, $34 \mu \mathrm{L})$, DMF (1.0o $\mathrm{mL})$. Purification by column chromatography on silica gel (o-40\% EtOAc in hexanes) afforded $\mathbf{8 m}$ as a clear, colourless oil (86.7 mg, $0.22 \mathrm{mmol}, 74 \%)$.

${ }^{1}$ H NMR (400 $\mathrm{MHz}, \mathrm{CDCl}_{3}$ ): $\delta 8.07$ (app. d, $J=9.1 \mathrm{~Hz}, 2 \mathrm{H}$, $\left.\mathrm{CH}_{\mathrm{ar}}-2\right), 7.74$ (app. d, $\left.J=9.1 \mathrm{~Hz}, 2 \mathrm{H}, \mathrm{CH}_{\mathrm{ar}}-3\right), 7 \cdot 39-7.35(\mathrm{~m}$, $\left.2 \mathrm{H}, \mathrm{CH}_{\mathrm{ar}}-10\right)$, 7.29-7.21 (m, 6H, $\left.\mathrm{CH}_{\mathrm{ar}-11,15}, 16\right), 7.20-7.15\left(\mathrm{~m}, 2 \mathrm{H}, \mathrm{CH}_{\mathrm{ar}}-12,17\right), 4.26-4.15$ (m, 2H, $\mathrm{CH}_{2}-7$ ), 3.37 (s, 2H, CH-13), 2.56 (br. s, $\left.1 \mathrm{H}, \mathrm{NH}\right), 1.16\left(\mathrm{t}, J=7.1 \mathrm{~Hz}, 3 \mathrm{H}, \mathrm{CH}_{3}-8\right)$; ${ }^{13} \mathrm{C}$ NMR (101 MHz, $\mathrm{CDCl}_{3}$ ): $\delta 172.5$ (C-6), 149.0 (C-4), 147.1 (C-1), 141.0 (C-9), 139.8 (C14), 129.5 (C-3), 128.7 (C-15), 128.6 (C-16), 128.2 (C-11), 128.1 (C-12), 127.7 (C-10), 127.4 (C17), 123.2 (C-2), 72.7 (C-5), 62.2 (C-7), 48.6 (C-13), 14.2 (C-8).

IR (neat film, $\mathrm{cm}^{-1}$ ): 3334, 3062, 2980, 2853, 1728, 1518, 1346, 1220, 1993, 1026, 1015, 853, 733, 699 .

MS $\left(\mathrm{ES}^{+}\right.$) found $\mathrm{m} / \mathrm{z} 391[\mathrm{M}+\mathrm{H}]^{+}$; HRMS $\left(\mathrm{ES}^{+}\right.$) found 391.1652, $\mathrm{C}_{23} \mathrm{H}_{23} \mathrm{~N}_{2} \mathrm{O}_{4}[\mathrm{M}+\mathrm{H}]^{+}$ requires 391.1647 .

Ethyl 2-(benzylamino)-2-(4-nitrophenyl)propanoate 8n

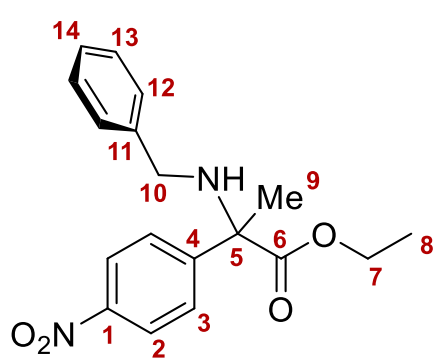

Prepared according to General Procedure E from 4-nitro$\mathrm{N}$-benzylbenzenesulfonamide 60 (0.10 mmol, $29.2 \mathrm{mg})$, $\mathrm{K}_{2} \mathrm{CO}_{3}$ (0.30 mmol, $41.5 \mathrm{mg}$ ), ethyl 2-chloropropionate (o.20 mmol, $25 \mu \mathrm{L})$, DMF (1.0o $\mathrm{mL})$. Purification by column chromatography on silica gel (o-40\% EtOAc in hexanes) afforded $8 \mathbf{n}$ as a light yellow solid $(18.7 \mathrm{mg}$, $0.057 \mathrm{mmol}$, $57 \%)$, m.p. $73^{-} 75^{\circ} \mathrm{C}$.

${ }^{1} \mathrm{H}$ NMR (400 MHz, $\mathrm{CDCl}_{3}$ ): $\delta 8.20$ (app. d, $J=9.0 \mathrm{~Hz}, 2 \mathrm{H}, \mathrm{CH}_{\mathrm{ar}}$-2), 7.73 (app. d, $J=9.0$ $\left.\mathrm{Hz}, 2 \mathrm{H}, \mathrm{CH}_{\mathrm{ar}-3}\right), 7.39-7.32\left(\mathrm{~m}, 4 \mathrm{H}, \mathrm{CH}_{\mathrm{ar}}-12,13\right), 7.30-7.26\left(\mathrm{~m}, 1 \mathrm{H}, \mathrm{CH}_{\mathrm{ar}}-14\right), 4.25(\mathrm{~d}, J=$ $\left.7.2 \mathrm{~Hz}, 2 \mathrm{H}, \mathrm{CH}_{2}-7\right), 3.7 \mathrm{O}(\mathrm{d}, J=12.2 \mathrm{~Hz}, 1 \mathrm{H}, \mathrm{CH}-1 \mathrm{O}), 3.62$ (d, $\left.J=12.2 \mathrm{~Hz}, 1 \mathrm{H}, \mathrm{CH}-1 \mathrm{O}\right), 2.3 \mathrm{O}$ (br. s, $\left.{ }_{1} \mathrm{H}, \mathrm{NH}\right), 1.74\left(\mathrm{~s},{ }_{3} \mathrm{H}, \mathrm{CH}_{3}-9\right), 1.27\left(\mathrm{t}, J=7.2 \mathrm{~Hz},{ }_{3} \mathrm{H}, \mathrm{CH}_{3}-8\right) ;{ }^{13} \mathrm{C}$ NMR (101 MHz, 
$\left.\mathrm{CDCl}_{3}\right): \delta$ 174.2 (C-6), $150.9(\mathrm{C}-4), 147.3$ (C-1), 140.0 (C-11), 128.7 (C-12), $128.3(\mathrm{C}-13), 127.4$ (C-14), 127.2 (C-3), 123.7 (C-2), 65.8 (C-5), 61.8 (C-7), 48.4 (C-10), 25.0 (C-9), 14.3 (C-8).

IR (neat film, $\mathrm{cm}^{-1}$ ): 3106, 1535, 1382, 1361, 1347, 1173, 854, 718, 703, 678.

MS $\left(\mathrm{ES}^{+}\right.$) found $m / z 329[\mathrm{M}+\mathrm{H}]^{+}$; HRMS $\left(\mathrm{ES}^{+}\right.$) found 329.1496, $\mathrm{C}_{18} \mathrm{H}_{21} \mathrm{~N}_{2} \mathrm{O}_{4}[\mathrm{M}+\mathrm{H}]^{+}$ requires 329.1486.

\section{Ethyl 2-(methylamino)-2-(4-nitrophenyl)propanoate 80}

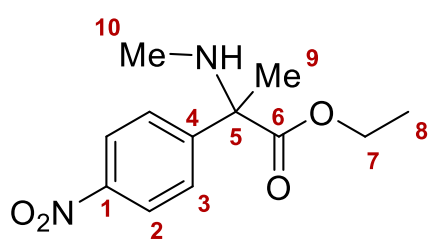

Prepared according to General Procedure E from 4-nitro$N$-methylbenzenesulfonamide 6a (0.10 mmol, $21.6 \mathrm{mg}$ ), $\mathrm{K}_{2} \mathrm{CO}_{3}$ (0.30 mmol, $41.5 \mathrm{mg}$ ), ethyl 2-chloropropionate (o.20 mmol, $25 \mu \mathrm{L})$, DMF (1.0o $\mathrm{mL})$. Purification by column chromatography on silica gel (o-40\% EtOAc in hexanes) afforded 80 as a yellow oil (18.1 mg, 0.0718 mmol, 72\%).

${ }^{1} \mathrm{H}$ NMR (400 MHz, $\mathrm{CDCl}_{3}$ ): $\delta 8.19$ (app. d, $J=9.1 \mathrm{~Hz}, 2 \mathrm{H}, \mathrm{CH}_{\mathrm{ar}}-2$ ), 7.64 (app. d, $J=9.1$ $\left.\mathrm{Hz}, 2 \mathrm{H}, \mathrm{CH}_{\mathrm{ar}}-3\right), 4.22\left(\mathrm{q}, J=7.1 \mathrm{~Hz}, 2 \mathrm{H}, \mathrm{CH}_{2}-7\right), 2.32\left(\mathrm{~s}, 3 \mathrm{H}, \mathrm{CH}_{3}-10\right)$, 2.05 (br. s, $1 \mathrm{H}, \mathrm{NH}$ ), $1.64\left(\mathrm{~s}, 3 \mathrm{H}, \mathrm{CH}_{3}-9\right), 1.24\left(\mathrm{t}, J=7.1 \mathrm{~Hz},{ }_{3} \mathrm{H}, \mathrm{CH}_{3}-8\right) ;{ }^{13} \mathrm{C}$ NMR (101 $\left.\mathrm{MHz}, \mathrm{CDCl}_{3}\right): \delta 174.2$ (C-6), 150.8 (C-4), 147.3 (C-1), 127.2 (C-3), 123.7 (C-2), 66.0 (C-5), 61.8 (C-7), 30.5 (C-10), $23.9(\mathrm{C}-9), 14.3(\mathrm{C}-8)$.

IR (neat film, $\mathrm{cm}^{-1}$ ): 3352, 2982, 2942, 2854, 2803, 1727, 1605, 1520, 1491, 1449, 1347, 1234, 1149, 1109, 1098, 1014, 855, 757, 741, 700.

MS $\left(\mathrm{ES}^{+}\right.$) found $\mathrm{m} / \mathrm{z} 253[\mathrm{M}+\mathrm{H}]^{+}$; HRMS $\left(\mathrm{ES}^{+}\right)$found 253.1176, $\mathrm{C}_{12} \mathrm{H}_{17} \mathrm{~N}_{2} \mathrm{O}_{4}[\mathrm{M}+\mathrm{H}]^{+}$ requires 253.1183 .

\section{Diethyl 2-(methylamino)-2-(4-nitrophenyl)-malonate 8p}<smiles>CCOC(=O)C(NC)(C(=O)OCC)c1ccc([N+](=O)[O-])cc1</smiles>

Diethyl 2-chloromalonate (4.0 eq., $3.0 \mathrm{mmol}, 491 \mu \mathrm{L}$ ) was added to a solution of 4-nitro- $N$ methylbenzenesulfonamide $6 a$ (1.o eq., $0.76 \mathrm{mmol}, 165 \mathrm{mg}$ ), $\mathrm{K}_{2} \mathrm{CO}_{3}$ (3.0 eq., $2.3 \mathrm{mmol}, 318 \mathrm{mg}$ ), DMF (8.0o mL) within a 
metal-capped, oven-dried microwave vial. The reaction was stirred at room temperature for $16 \mathrm{~h}$, after completion EtOAc was added to the mixture and washed with $10 \% \mathrm{LiCl}_{(\mathrm{aq} .)}$. The organic layers were extracted using EtOAc, washed with $\mathrm{H}_{2} \mathrm{O}$ and sat. $\mathrm{NaCl}_{(\text {aq.) }}$, dried using $\mathrm{MgSO}_{4}$, filtered, and concentrated in vacuo to give the crude product. Purification by column chromatography on silica gel (o-40\% EtOAc in hexanes) afforded $\mathbf{8 p}$ as a clear colourless oil (124 $\mathrm{mg}, 0.40 \mathrm{mmol}, 53 \%)$.

${ }^{1} \mathrm{H}$ NMR (400 $\mathrm{MHz}, \mathrm{CDCl}_{3}$ ): $\delta 8.21$ (app. d, $J=9.1 \mathrm{~Hz}, 2 \mathrm{H}, \mathrm{CH}_{\mathrm{ar}}-2$ ), 7.89 (app. d, $J=9.1$ $\mathrm{Hz}, 2 \mathrm{H}, \mathrm{CH}_{\mathrm{ar}}-3$ ), 4.31-4.19 (m, $4 \mathrm{H}, \mathrm{CH}_{2}-7$ ), 2.58 (br. s, $1 \mathrm{H}, \mathrm{NH}$ ), 2.27 (s, ${ }_{3} \mathrm{H}, \mathrm{CH}_{3}-9$ ), 1.26 $\left(\mathrm{t}, J=7.0 \mathrm{~Hz}, 6 \mathrm{H}, \mathrm{CH}_{3}-8\right) ;{ }^{13} \mathrm{C}$ NMR (101 MHz, $\mathrm{CDCl}_{3}$ ): $\delta 168.5$ (C-6), 147.9 (C-4), 142.4 (C-1), 130.2 (C-3), 123.1 (C-2), 73.7 (C-5), 62.5 (C-7), 30.3 (C-9), 14.2 (C-8).

IR (neat film, $\mathrm{cm}^{-1}$ ): 3370, 2983, 2905, 2809, 1732, 1521, 1350, 1298, 1242, 1206, 1152, 1096, 1030, 1016, 856, 737 .

MS $\left(\mathrm{ES}^{+}\right)$found $m / z$ z11 $[\mathrm{M}+\mathrm{H}]^{+}, 333[\mathrm{M}+\mathrm{Na}]^{+}$; HRMS $\left(\mathrm{ES}^{+}\right)$found 311.1230, $\mathrm{C}_{14} \mathrm{H}_{19} \mathrm{~N}_{2} \mathrm{O}_{6}$ $[\mathrm{M}+\mathrm{H}]^{+}$requires 311.1238 .

\section{Ethyl 2-(benzylamino)2-phenyl-2-(pyrimidin-2-yl)acetate 8q}

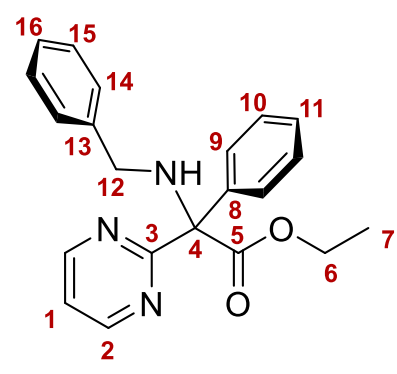

Prepared according to General Procedure E from $N$ benzylpyrimidine-2-sulfonamide (o.10 mmol, $24.9 \mathrm{mg}$ ), $\mathrm{K}_{2} \mathrm{CO}_{3}$ (0.30 mmol, $41.5 \mathrm{mg}$ ), ethyl $\alpha$-chlorophenylacetate 152a (o.20 mmol, $34 \mu \mathrm{L})$, DMF (1.0o $\mathrm{mL})$. Purification by column chromatography on silica gel (o-40\% EtOAc in hexanes) afforded 8q as a light yellow solid (18.9 $\mathrm{mg}$, $0.054 \mathrm{mmol}, 54 \%$ ), $62-64{ }^{\circ} \mathrm{C}$.

${ }^{1} \mathrm{H}$ NMR (500 MHz, $\mathrm{CDCl}_{3}$ ): $\delta 8.72$ (app. d, $J=4.9 \mathrm{~Hz}, 2 \mathrm{H}, \mathrm{CH}_{\mathrm{ar}}-2$ ), 7.79 (app. d, $J=7.4$ $\left.\mathrm{Hz}, 2 \mathrm{H}, \mathrm{CH}_{\mathrm{ar}}-9\right), 7 \cdot 39^{-7.35}\left(\mathrm{~m}, 4 \mathrm{H}, \mathrm{CH}_{\mathrm{ar}}-14,15\right), 7 \cdot 33^{-7.29}\left(\mathrm{~m}, 3 \mathrm{H}, \mathrm{CH}_{\mathrm{ar}}-10,16\right), 7.24$ (app. t, $J=7.3 \mathrm{~Hz}, 1 \mathrm{H}, \mathrm{CH}_{\mathrm{ar}}-11$ ), 7.16 (app. t, $J=4.9 \mathrm{~Hz}, 1 \mathrm{H}, \mathrm{CH}_{\mathrm{ar}-1}$ ), 4.27-4.20 (m, $2 \mathrm{H}$, $\left.\mathrm{CH}_{2}-6\right), 3.71\left(\mathrm{~d}, J=12.9 \mathrm{~Hz},{ }_{1} \mathrm{H}, \mathrm{CH}_{2}-12\right), 3.56$ (d, $\left.J=12.9 \mathrm{~Hz},{ }_{1} \mathrm{H}, \mathrm{CH}_{2}-12\right), 3.31$ (br. s, $1 \mathrm{H}$, $\mathrm{NH}), 1.18\left(\mathrm{t}, J=7.1 \mathrm{~Hz}, 3 \mathrm{H}, \mathrm{CH}_{3}-7\right) ;{ }^{13} \mathrm{C} \mathrm{NMR}\left(126 \mathrm{MHz}, \mathrm{CDCl}_{3}\right): \delta 172.1(\mathrm{C}-5)$, $170.0(\mathrm{C}-3)$, 157.0 (C-2), 140.5 (C-13), 138.5 (C-8), 129.4 (C-9), 128.7 (C-16), 128.5 (C-10), 128.3 (C-15), 127.9 (C-14), 127.0 (C-11), 119.4 (C-1), 75.0 (C-4), 61.6 (C-6), 48.1 (C-12), 14.2 (C-7). 


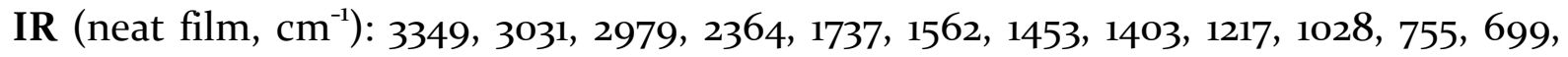
634 .

MS $\left(\mathrm{ES}^{+}\right)$found $m / z 349[\mathrm{M}+\mathrm{H}]^{+} ;$HRMS $\left(\mathrm{APCI}^{+}\right)$found 348.1707, $\mathrm{C}_{21} \mathrm{H}_{22} \mathrm{~N}_{3} \mathrm{O}_{2}[\mathrm{M}+\mathrm{H}]^{+}$ requires 348.1698 .

Ethyl (Z)-2-(4-nitrophenyl)-2-(phenylimino)acetate na<smiles>CCOC(=O)C(=Nc1ccccc1)c1ccc([N+](=O)[O-])cc1</smiles>

Prepared according to General Procedure F from 4nitro- $N$-phenylbenzenesulfonamide $\mathbf{6 g}$ (0.36 mmol, 100 $\mathrm{mg}), \mathrm{K}_{2} \mathrm{CO}_{3}(1.1 \mathrm{mmol}, 152 \mathrm{mg})$, ethyl 2-chloroacetate $\mathbf{7 b}$ (o.72 mmol, $77 \mu \mathrm{L}$ ), DMF (1.0o mL). Purification by column chromatography on silica gel (o-40\% EtOAc in hexanes) afforded 11a as a vibrant yellow solid (121 $\mathrm{mg}$, $0.41 \mathrm{mmol}, 56 \%)$, m.p. $71^{-7} 4^{\circ} \mathrm{C}$. Imine stereochemistry assigned as $\mathrm{Z}$ by comparison with literature data for E-11a. ${ }^{[10]}$

${ }^{1} \mathrm{H}$ NMR (40o MHz, $\mathrm{CDCl}_{3}$ ): $\delta 8.32$ (app. d, $J=8.9 \mathrm{~Hz}, 2 \mathrm{H}, \mathrm{CH}_{\mathrm{ar}}-2$ ), 8.08 (app. d, $J=8.9$ $\mathrm{Hz}, 2 \mathrm{H}, \mathrm{CH}_{\mathrm{ar}}-3$ ), 7.36 (app. dd, $J=8.4,7.5 \mathrm{~Hz}, 2 \mathrm{H}, \mathrm{CH}_{\mathrm{ar}}-11$ ), 7.19 (app. tt, $J=7.5,1.3 \mathrm{~Hz}$, ${ }_{1} \mathrm{H}, \mathrm{CH}_{\mathrm{ar}}-12$ ), 6.97 (app. dd, $\left.J=8.4,1.3 \mathrm{~Hz}, 2 \mathrm{H}, \mathrm{CH}_{\mathrm{ar}}-10\right), 4.16$ (q, $J=7.1 \mathrm{~Hz}, 2 \mathrm{H}, \mathrm{CH}_{2}-7$ ), $1.00\left(\mathrm{t}, J=7.1 \mathrm{~Hz},{ }_{3} \mathrm{H}, \mathrm{CH}_{3}-8\right)$; ${ }^{13} \mathrm{C}$ NMR (101 $\left.\mathrm{MHz} \mathrm{CDCl}_{3}\right): \delta$ 164.2 (C-6), $158.1(\mathrm{C}-5)$, 149.67 (C-4), 149.59 (C-1), 139.4 (C-9), 129.13 (C-3), 129.09 (C-11), 125.8 (C-12), 124.0 (C2), $119.5(\mathrm{C}-10), 62.1(\mathrm{C}-7), 13.8(\mathrm{C}-8)$.

IR (neat film, cm ${ }^{-1}$ ): 3067, 2989, 2922, 2855, 1725, 1617, 1601, 1595, 1579, 1521, 1483, 1464, 1349, 1307, 1301, 1174, 1009, 794, 691.

MS $\left(\mathrm{ES}^{+}\right)$found $m / z 321[\mathrm{M}+\mathrm{Na}]^{+}$; HRMS $\left(\mathrm{ES}^{+}\right)$found 321.0846, $\mathrm{C}_{16} \mathrm{H}_{14} \mathrm{~N}_{2} \mathrm{O}_{4} \mathrm{Na}[\mathrm{M}+\mathrm{Na}]^{+}$ requires 321.0847 . 
Ethyl (Z)-2-(4-nitrophenyl)-2-(p-tolylimino)acetate 11 b

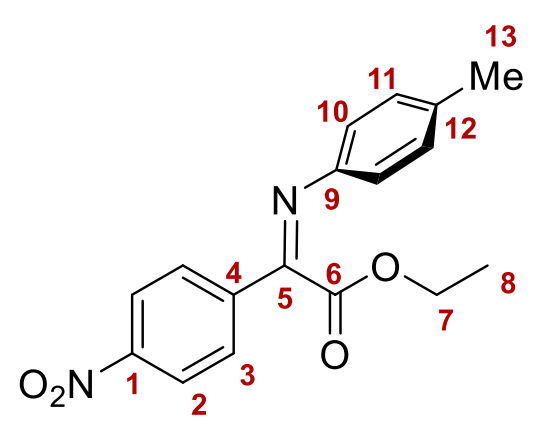

Prepared according to General Procedure F from 4nitro- $N$-(p-tolyl)benzenesulfonamide $6 \mathbf{h}$ (o.10 $\mathrm{mmol}$, $29.2 \mathrm{mg}$ ), $\mathrm{K}_{2} \mathrm{CO}_{3}$ (0.30 mmol, $41.5 \mathrm{mg}$ ), ethyl 2chloroacetate $7 \mathbf{b}(0.20 \mathrm{mmol}, 22 \mu \mathrm{L})$, DMF (1.00 mL). Purification by column chromatography on silica gel (o-40\% EtOAc in hexanes) afforded $\mathbf{1 1 b}$ as a clear brown oil (21.6 mg, o.069 mmol, 69\%).

${ }^{1} \mathrm{H}$ NMR (400 MHz, $\mathrm{CDCl}_{3}$ ): $\delta 8.31$ (app. d, $J=9.0 \mathrm{~Hz}, 2 \mathrm{H}, \mathrm{CH}_{\mathrm{ar}}-2$ ), 8.06 (app. d, $J=9.0$ $\mathrm{Hz}, 2 \mathrm{H}, \mathrm{CH}_{\mathrm{ar}}-3$ ), 7.16 (app. d, $J=8.1 \mathrm{~Hz}, 2 \mathrm{H}, \mathrm{CH}_{\mathrm{ar}}-11$ ), 6.89 (app. d, $J=8.1 \mathrm{~Hz}, 2 \mathrm{H}, \mathrm{CH}_{\mathrm{ar}^{-}}$ 10), 4.19 (q, $\left.J=7.1 \mathrm{~Hz}, 2 \mathrm{H}, \mathrm{CH}_{2}-7\right), 2.35$ (s, $\left.3 \mathrm{H}, \mathrm{CH}-13\right), 1.06\left(\mathrm{t}, J=7.1 \mathrm{~Hz}, 3 \mathrm{H}, \mathrm{CH}_{3}-8\right) ;{ }^{13} \mathrm{C}$ NMR (101 MHz, CDCl $): \delta 164.6$ (C-6), 157.6 (C-5), 149.6 (C-4), 147.0 (C-12), 139.7 (C-1), 135.8 (C-9), 129.7 (C-11), 129.0 (C-3), 124.0 (C-2), 119.7 (C-10), 62.1 (C-7), 21.1 (C-13), 13.9 (C-8).

IR (neat film, $\mathrm{cm}^{-1}$ ): 3082, 3024, 2982, 2923, 2860, 1728, 1521, 1502, 1343, 1320, 1303, 1224, 1188, 1170, 1022, 1012, 863, 850, 818, 698.

MS $\left(\mathrm{ES}^{+}\right.$) found $m / z 335[\mathrm{M}+\mathrm{Na}]^{+}$; HRMS $\left(\mathrm{ES}^{+}\right)$found 335.0998, $\mathrm{C}_{17} \mathrm{H}_{16} \mathrm{~N}_{2} \mathrm{O}_{4} \mathrm{Na}[\mathrm{M}+\mathrm{Na}]^{+}$ requires 335.1002 .

\section{Ethyl (Z)-2-(mesitylimino)-2-(4-nitrophenyl)acetate $11 \mathrm{C}$}

Prepared according to General Procedure F from 4-nitro- $N$ -

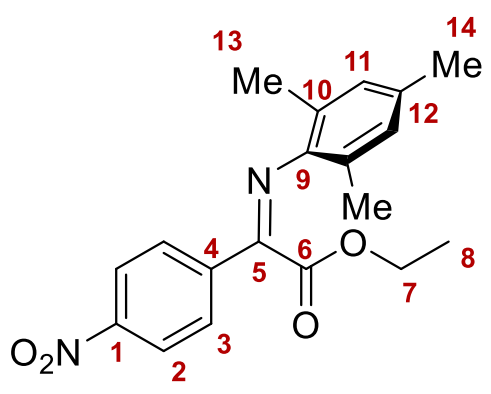
mesitylbenzenesulfonamide $6 \mathbf{i}$ ( $0.10 \mathrm{mmol}, 32.0 \mathrm{mg}$ ), $\mathrm{K}_{2} \mathrm{CO}_{3}(0.30 \mathrm{mmol}, 41.5 \mathrm{mg}$ ), ethyl 2-chloroacetate $\mathbf{7 b}$ (o.20 mmol, $22 \mu \mathrm{L}$ ), DMF (1.0o mL). Purification by column chromatography on silica gel (o-40\% EtOAc in hexanes) afforded 11c as a red-orange solid (25.4 $\mathrm{mg}, 0.075$ mmol, $75 \%)$, m.p. $68-69{ }^{\circ} \mathrm{C}$.

H NMR (40o $\mathrm{MHz}, \mathrm{CDCl}_{3}$ ): $\delta 8.33$ (app. d, $J=9.0 \mathrm{~Hz}, 2 \mathrm{H}, \mathrm{CH}_{\mathrm{ar}}-2$ ), 8.11 (app. d, $J=9.0$ $\left.\mathrm{Hz}, 2 \mathrm{H}, \mathrm{CH}_{\mathrm{ar}}-3\right), 6.82\left(\mathrm{~s}, 2 \mathrm{H}, \mathrm{CH}_{\mathrm{ar}}-11\right), 4.10\left(\mathrm{q}, J=7.1 \mathrm{~Hz}, 2 \mathrm{H}, \mathrm{CH}_{2}-7\right), 2.26\left(\mathrm{~s}, 3 \mathrm{H}, \mathrm{CH}_{3^{-}}\right.$ 14), $2.04\left(\mathrm{~s}, 6 \mathrm{H}, \mathrm{CH}_{3}-13\right), 0.95\left(\mathrm{t}, J=7.1 \mathrm{~Hz}, 3 \mathrm{H}, \mathrm{CH}_{3}-8\right) ;{ }^{13} \mathrm{C}$ NMR (101 $\left.\mathrm{MHz}, \mathrm{CDCl}_{3}\right): \delta$ 
164.1 (C-6), 159.5 (C-5), 149.8 (C-4), 145.3 (C-9), 139.0 (C-1), 133.7 (C-12), 128.9 (C-3), 128.4 (C-11), 125.7 (C-10), 124.0 (C-2), 61.9 (C-7), 20.9 (C-14), 18.0 (C-13), 13.7(C-8).

IR (neat film, $\mathrm{cm}^{-1}$ ): 2980, 2917, 2857, 1732, 1601, 1523, 1341, 1304, 1216, 1193, 1143, 1021, $1012,853,701$.

MS $\left(\mathrm{ES}^{+}\right)$found $m / z 363[\mathrm{M}+\mathrm{Na}]^{+}$; HRMS $\left(\mathrm{ES}^{+}\right.$) found 363.1312, $\mathrm{C}_{19} \mathrm{H}_{20} \mathrm{~N}_{2} \mathrm{O}_{4} \mathrm{Na}[\mathrm{M}+\mathrm{Na}]^{+}$ requires 363.1315 .

Ethyl (Z)-2-((4-methoxyphenyl)imino)-2-(4-nitrophenyl)acetate 11d

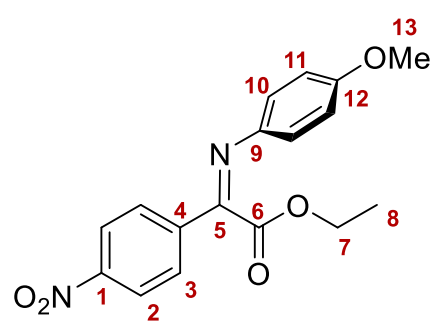

Prepared according to General Procedure F from 4-nitro- $N$ (4-methoxyphenyl)benzenesulfonamide 6j (o.10 mmol, 30.8 $\mathrm{mg}$ ), $\mathrm{K}_{2} \mathrm{CO}_{3}$ (0.30 mmol, $41.5 \mathrm{mg}$ ), ethyl 2-chloroacetate $\mathbf{7 b}$ (0.20 mmol, $22 \mu \mathrm{L}$ ), DMF (1.00 mL). Purification by column chromatography on silica gel (o-40\% EtOAc in hexanes) afforded 11 as a vibrant orange oil (16.8 mg, $0.051 \mathrm{mmol}, 51 \%)$.

${ }^{1}$ H NMR (40o MHz, $\mathrm{CDCl}_{3}$ ): $\delta 8.30$ (app. d, $J=9.0 \mathrm{~Hz}, 2 \mathrm{H}, \mathrm{CH}_{\mathrm{ar}^{-2}}$ ), 8.05 (app. d, $J=9.0$ $\mathrm{Hz}, 2 \mathrm{H}, \mathrm{CH}_{\mathrm{ar}-3}$ ), 7.00 (app. d, $J=8.9 \mathrm{~Hz}, 2 \mathrm{H}, \mathrm{CH}_{\mathrm{ar}}-11$ ), 6.90 (app. d, $J=8.9 \mathrm{~Hz}, 2 \mathrm{H}$, $\left.\mathrm{CH}_{\mathrm{ar}-10}\right), 4.23$ (q, J = 7.2 Hz, $\left.2 \mathrm{H}, \mathrm{CH}_{2}-7\right), 3.82\left(\mathrm{~s}, 3 \mathrm{H}, \mathrm{CH}_{3}-13\right), 1.11\left(\mathrm{t}, J=7.2 \mathrm{~Hz}, 3 \mathrm{H}, \mathrm{CH}_{3^{-}}\right.$ 8); ${ }^{13} \mathrm{C}$ NMR (101 MHz, CDCl 3 ): $\delta 165.0$ (C-6), 158.2 (C-12), 156.8 (C-5), 149.5 (C-4), 142.5 (C-9), 139.8 (C-1), 128.9 (C-3), 124.0 (C-2), 121.6 (C-11), 114.4 (C-10), 62.1 (C-7), 55.6 (C-13), $14.0(\mathrm{C}-8)$.

IR (neat film, $\mathrm{cm}^{-1}$ ): 3108, 3077, 2981, 2937, 2838, 1728, 1600, 1521, 1503, 1345, 1295, 1246, $1226,1189,1163,1022,1012,851,830,700$.

MS $\left(\mathrm{ES}^{+}\right)$found $m / z 351[\mathrm{M}+\mathrm{Na}]^{+} ; \mathrm{HRMS}\left(\mathrm{ES}^{+}\right)$found 351.0944, $\mathrm{C}_{17} \mathrm{H}_{16} \mathrm{~N}_{2} \mathrm{O}_{5} \mathrm{Na}[\mathrm{M}+\mathrm{Na}]^{+}$ requires 351.0951. 
Ethyl (Z)-2-((4-chlorophenyl)imino)-2-(4-nitrophenyl)acetate ne

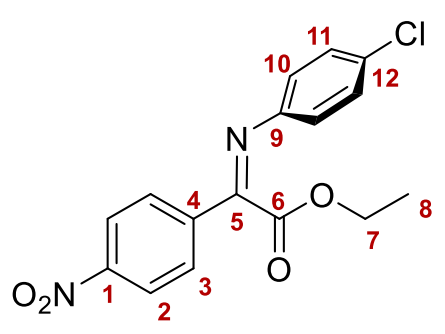

Prepared according to General Procedure F from 4-nitro$\mathrm{N}$-(4-chlorophenyl)benzenesulfonamide 6k (o.10 mmol, 31.3 $\mathrm{mg}), \mathrm{K}_{2} \mathrm{CO}_{3}(0.30 \mathrm{mmol}, 41.5 \mathrm{mg})$, ethyl 2-chloroacetate $\mathbf{7 b}$ (o.20 mmol, $22 \mu \mathrm{L}$ ), DMF (1.0o mL). Purification by column chromatography on silica gel (o-40\% EtOAc in hexanes) afforded 11e as a yellow oil (16.7 mg, 0.050 mmol, 50\%).

${ }^{1} \mathrm{H}$ NMR (400 MHz, $\mathrm{CDCl}_{3}$ ): $\delta 8.25$ (app. d, $J=8.8 \mathrm{~Hz}, 2 \mathrm{H}, \mathrm{CH}_{\mathrm{ar}}-2$ ), 7.99 (app. d, $J=8.8$ $\mathrm{Hz}, 2 \mathrm{H}, \mathrm{CH}_{\mathrm{ar}}-3$ ), 7.25 (app. d, $J=8.4 \mathrm{~Hz}, 2 \mathrm{H}, \mathrm{CH}_{\mathrm{ar}}-11$ ), 6.85 (app. d, $J=8.4 \mathrm{~Hz}, 2 \mathrm{H}, \mathrm{CH}_{\mathrm{ar}^{-}}$ 10), 4.13 (q, $\left.J=7.1 \mathrm{~Hz}, 2 \mathrm{H}, \mathrm{CH}_{2}-7\right)$, $1.00\left(\mathrm{t}, J=7.1 \mathrm{~Hz}, 3 \mathrm{H}, \mathrm{CH}_{3}-8\right) ;{ }^{13} \mathrm{C}$ NMR (101 MHz, $\mathrm{CDCl}_{3}$ ): $\delta$ 164.0 (C-6), 158.6 (C-5), 149.8 (C-4), 148.0 (C-9), 139.1 (C-1), 131.4 (C-12), 129.22 (C-3), 129.18 (C-10), 124.0 (C-2), 121.0 (C-11), 62.4 (C-7), 13.9 (C-8).

IR (neat film, $\mathrm{cm}^{-1}$ ): 3320, 3112, 3087, 2987, 2962, 2940, 2857, 1714, 1522, 1479, 1347, 1309, 1229, 1186, 1168, 1090, 1021, 1012, 851, 836, 752, 704, 696, 664.

MS $\left(\mathrm{ES}^{+}\right.$) found $\mathrm{m} / \mathrm{z} 333[\mathrm{M}+\mathrm{H}]^{+}$; HRMS $\left(\mathrm{ES}^{+}\right.$) found 333.0637, $\mathrm{C}_{16} \mathrm{H}_{14} \mathrm{ClN}_{2} \mathrm{O}_{4}[\mathrm{M}+\mathrm{H}]^{+}$ requires 333.0637 .

Ethyl (Z)-4-((2-ethoxy-1-(4-nitrophenyl)-2-oxoethylidene)imino)benzoate 11 f

Prepared according to General Procedure F from ethyl 4-((4-

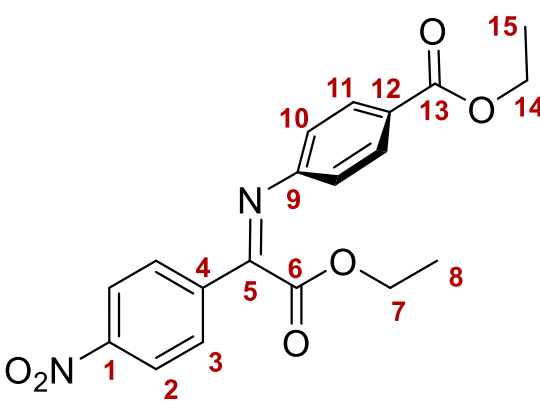
nitrophenyl)sulfonamido)benzoate 61 (o.10 mmol, $35.0 \mathrm{mg}), \mathrm{K}_{2} \mathrm{CO}_{3}$ (0.30 mmol, $41.5 \mathrm{mg}$ ), ethyl 2chloroacetate $\mathbf{7 b}$ (0.20 mmol, $22 \mu \mathrm{L})$, DMF (1.00 mL). Purification by column chromatography on silica gel (o-40\% EtOAc in hexanes) afforded nf as a yellow solid (13.5 mg, $0.037 \mathrm{mmol}, 37 \%)$, m.p. $76-79{ }^{\circ} \mathrm{C}$.

${ }^{1} \mathrm{H}$ NMR (40o MHz, $\mathrm{CDCl}_{3}$ ): $\delta 8.33$ (app. d, $J=9.0 \mathrm{~Hz}, 2 \mathrm{H}, \mathrm{CH}_{\mathrm{ar}}-2$ ), 8.09 (app. d, $J=9.0$ $\mathrm{Hz}, 2 \mathrm{H}, \mathrm{CH}_{\mathrm{ar}-3}$ ), 8.06 (app. d, $J=8.6 \mathrm{~Hz}, 2 \mathrm{H}, \mathrm{CH}_{\mathrm{ar}-10}$ ), 6.99 (app. d, $J=8.6 \mathrm{~Hz}, 2 \mathrm{H}$, $\left.\mathrm{CH}_{\mathrm{ar}}-11\right), 4.38$ (q, $\left.J=7.2 \mathrm{~Hz}, 2 \mathrm{H}, \mathrm{CH}_{2}-14\right), 4.15$ (q, $\left.J=7.1 \mathrm{~Hz}, 2 \mathrm{H}, \mathrm{CH}_{2}-7\right), 1.40(\mathrm{t}, J=7.2$ $\left.\mathrm{Hz},{ }_{3} \mathrm{H}, \mathrm{CH}_{3}-15\right)$, $1.01\left(\mathrm{t}, J=7.1 \mathrm{~Hz},{ }_{3} \mathrm{H}, \mathrm{CH}_{3}-8\right) ;{ }^{13} \mathrm{C} \mathrm{NMR}\left(101 \mathrm{MHz}, \mathrm{CDCl}_{3}\right): \delta 166.3(\mathrm{C}-$ 
13), 163.5 (C-6), 158.7 (C-5), 153.5 (C-9), 149.9 (C-4), 138.9 (C-1), 130.8 (C-10), 129.3 (C-3), 127.7 (C-12), 124.1 (C-2), 119.2 (C-11), 62.4 (C-7), 61.2 (C-14), 14.5 (C-15), 13.9 (C-8).

IR (neat film, $\mathrm{cm}^{-1}$ ): 2981, 2936, 2355, 1713, 1601, 1524, 1347, 1272, 1226, 1190, 1166, 1101, $1021,1013,858,697$.

MS $\left(\mathrm{ES}^{+}\right)$found $\mathrm{m} / \mathrm{z} 393[\mathrm{M}+\mathrm{Na}]^{+}$; HRMS $\left(\mathrm{ES}^{+}\right.$) found 393.1056, $\mathrm{C}_{19} \mathrm{H}_{18} \mathrm{~N}_{2} \mathrm{O}_{6} \mathrm{Na}[\mathrm{M}+\mathrm{Na}]^{+}$ requires 393.1057.

Ethyl (E)-2-amino-4-(4-nitrophenyl)-2-phenylbut-3-enoate $14 a$

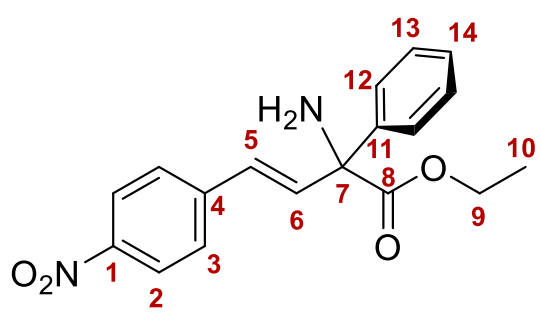

Prepared following General Procedure E using 2-(4nitrophenyl)ethylene-1-sulfonamide $13 a$ (0.20 mmol, $45.6 \mathrm{mg}$ ), $\mathrm{K}_{2} \mathrm{CO}_{3}$ (o.6o mmol, 83.0 mg), ethyl $\alpha-$ chlorophenylacetate (0.40 mmol, $69 \mu \mathrm{L})$, DMF (2.0 $\mathrm{mL})$, after purification by column chromatography on silica gel (10-80\% EtOAc in hexane) to give 14a as a yellow oil (23.0 mg, 0.070 mmol, $35 \%$ yield).

${ }^{1} \mathrm{H}$ NMR (400 MHz, $\left.\left(\mathrm{CD}_{3}\right)_{2} \mathrm{CO}\right): \delta 8.22\left(\mathrm{~d}, J=8.8 \mathrm{~Hz}, 2 \mathrm{H}, \mathrm{CH}_{\mathrm{ar}}-2\right), 7.77(\mathrm{~d}, J=8.8 \mathrm{~Hz}$, $\left.2 \mathrm{H}, \mathrm{CH}_{\mathrm{ar}}-3\right), 7.53\left(\mathrm{~d}, 2 \mathrm{H}, J=7.5 \mathrm{~Hz}, \mathrm{CH}_{\mathrm{ar}}-12\right), 7.37\left(\mathrm{t}, J=7.5 \mathrm{~Hz}, 2 \mathrm{H}, \mathrm{CH}_{\mathrm{ar}^{-13}}\right), 7.29(\mathrm{~m}$, $\left.{ }_{1} \mathrm{H}, \mathrm{CH}_{\mathrm{ar}}-14\right), 7.15(\mathrm{~d}, J=15.5 \mathrm{~Hz}, 1 \mathrm{H}, \mathrm{CH}-5), 7.03\left(\mathrm{~d}, J=15.5 \mathrm{~Hz},{ }_{1} \mathrm{H}, \mathrm{CH}-6\right), 4.23$ (qd, $J=$ 7.2 Hz, 2.2 Hz, 2H, $\mathrm{CH}_{2}-9$ ), 2.49 (br. s, $\left.1 \mathrm{H}, \mathrm{NH}\right), 1.23$ (t, J = 7.2 Hz, $\left.3 \mathrm{H}, \mathrm{CH}_{3}-10\right) .{ }^{13} \mathrm{C}$ NMR (101 MHz, $\left.\left(\mathrm{CD}_{3}\right)_{2} \mathrm{CO}\right): \delta 174.8$ (C-8), 147.9 (C-1), 144.7 (C-4), 144.4 (C-11), 138.5 (C-6), 129.3 (C-13), 128.4 (C-5), 128.4 (C-14), 128.2 (C-2), 127.0 (C-12), 124.7 (C-3), $66.4(\mathrm{C}-7)$, $62.28(\mathrm{C}-9), 14.4(\mathrm{C}-10)$. HRMS $\left(\mathrm{APCI}^{+}\right)$found 327.1323, $\mathrm{C}_{18} \mathrm{H}_{19} \mathrm{~N}_{2} \mathrm{O}_{4}[\mathrm{M}+\mathrm{H}]^{+}$requires 327.1339 .

Ethyl (E)-2-methlyamino-4-(4-nitrophenyl)-2-phenylbut-3-enoate 14b

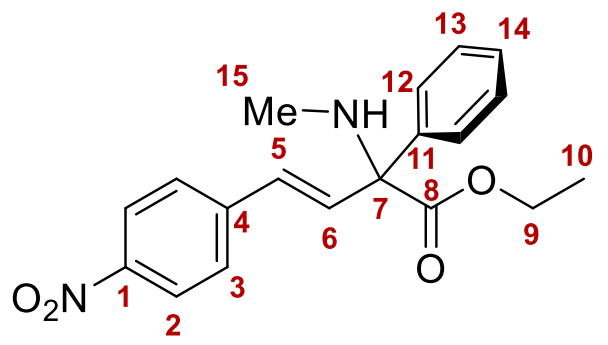

Prepared following General Procedure E using Nmethyl-2-(4-nitrophenyl)ethylene-1-sulfonamide 13 b (o.20 mmol, $48.4 \mathrm{mg}$ ), $\mathrm{K}_{2} \mathrm{CO}_{3}$ (o.6o mmol, 83.0 $\mathrm{mg}$ ), ethyl $\alpha$-chlorophenylacetate (o.40 mmol, 69 
$\mu \mathrm{L})$, DMF (2.0o $\mathrm{mL}$ ), after purification by column chromatography on silica gel (10$80 \%$ EtOAc in hexane) to give $\mathbf{1 4} \mathbf{b}$ as a yellow oil (40.1 $\mathrm{mg}$, $0.12 \mathrm{mmol}$, $59 \%$ yield).

${ }^{1} \mathrm{H}$ NMR (400 MHz, $\left.\left(\mathrm{CD}_{3}\right)_{2} \mathrm{CO}\right): \delta 8.21\left(\mathrm{~d}, J=8.8 \mathrm{~Hz}, 2 \mathrm{H}, \mathrm{CH}_{\mathrm{ar}}-2\right), 7.77(\mathrm{~d}, J=8.8 \mathrm{~Hz}$, $\left.2 \mathrm{H}, \mathrm{CH}_{\mathrm{ar}}-3\right), 7.48\left(\mathrm{~m}, 2 \mathrm{H}, \mathrm{CH}_{\mathrm{ar}}-12\right), 7.37\left(\mathrm{t}, J=7.5 \mathrm{~Hz}, 2 \mathrm{H}, \mathrm{CH}_{\mathrm{ar}}-13\right), 7.29\left(\mathrm{~m}, 1 \mathrm{H}, \mathrm{CH}_{\mathrm{ar}}{ }^{-}\right.$ 14), $7.06(\mathrm{~d}, J=15.5 \mathrm{~Hz}, 1 \mathrm{H}, \mathrm{CH}-5), 6.95(\mathrm{~d}, J=15.5 \mathrm{~Hz}, 1 \mathrm{H}, \mathrm{CH}-6), 4.25(\mathrm{qd}, J=7.2 \mathrm{~Hz}$, $\left.2.2 \mathrm{~Hz}, 2 \mathrm{H}, \mathrm{CH}_{2}-9\right), 2.66$ (bs, $\left.1 \mathrm{H}, \mathrm{NH}\right), 2.27\left(\mathrm{~s}, 3 \mathrm{H}, \mathrm{CH}_{3}-15\right), 1.22\left(\mathrm{t}, J=7.2 \mathrm{~Hz}, 3 \mathrm{H}, \mathrm{CH}_{3}-\right.$ 10). ${ }^{13} \mathrm{C}$ NMR (101 MHz, $\left.\left(\mathrm{CD}_{3}\right)_{2} \mathrm{CO}\right): \delta 172.9$ (C-8), $147.0(\mathrm{C}-1), 143.8(\mathrm{C}-4), 141.6(\mathrm{C}-11)$, 135.1 (C-6), 128.5 (C-5), 128.4 (C-13), 127.5 (C-14), 127.4 (C-2), 126.7 (C-12), 123.8 (C-3), 70.7 (C-7), 61.1 (C-9), 42.3 (C-15), $13.6(\mathrm{C}-10)$. HRMS ( $\left.\mathrm{APCI}^{+}\right)$found 341.1488, $\mathrm{C}_{19} \mathrm{H}_{21} \mathrm{~N}_{2} \mathrm{O}_{4}$ $[\mathrm{M}+\mathrm{H}]^{+}$requires 341.1496.

\section{Reduction of Imine to Arylglycine Derivative}

\section{Ethyl 2-(4-aminophenyl)-2-(phenylamino)acetate 12a}

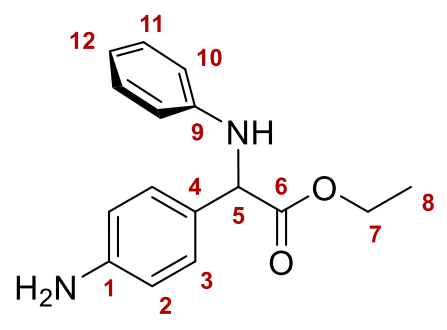

Method adapted from Mangas-Sánchez et al. ${ }^{[1]} \mathrm{NiCl}_{2} \cdot 6 \mathrm{H}_{2} \mathrm{O}$ (1.5 eq., $0.17 \mathrm{mmol}, 40.4 \mathrm{mg}$ ) was added to a solution of ethyl 2-(4-nitrophenyl)-2-(phenylimino)acetate 11 (1.0 eq., 0.11 mmol, $33.8 \mathrm{mg})$ in $\mathrm{EtOH}(1.00 \mathrm{~mL})$. The solution was cooled to ${ }^{\circ}{ }^{\circ} \mathrm{C}$ and $\mathrm{NaBH}_{4}$ (4.0 eq., $0.44 \mathrm{mmol}, 16.6 \mathrm{mg}$ ) added. After 15 mins the reaction was allowed to warm to room temperature and stirred for $16 \mathrm{~h}$. The mixture was filtered over Celite ${ }^{\circledR}$, the solvents removed under reduced pressure. Purification by column chromatography on silica gel (o-40\% EtOAc in hexanes) afforded 12a as a light yellow oil (21.4 mg, 0.079 mmol, 72\%).

${ }^{1} \mathrm{H}$ NMR (50o MHz, $\mathrm{CDCl}_{3}$ ): $\delta 7.26$ (app. d, $J=8.4 \mathrm{~Hz}, 2 \mathrm{H}, \mathrm{CH}_{\mathrm{ar}}-2$ ), 7.12 (app. dd, $J=8.5,7.5 \mathrm{~Hz}$, 2H, $\mathrm{CH}_{\mathrm{ar}}-11$ ), 6.69 (app. tt, $J=7.5,1.2 \mathrm{~Hz}, 1 \mathrm{H}, \mathrm{CH}_{\mathrm{ar}}-12$ ), 6.65 (app. d, $J=8.4 \mathrm{~Hz}, 2 \mathrm{H}, \mathrm{CH}_{\mathrm{ar}}-3$ ), 6.57 (app. dd, $J=8.5,1.2 \mathrm{~Hz}, 2 \mathrm{H}, \mathrm{CH}_{\mathrm{ar}}-10$ ), 4.95 (s, $1 \mathrm{H}, \mathrm{CH}-5$ ), 4.83 (br. s, $1 \mathrm{H}, \mathrm{NH}$ ), 4.26-4.19 (m, $1 \mathrm{H}$, $\mathrm{CH}_{2}-7$ ), 4.16-4.10 (m, $\left.1 \mathrm{H}, \mathrm{CH}_{2}-7\right), 3.68$ (br. s, $\left.2 \mathrm{H}, \mathrm{NH}_{2}\right), 1.22\left(\mathrm{t}, J=7.1 \mathrm{~Hz},{ }_{3} \mathrm{H}, \mathrm{CH}_{3}-8\right) ;{ }^{13} \mathrm{C} \mathrm{NMR}$ $\left(126 \mathrm{MHz}, \mathrm{CDCl}_{3}\right.$ ): $\delta 172.5$ (C-6), 146.5 (C-1), 146.3 (C-9), 129.3 (C-10), 128.4 (C-2), 127.5 (C-4), 118.0 (C-12), 115.4 (C-3), 113.5 (C-11), 61.7 (C-7), 60.4 (C-5), 14.2 (C-8).

Data is in accordance with previous literature reports. ${ }^{[12]}$ 
5. NMR Spectra

$6 c$

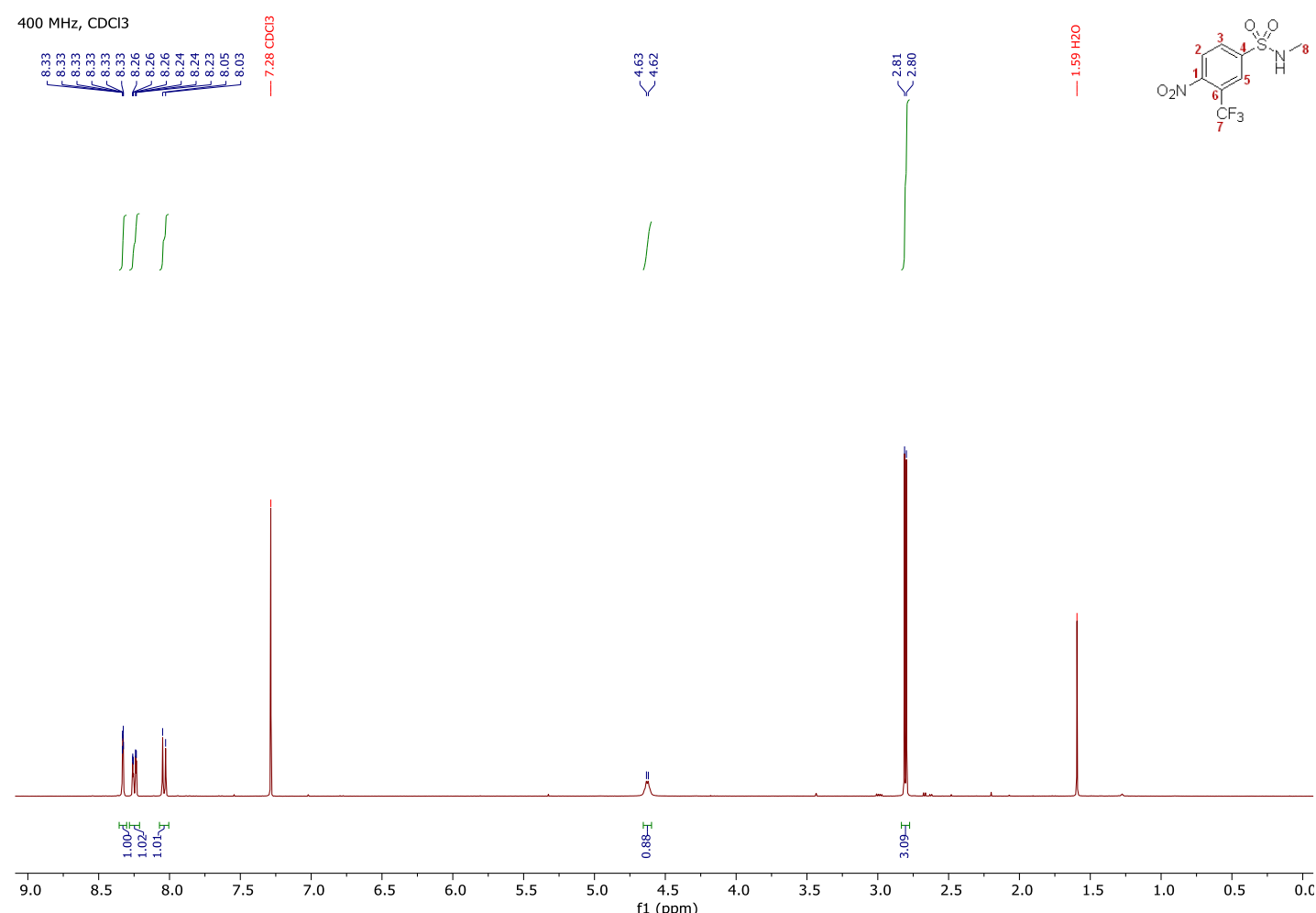

$101 \mathrm{MHz}, \mathrm{CDCl} 3$

| |

$\stackrel{\substack{n \\ \text { I }}}{\text { I }}$

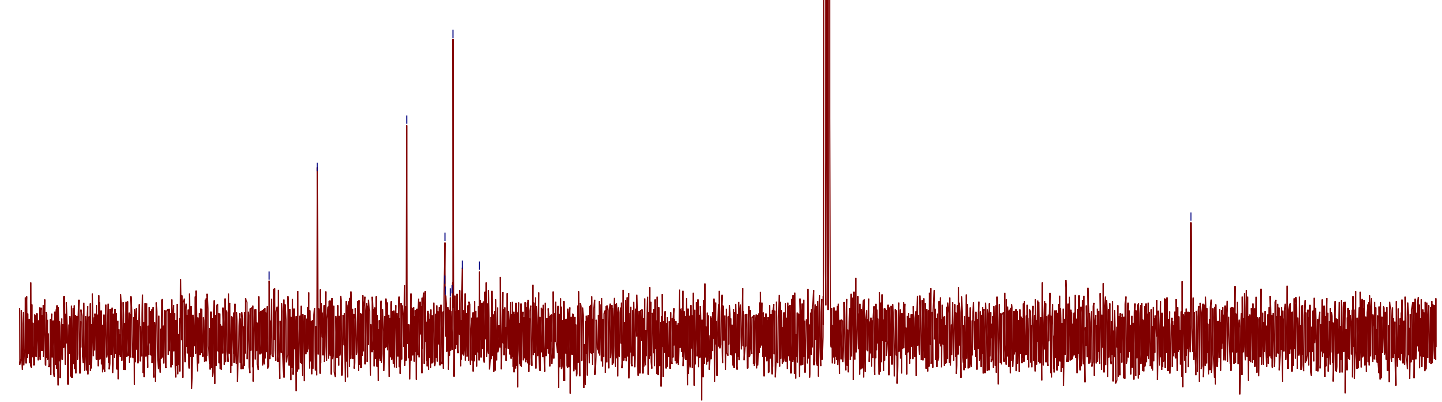

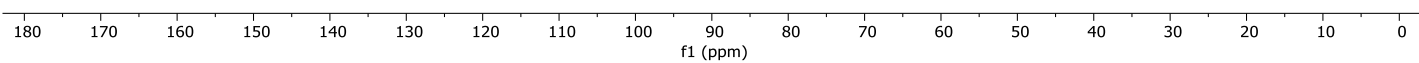


$376 \mathrm{MHz}, \mathrm{CDCl} 3$

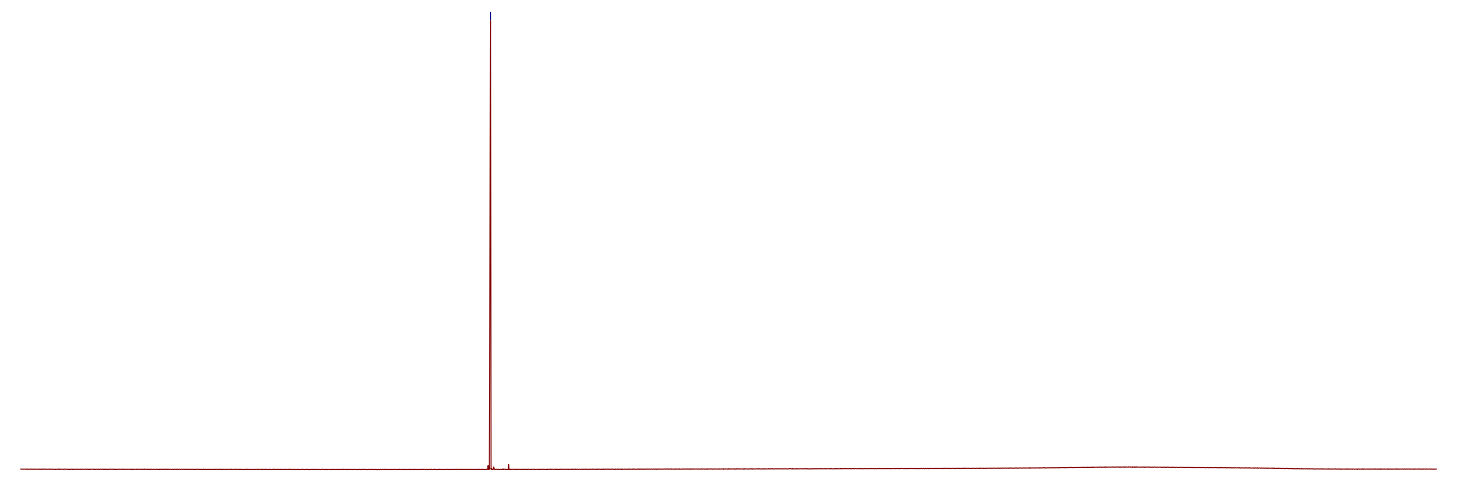

\begin{tabular}{rllllllllllllllllllllllll}
\hline 10 & 0 & -10 & -20 & -30 & -40 & -50 & -60 & -70 & -80 & -90 & -100 & -110 & -120 & -130 & -140 & -150 & -160 & -170 & -180 & -190 & -200 & -210
\end{tabular} 
$6 d$
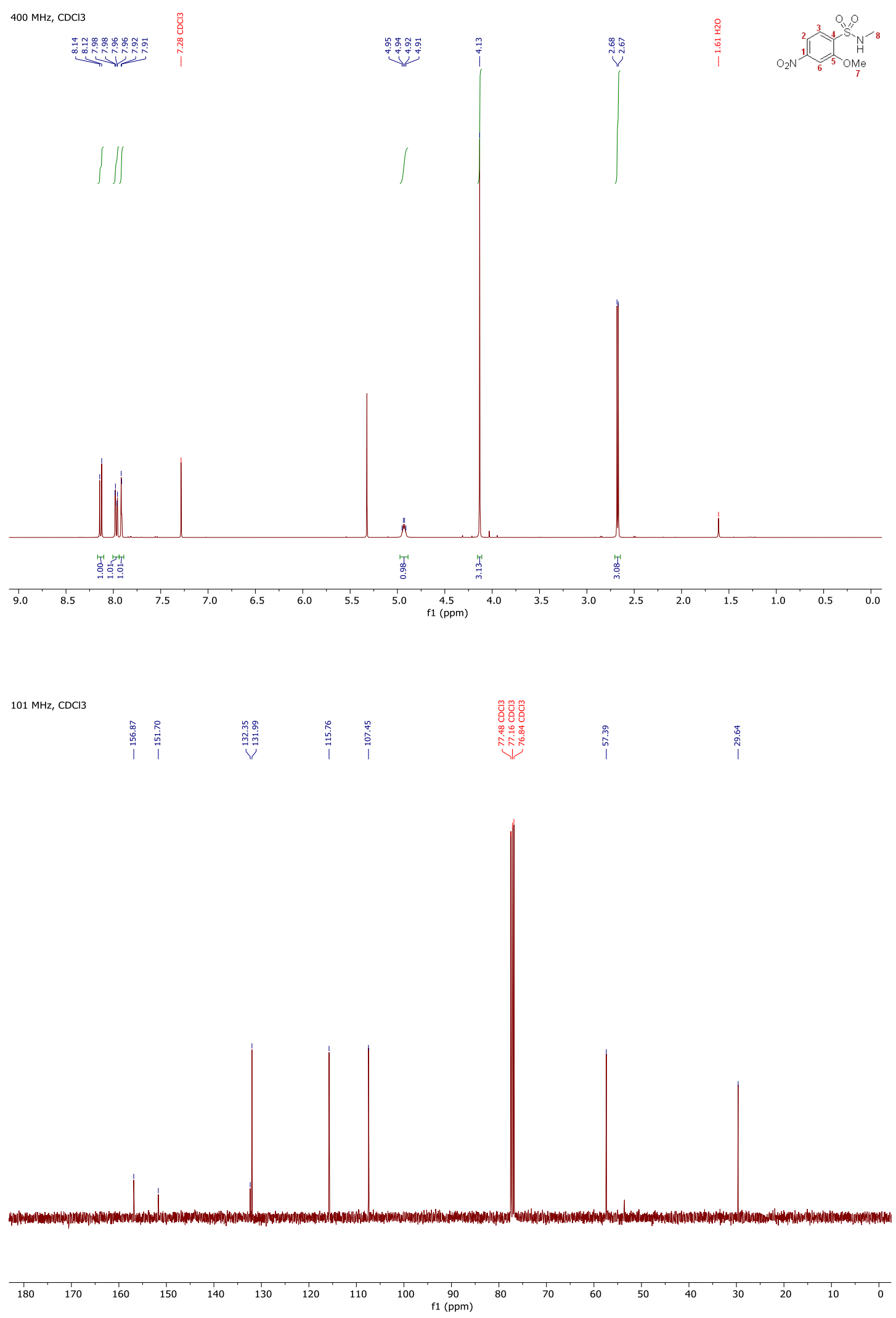

S33 
$6 i$

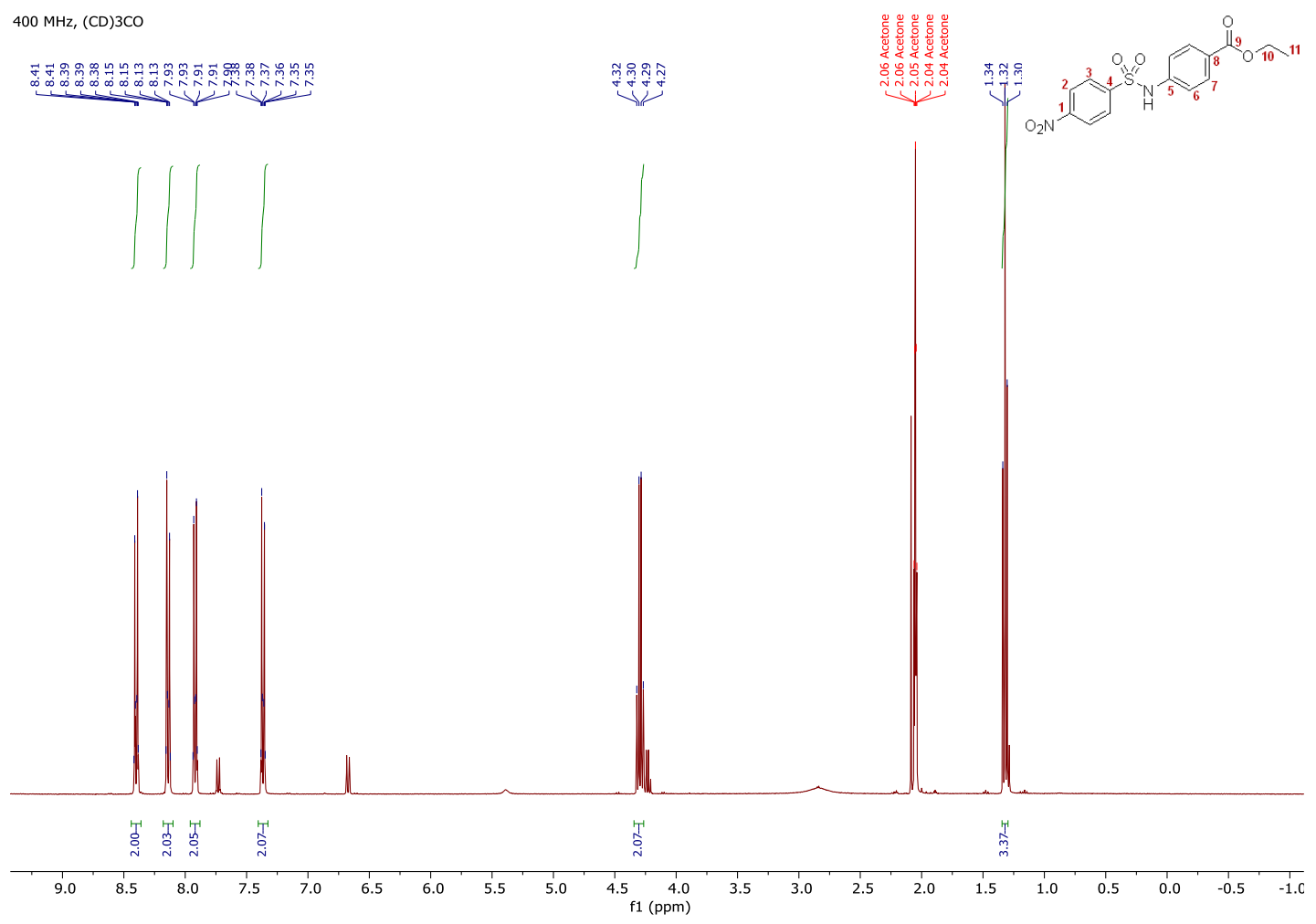

$101 \mathrm{MHz}$, (CD)3CO

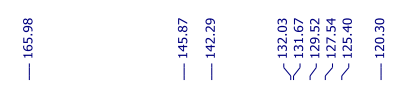
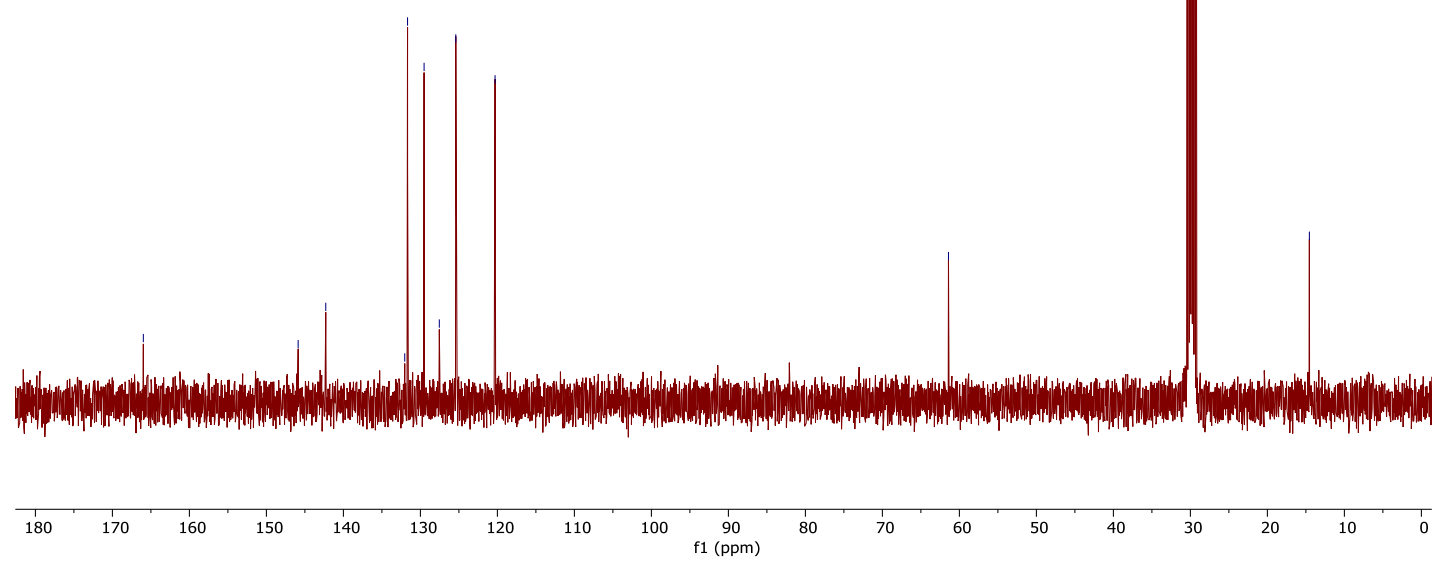

S34 


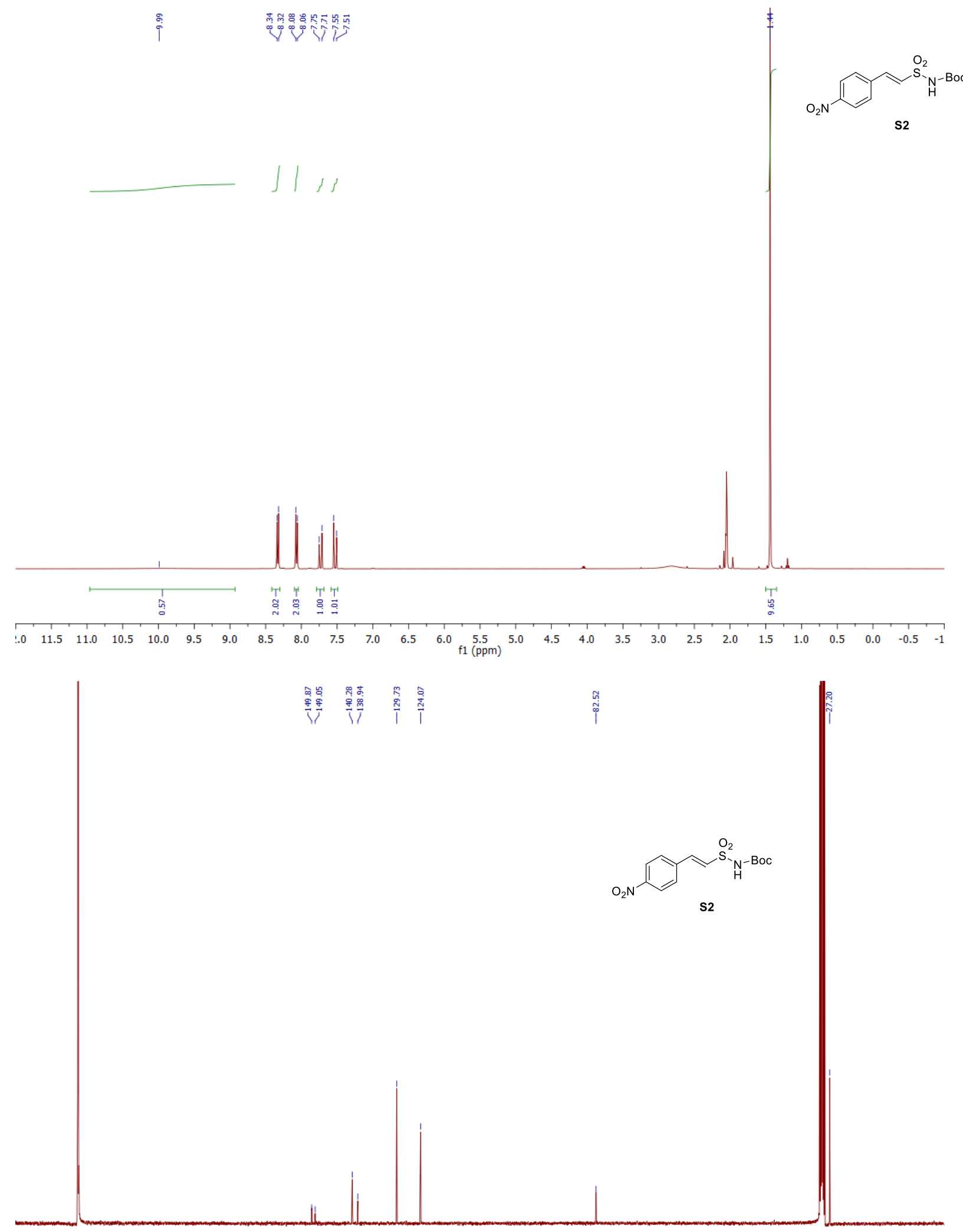

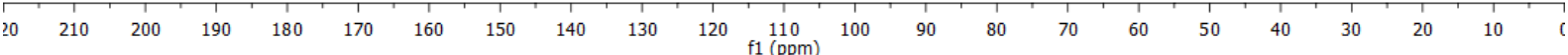




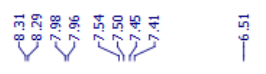

|l f
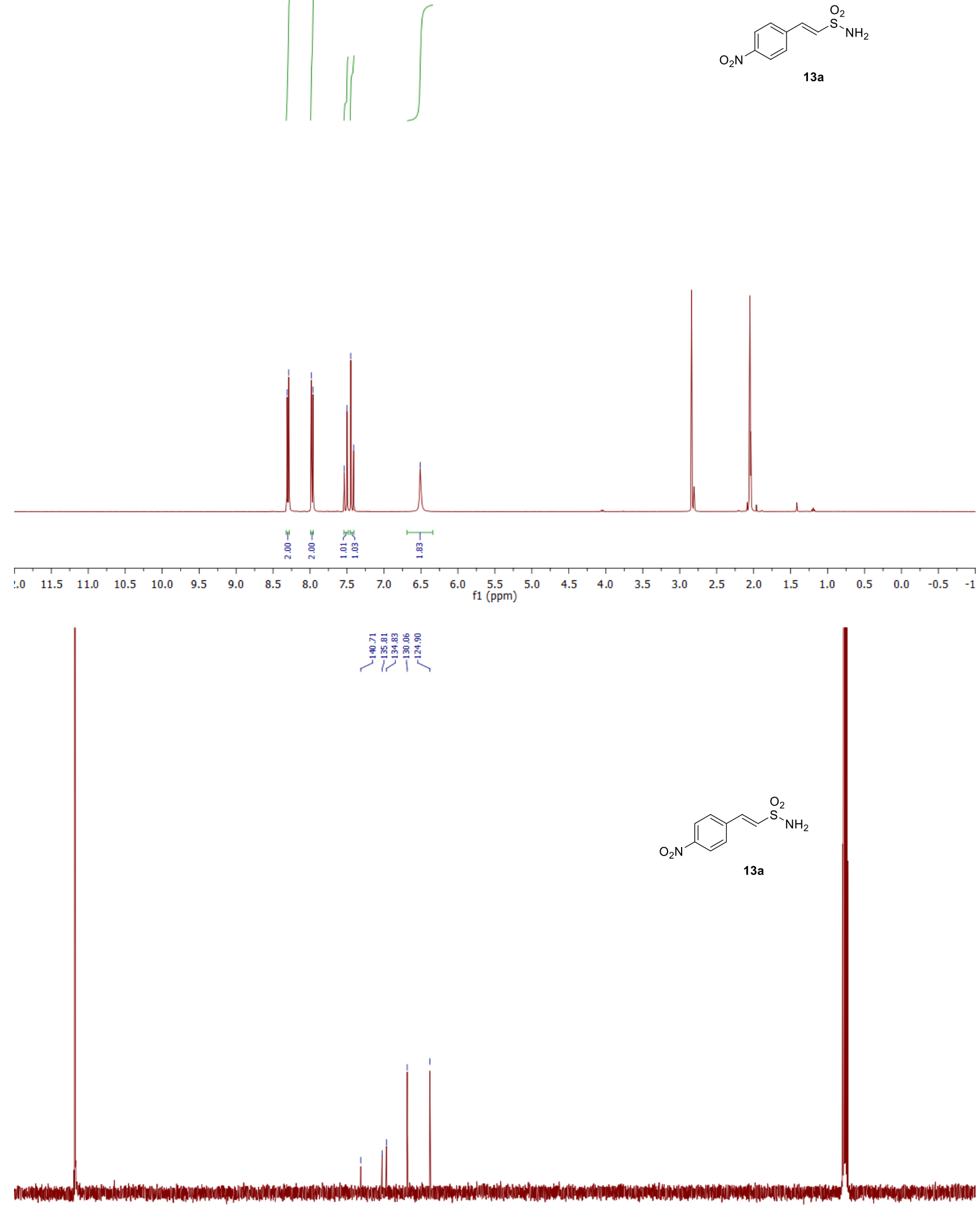

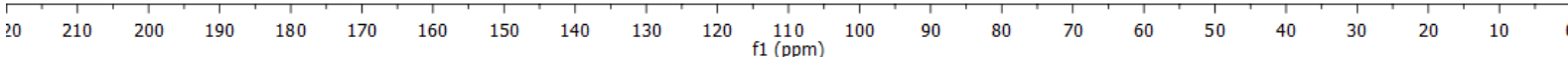




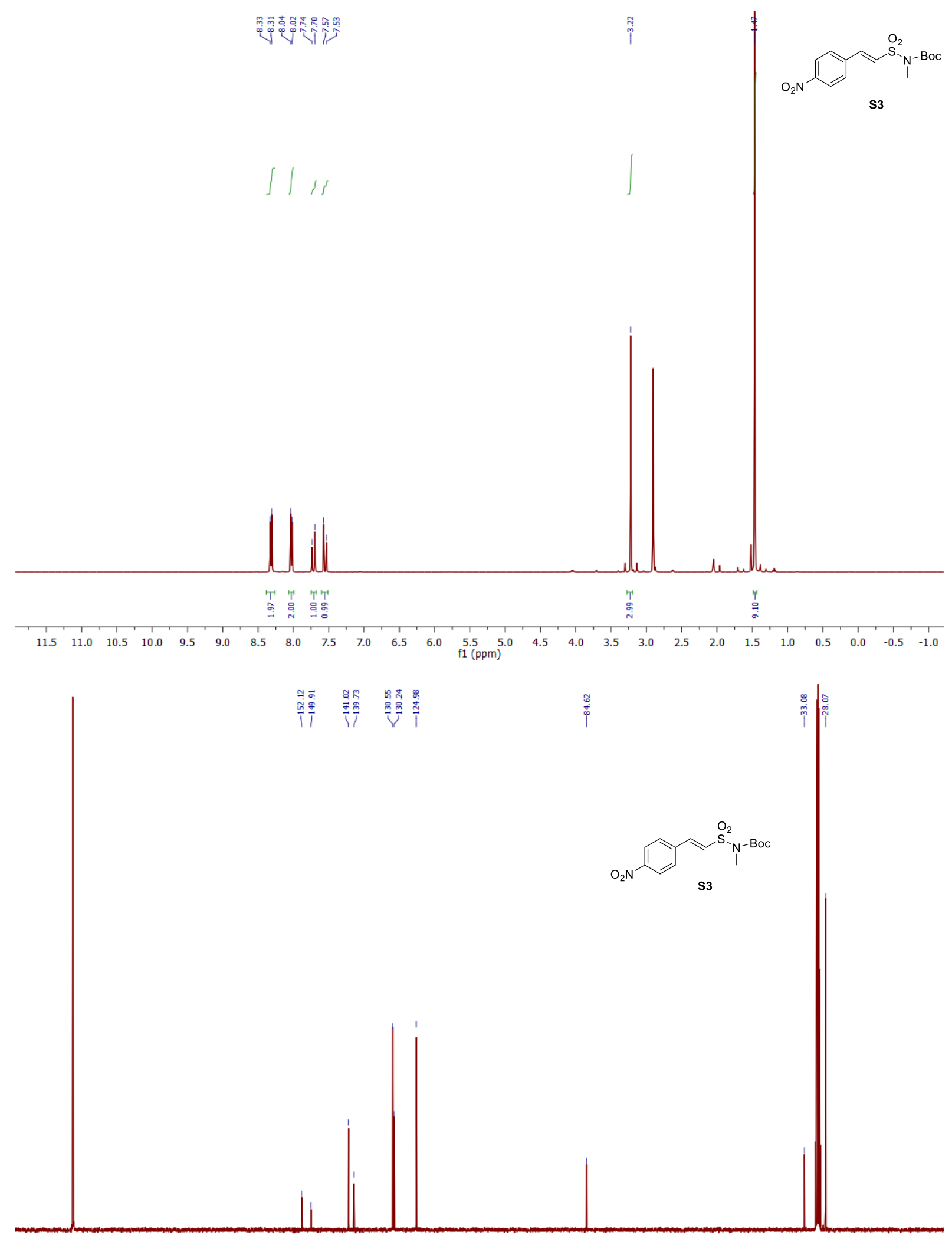

\begin{tabular}{lllllllllllllllllllllllll}
\hline 20 & 210 & 200 & 190 & 180 & 170 & 160 & 150 & 140 & 130 & 120 & 110 & 100 & 90 & 80 & 70 & 60 & 50 & 40 & 30 & 20 & 10 & $\mathrm{C}$
\end{tabular} 

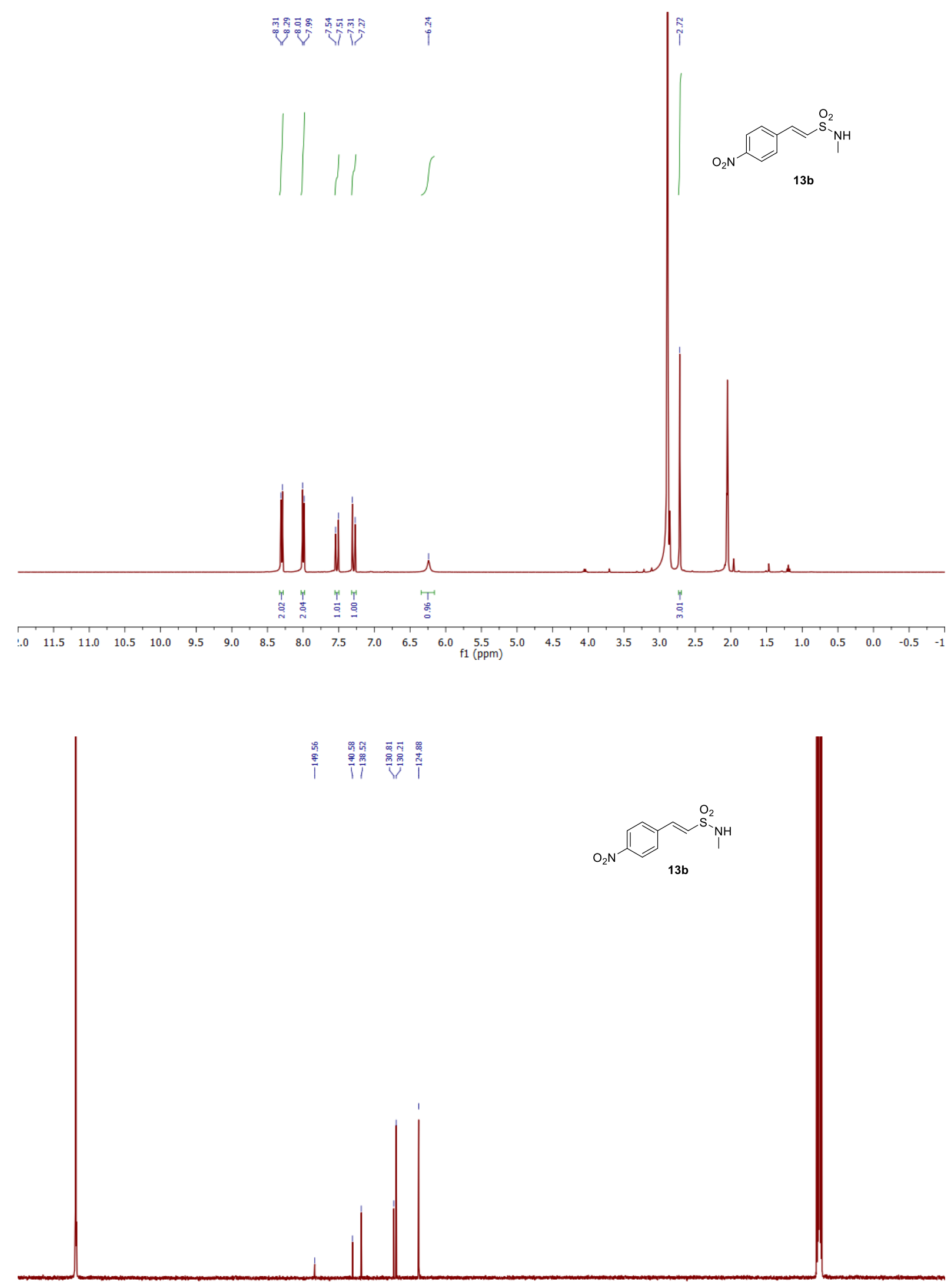

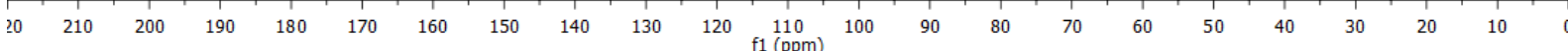


$8 a$

$400 \mathrm{MHz}, \mathrm{CDCl} 3$

$\underbrace{\infty}$

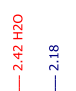
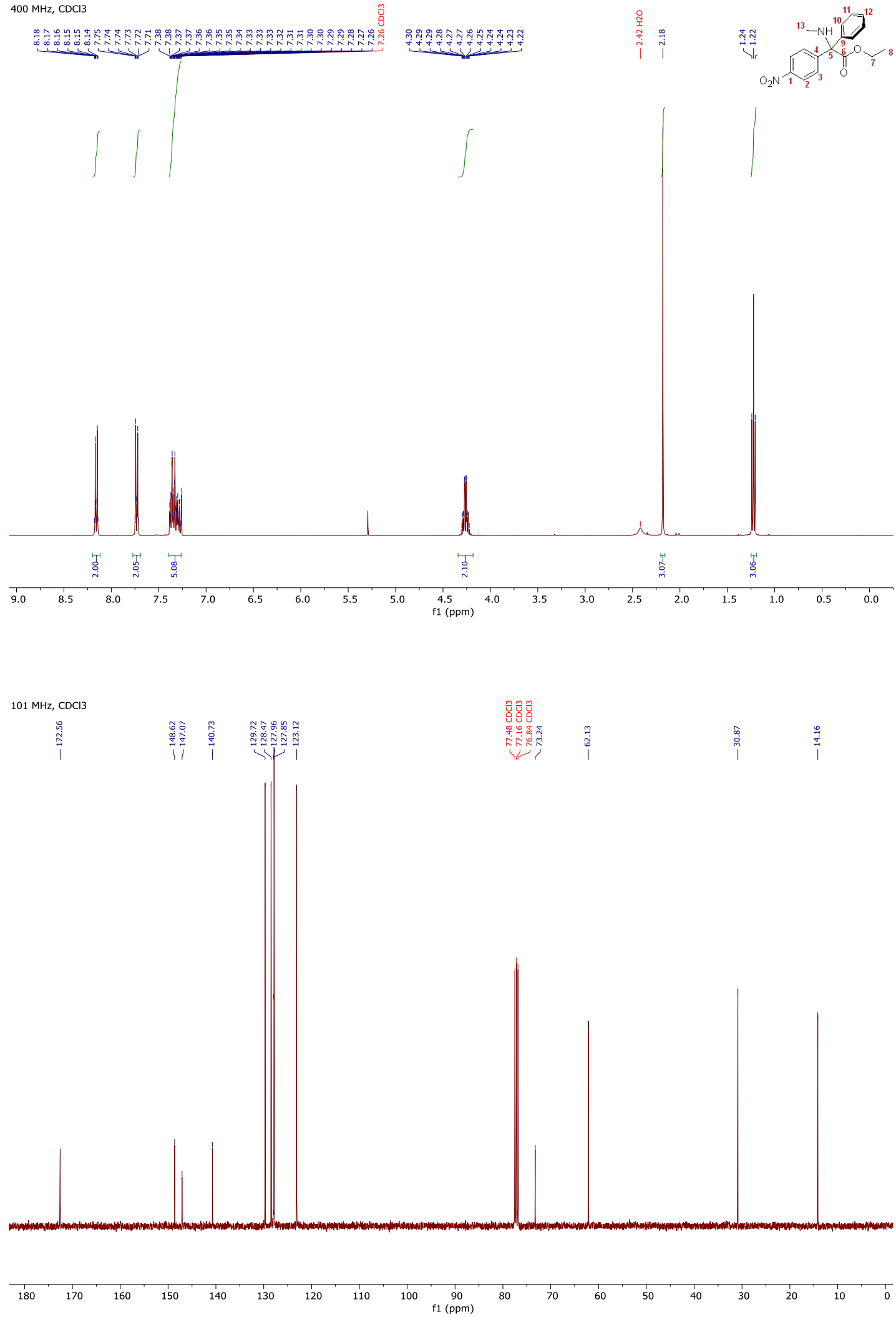

S39 
$8 b$

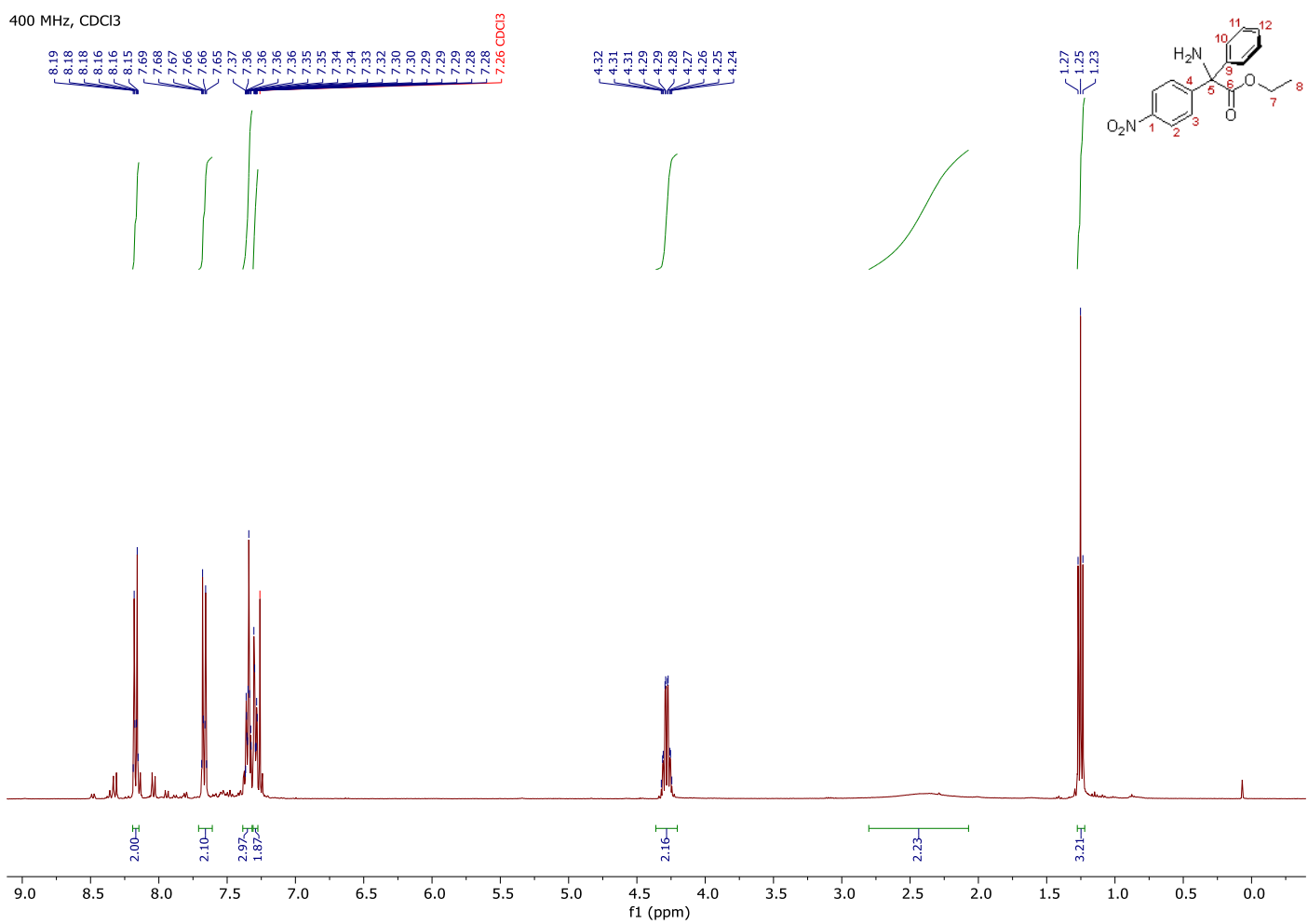

$101 \mathrm{MHz}, \mathrm{CDCl} 3$

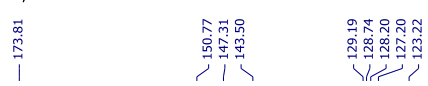
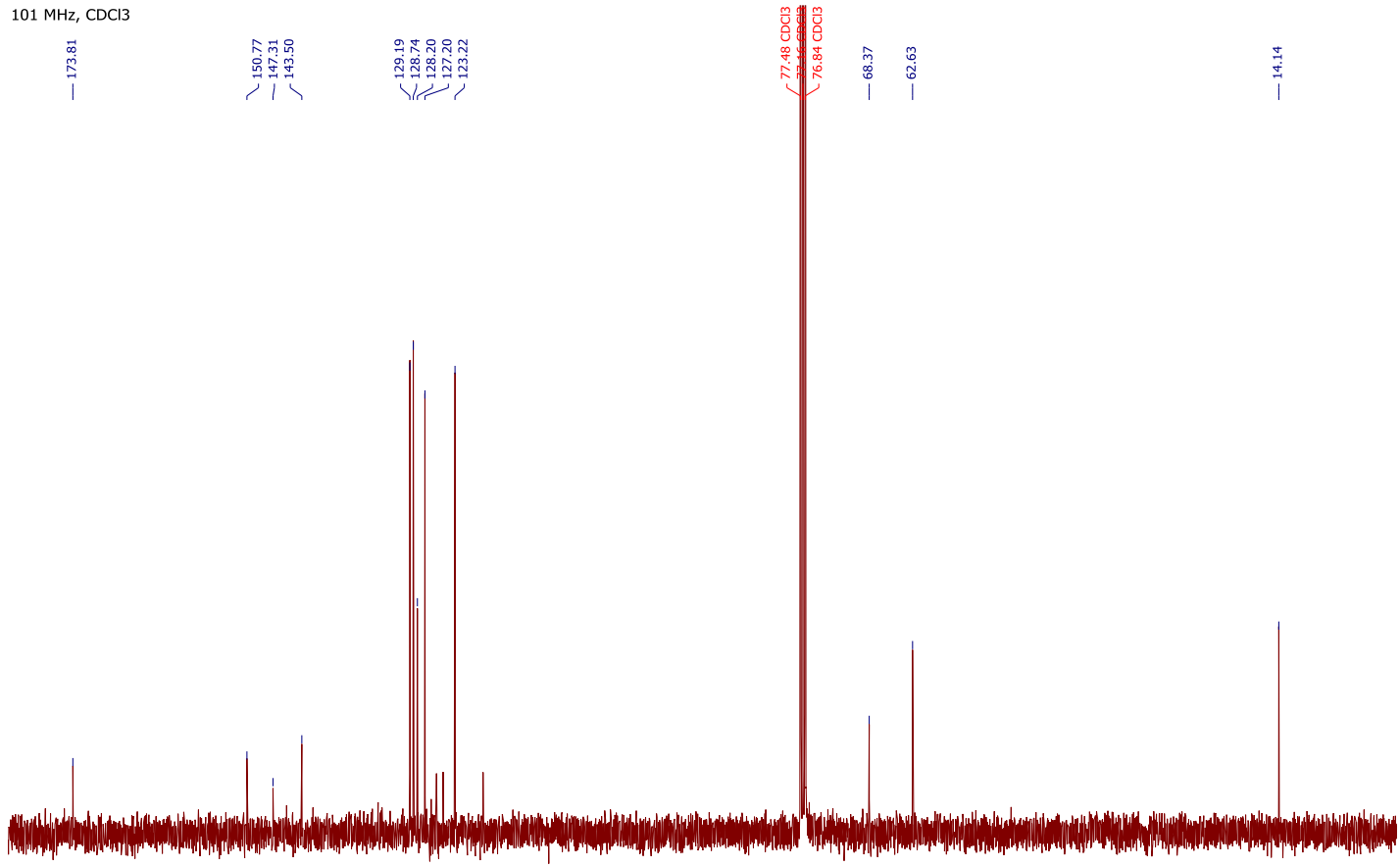

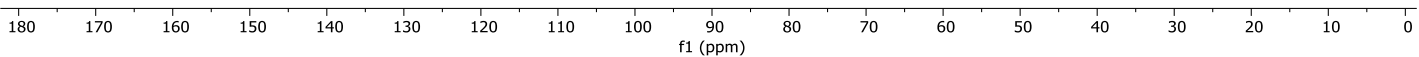


$8 c$

$400 \mathrm{MHz}, \mathrm{CDCl} 3$
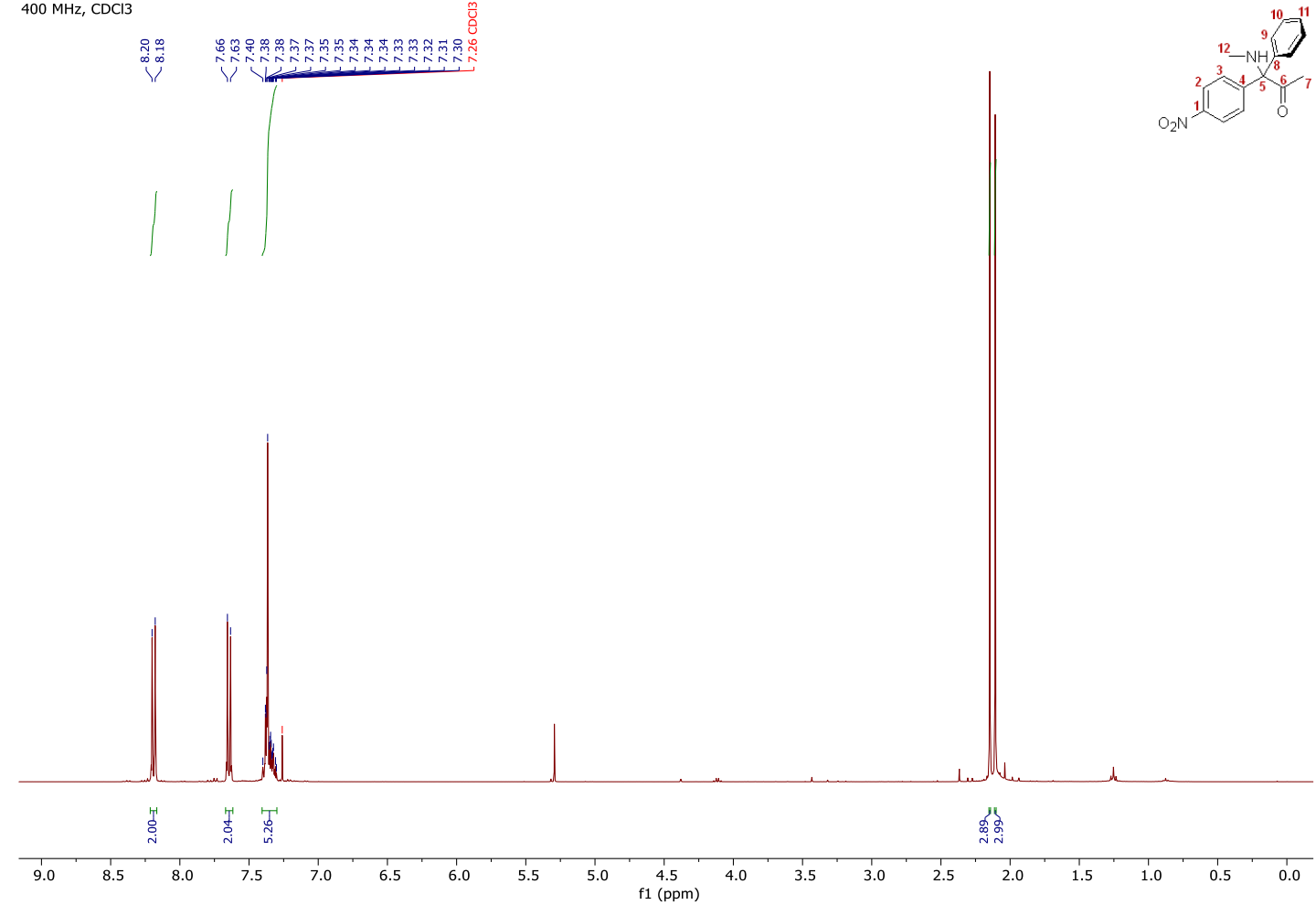

$101 \mathrm{MHz}, \mathrm{CDCl} 3$

总

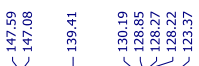

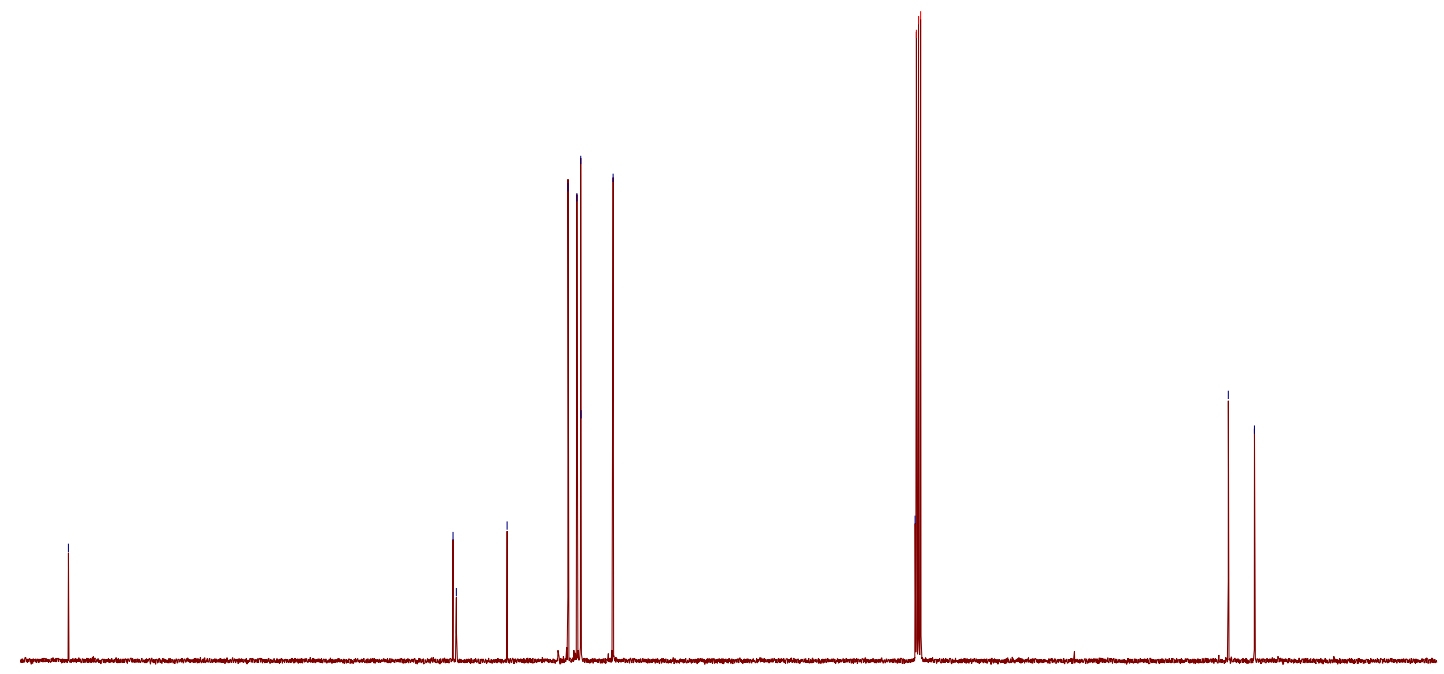

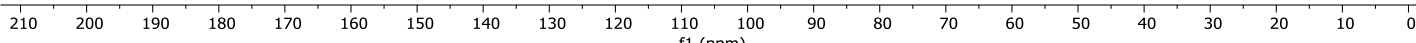


$8 d$
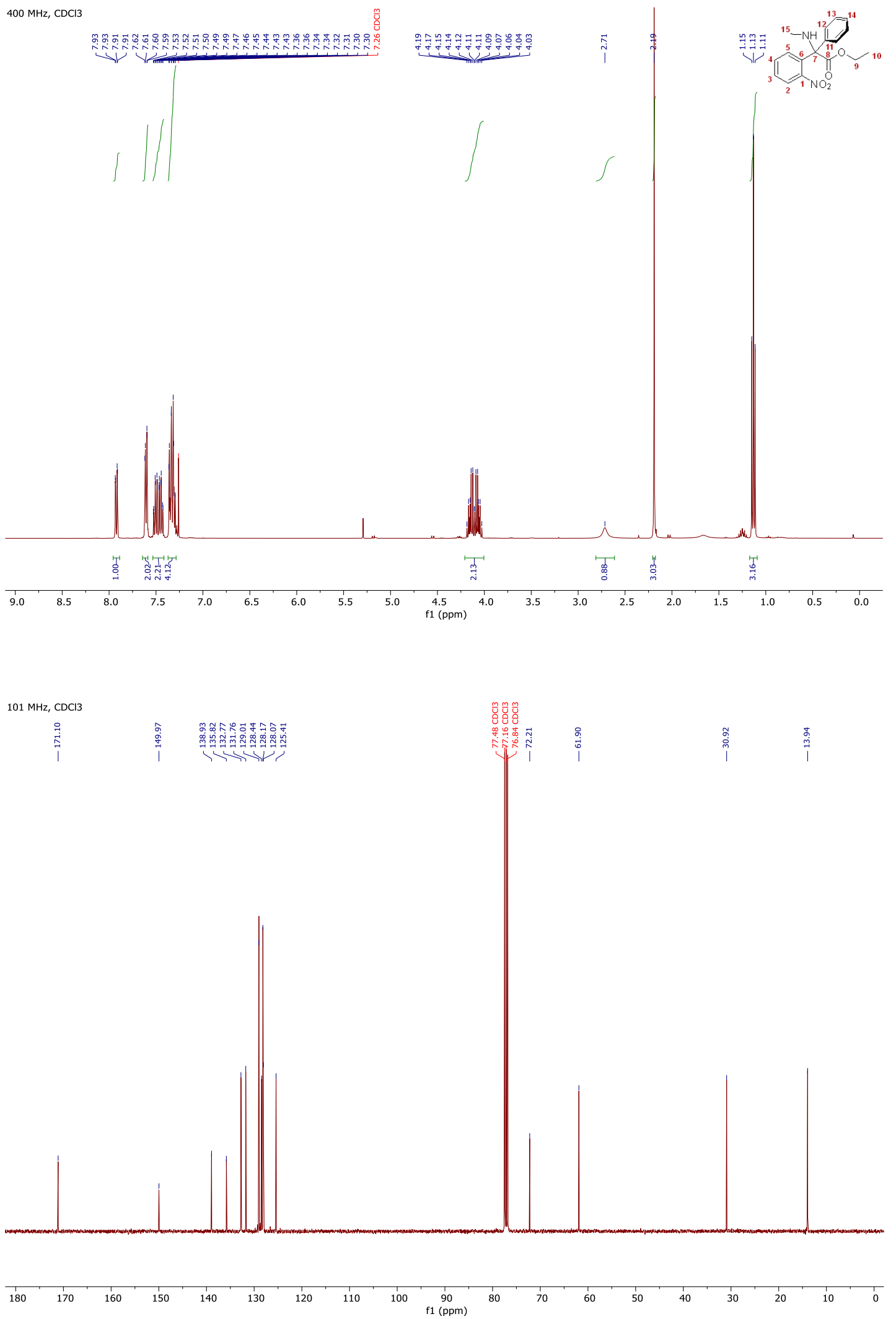
$8 e$
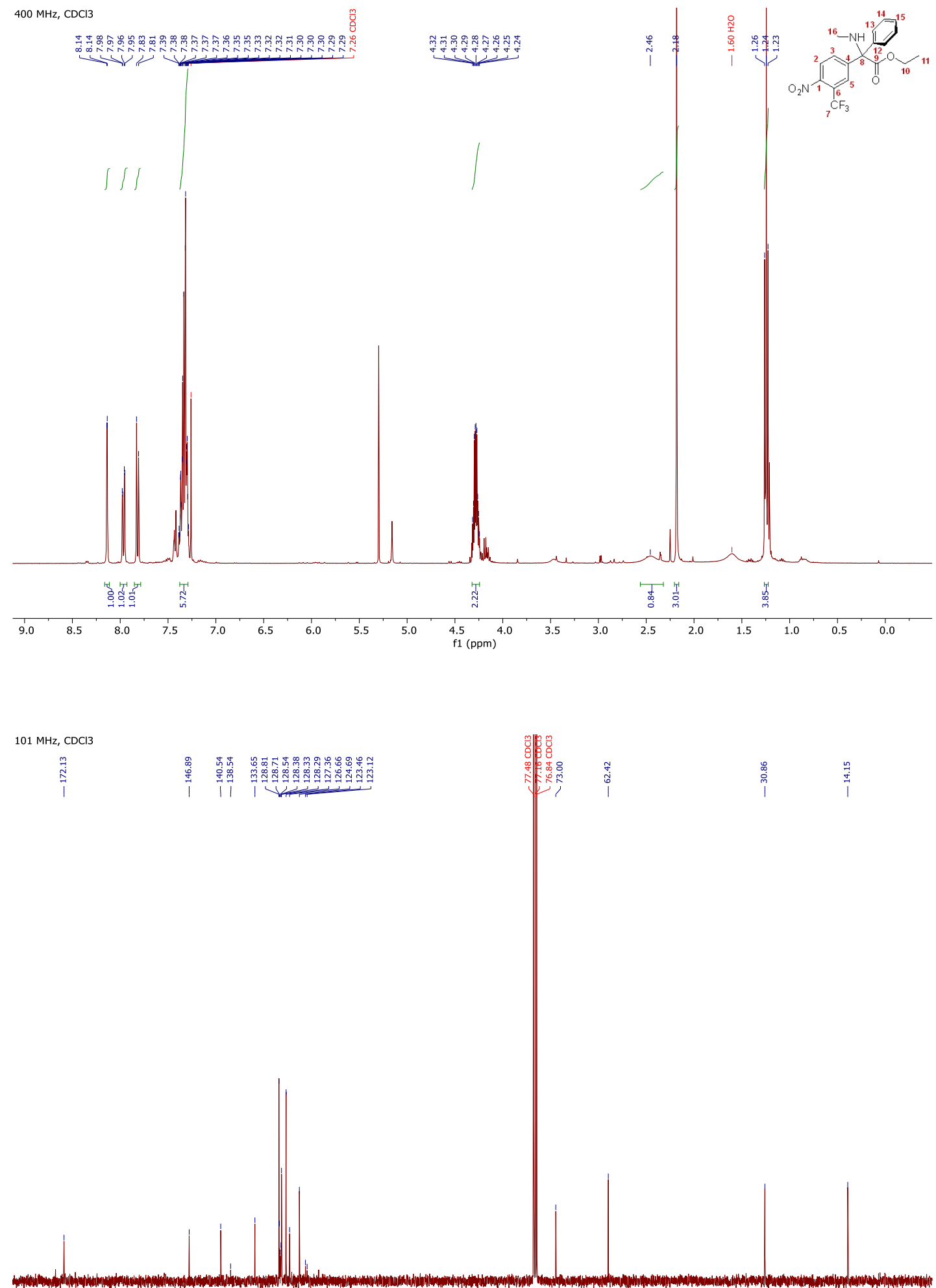

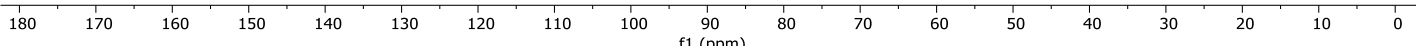


$376 \mathrm{MHz}$, CDCl3

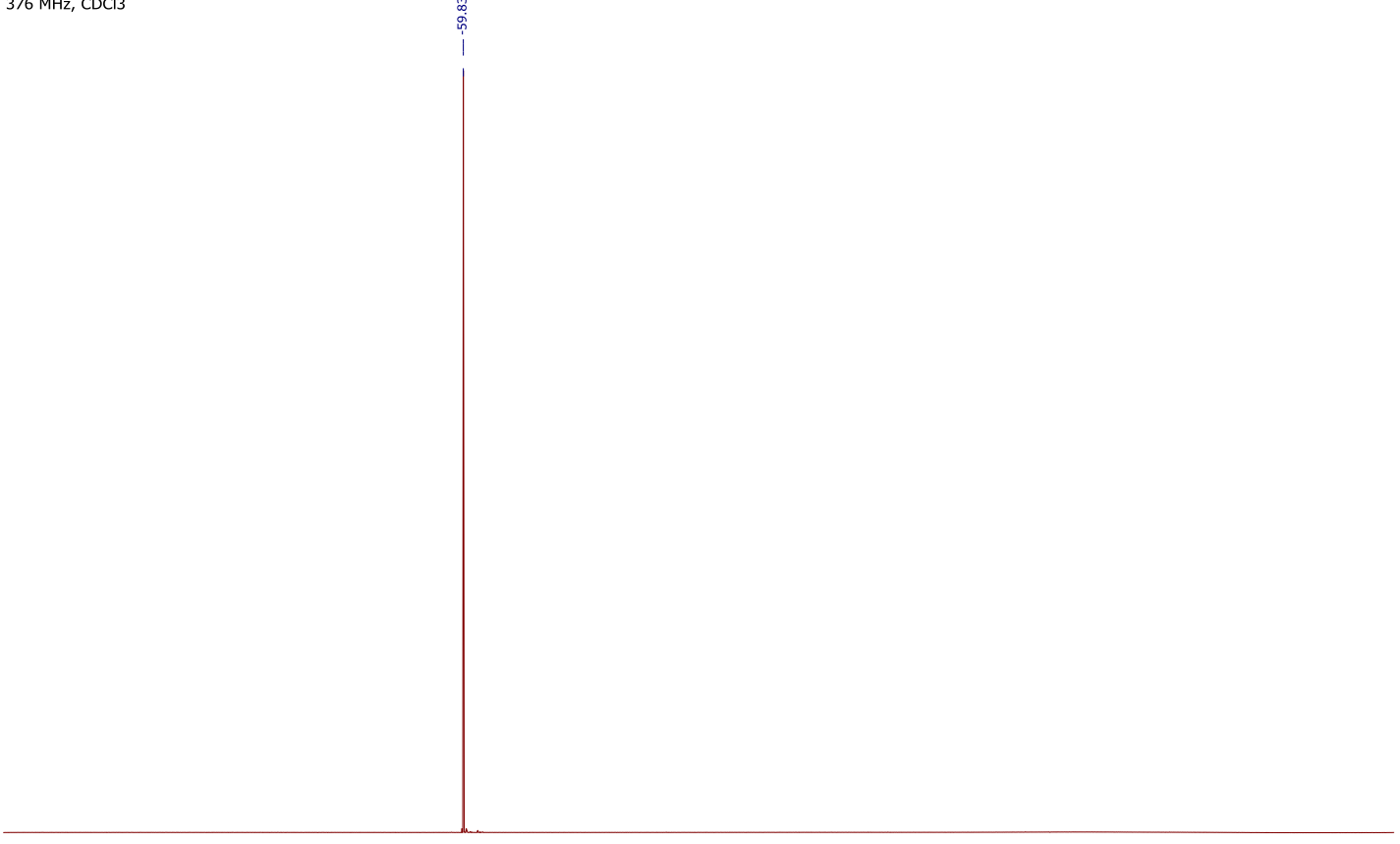

$\begin{array}{lllllllllllllllllllllllllll}10 & 0 & -10 & -20 & -30 & -40 & -50 & -60 & -70 & -80 & -90 & -100 & -110 & -120 & -130 & -140 & -150 & -160 & -170 & -180 & -190 & -200 & -210\end{array}$ 
$400 \mathrm{MHz}, \mathrm{CDCl} 3$

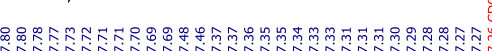
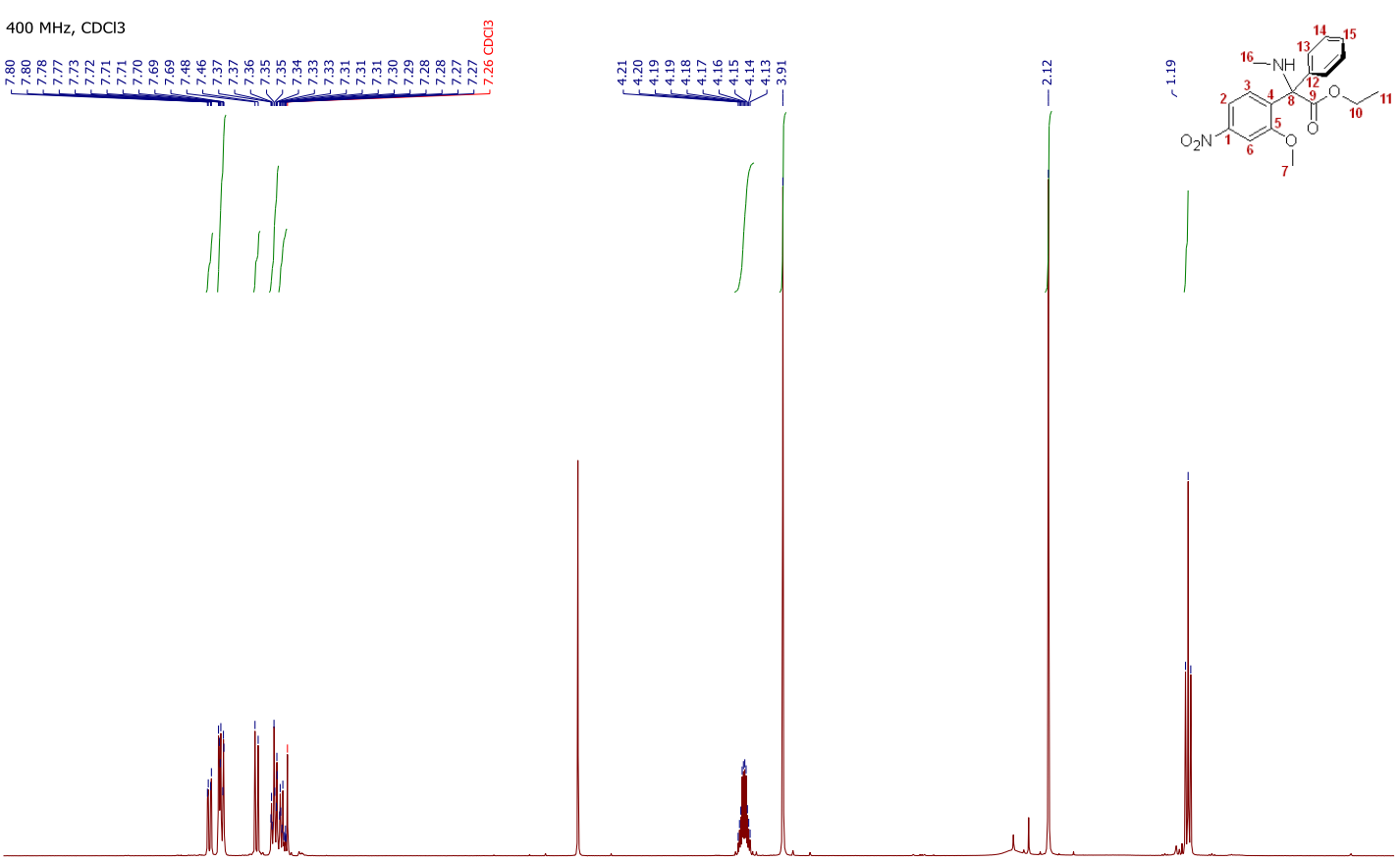

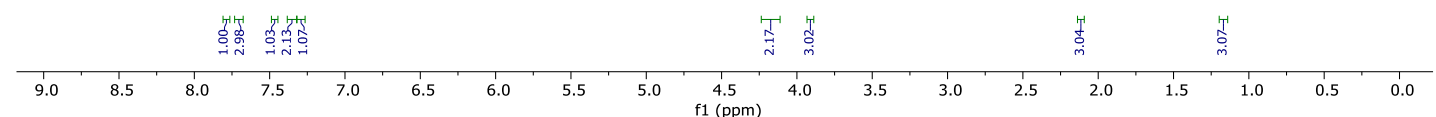

$101 \mathrm{MHz}, \mathrm{CDCl} 3$

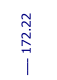

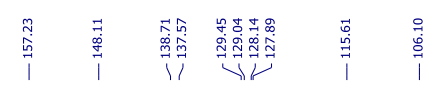

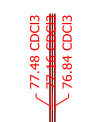

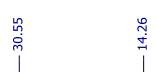

180

$\frac{1}{150} \quad 140$

130

90
$\mathrm{f} 1(\mathrm{ppm})$

$80 \quad 70$

60

40

$3 0 \longdiv { 2 0 } 1 0 \quad 1$ 
$400 \mathrm{MHz}, \mathrm{CDCl} 3$
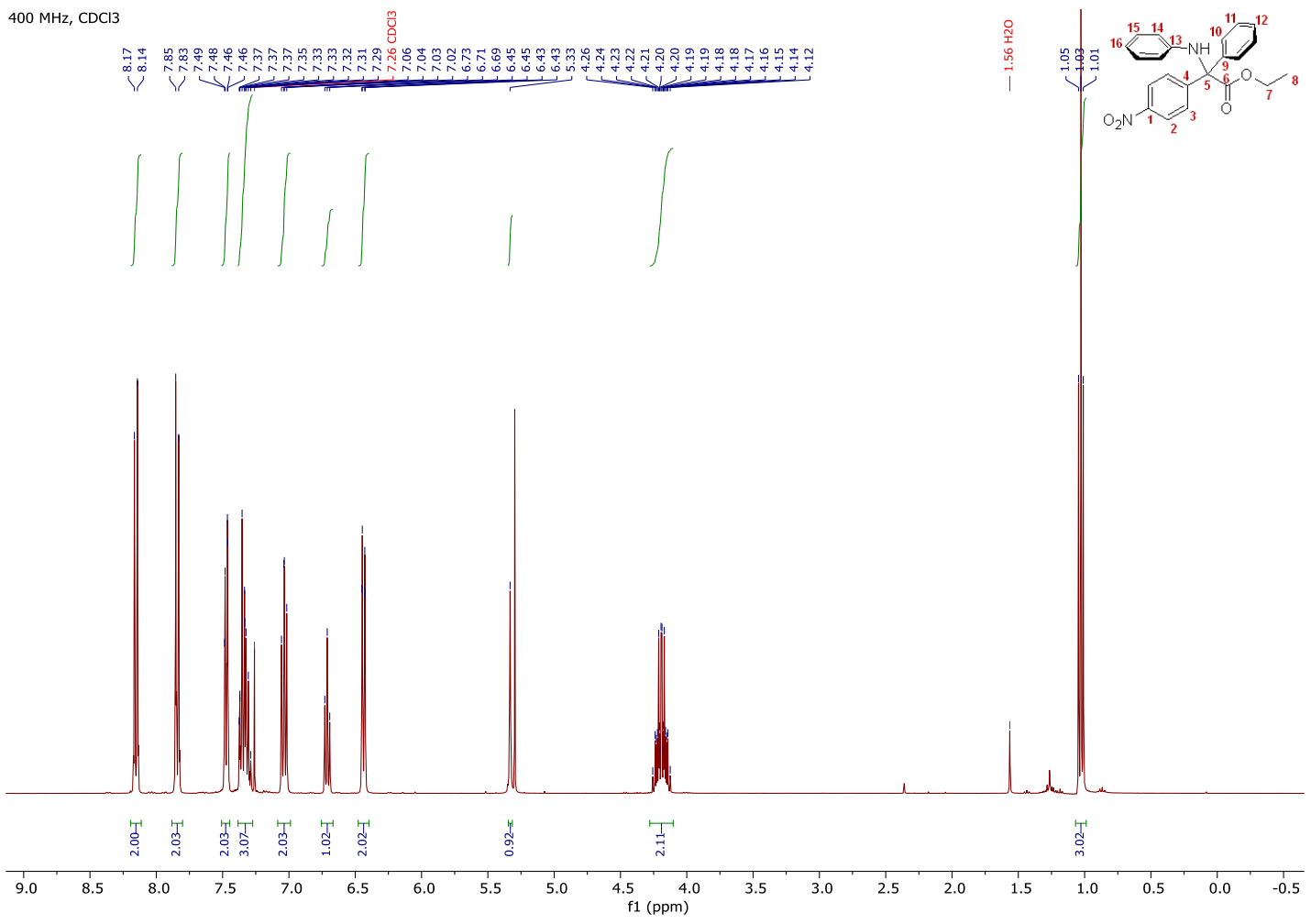

$101 \mathrm{MHz}, \mathrm{CDCl} 3$

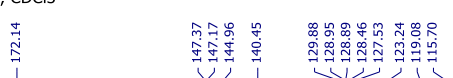

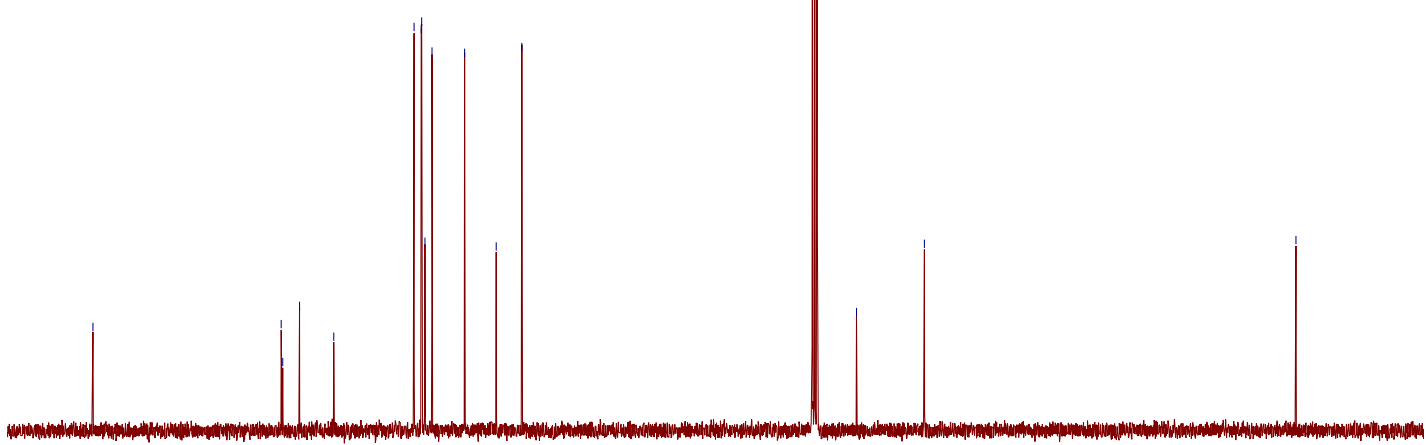

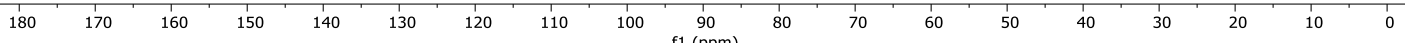


$8 \mathrm{~h}$

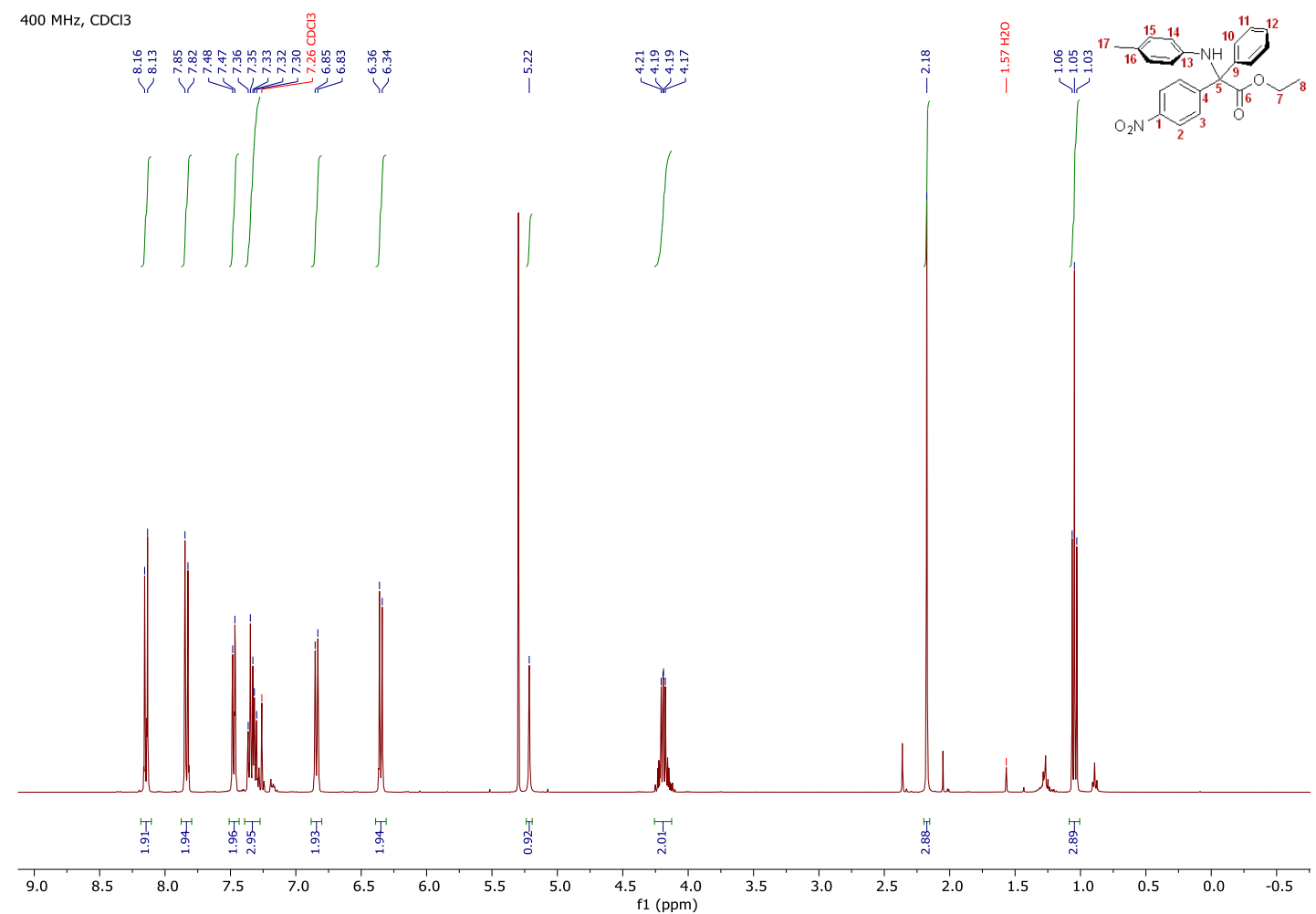

$101 \mathrm{MHz}, \mathrm{CDCl} 3$

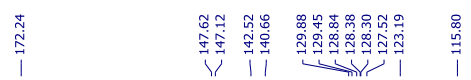
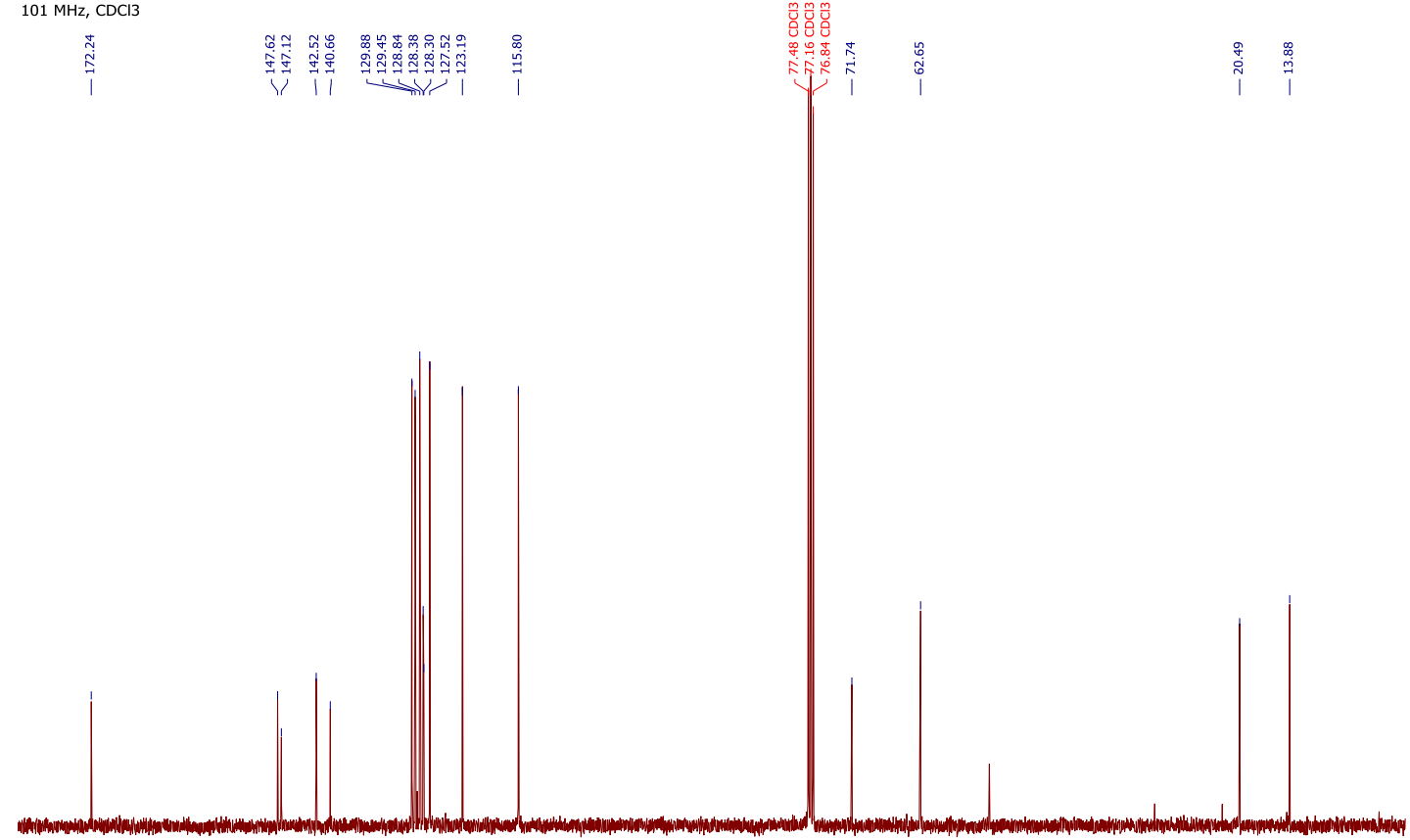

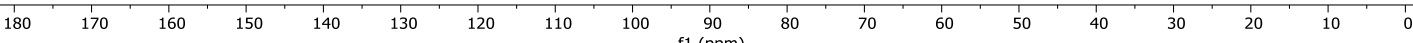


$8 i$
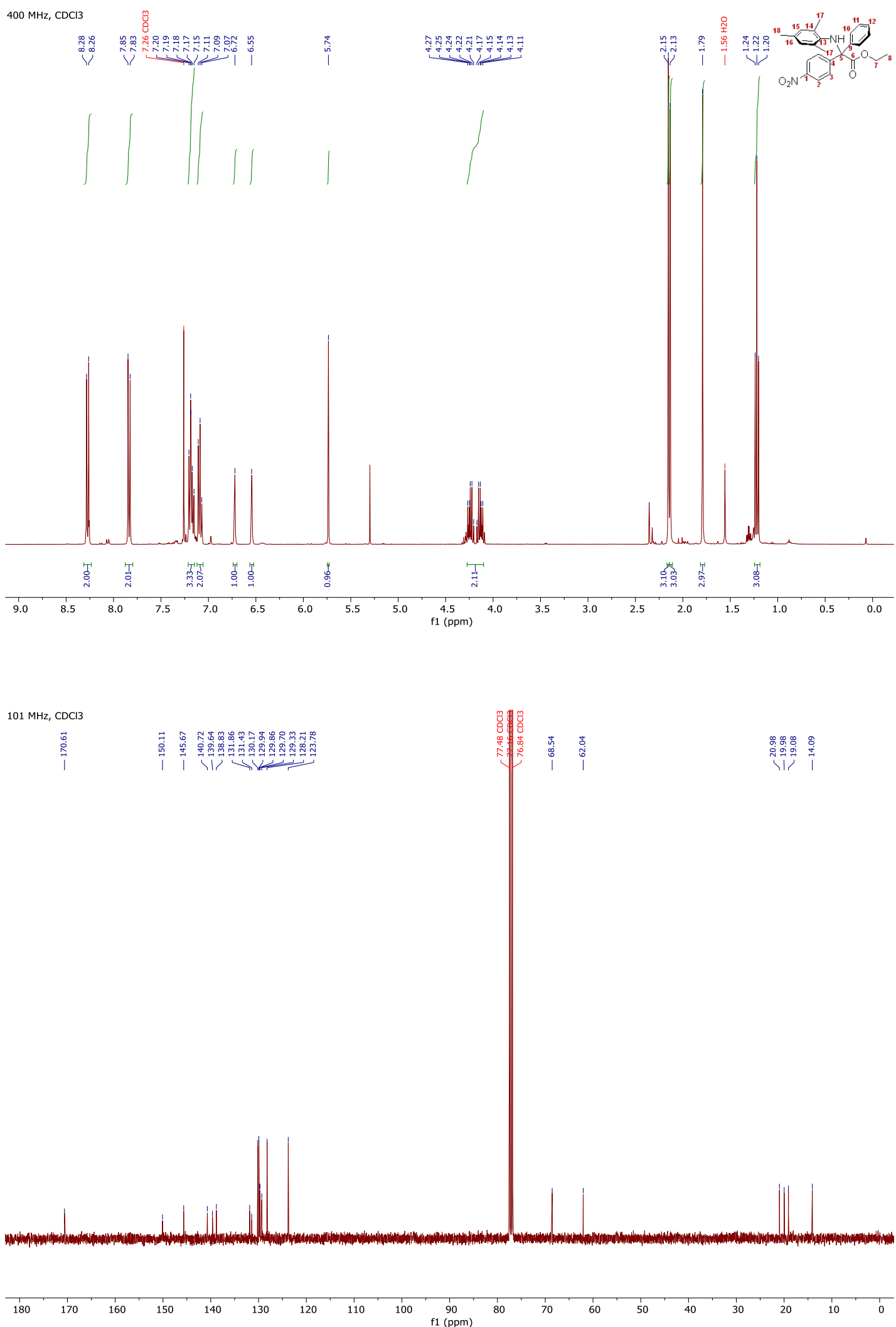


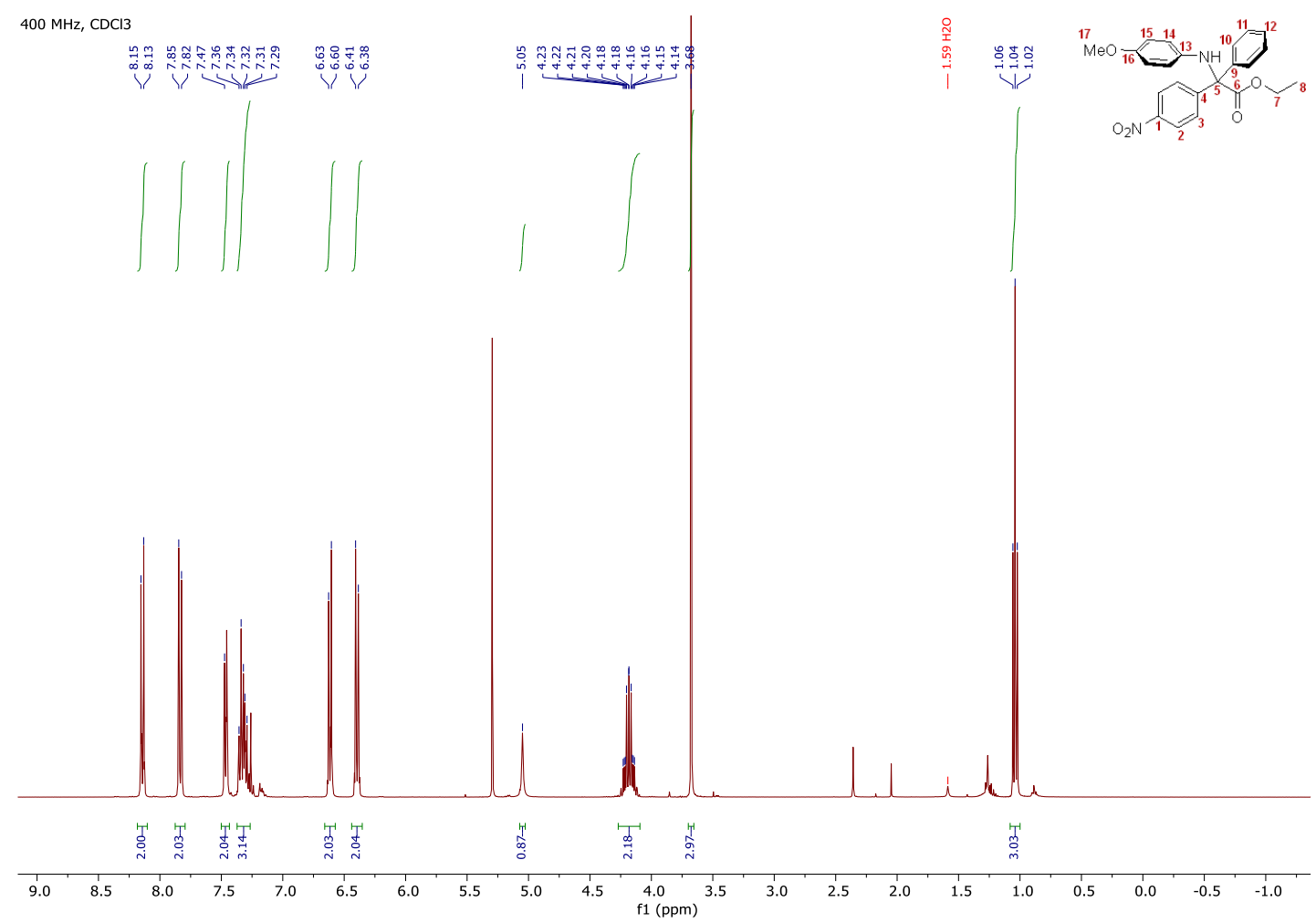

$101 \mathrm{MHz}, \mathrm{CDCl} 3$

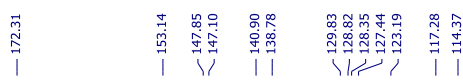

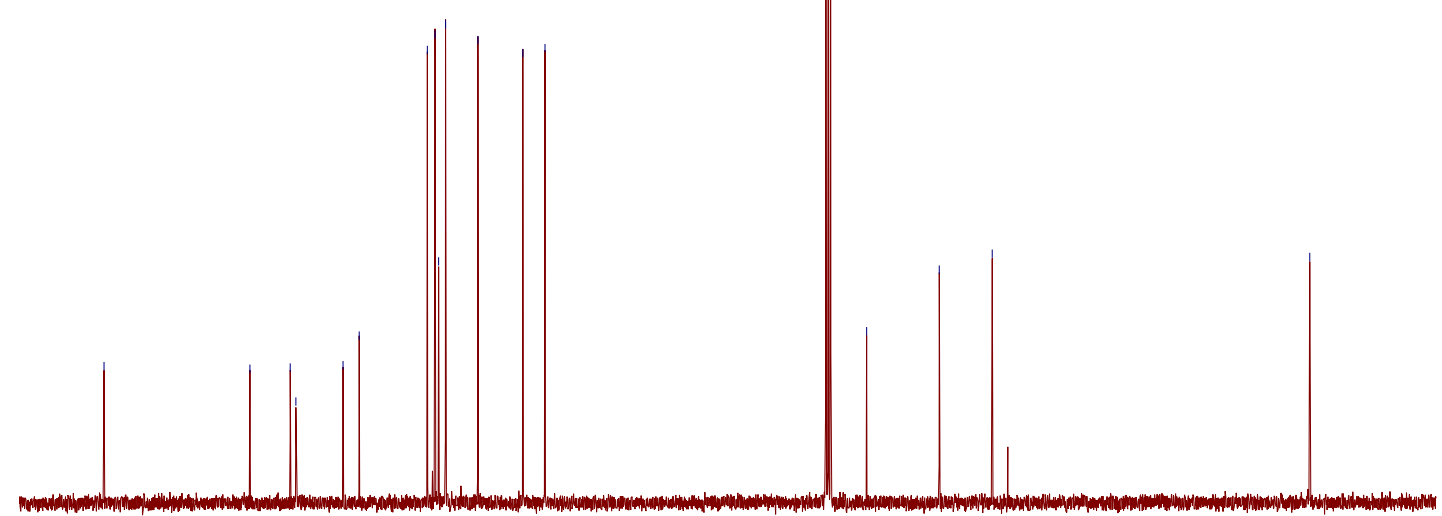

$180 \quad 170 \quad 160$

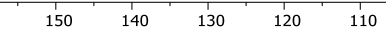

10090

70

50 

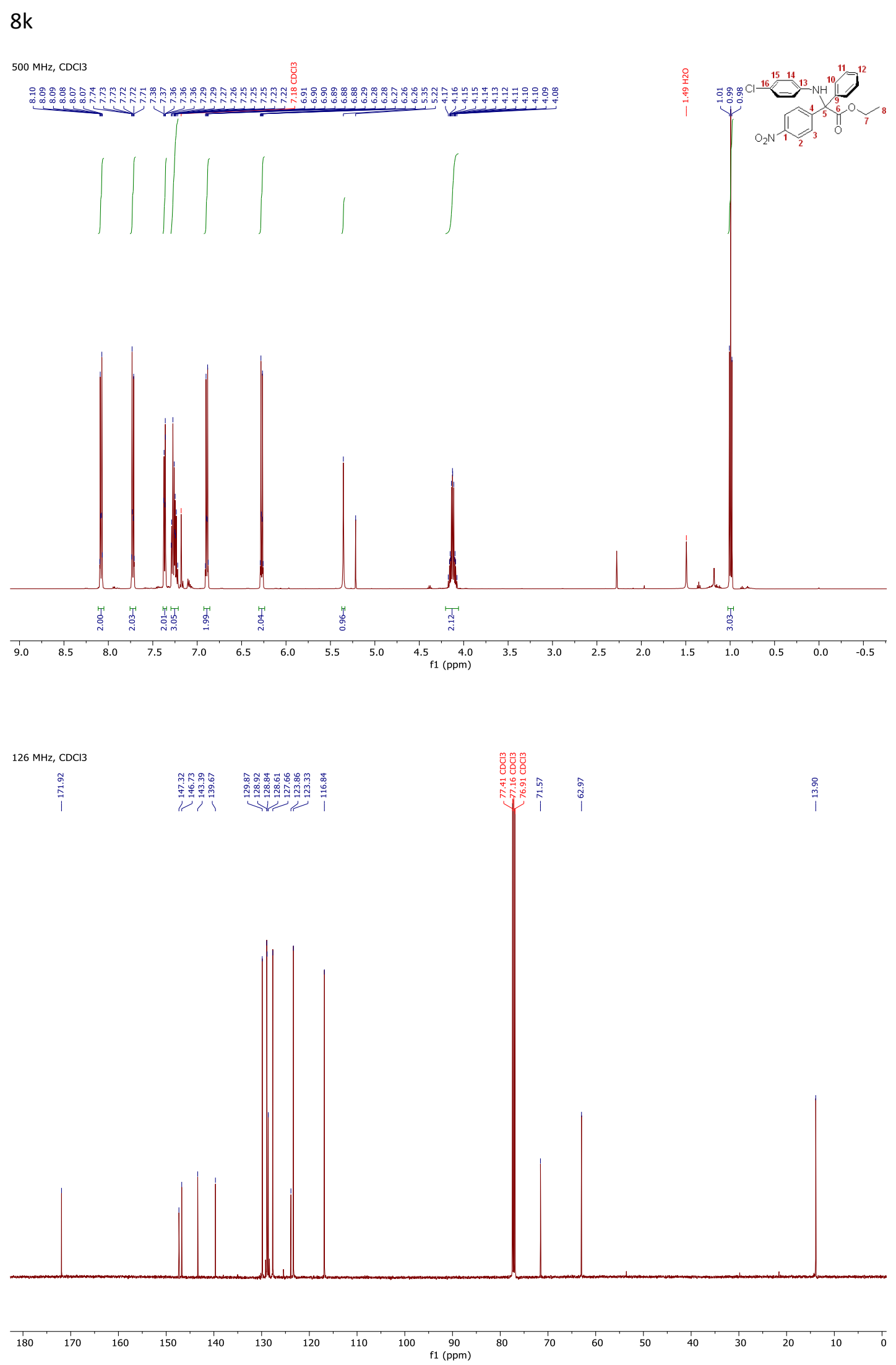

S50 
$400 \mathrm{MHz}, \mathrm{CDCl} 3$

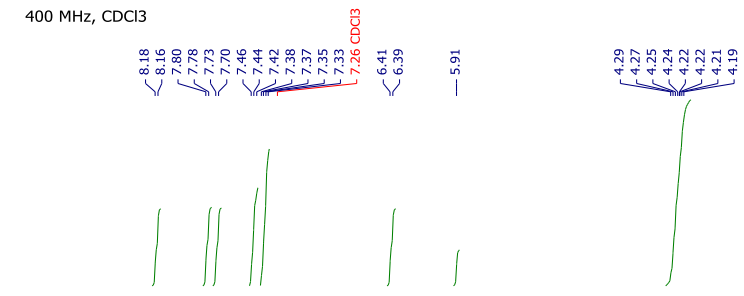

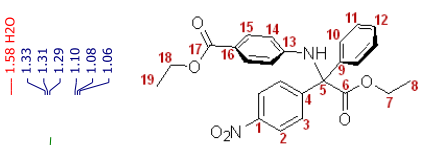

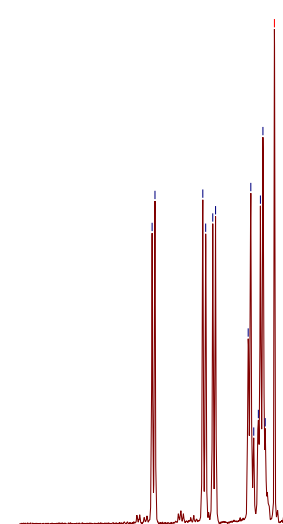

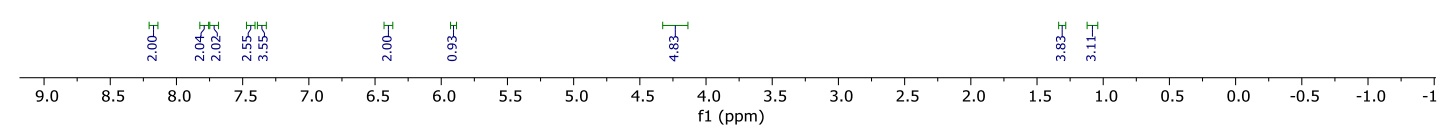

$101 \mathrm{MHz}, \mathrm{CDCl} 3$

\begin{tabular}{|c|c|c|}
\hline 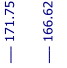 & 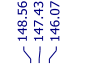 & \\
\hline
\end{tabular}

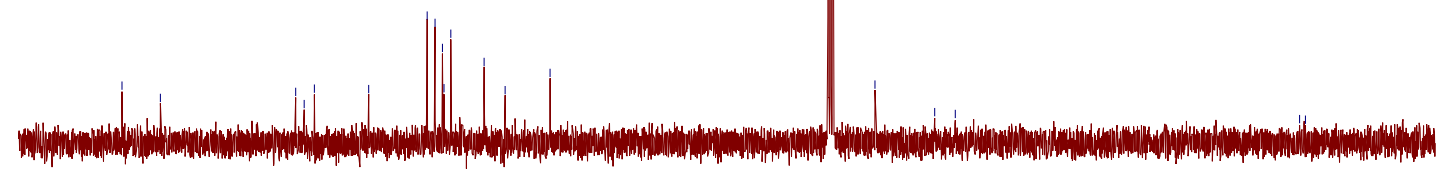


$8 m$
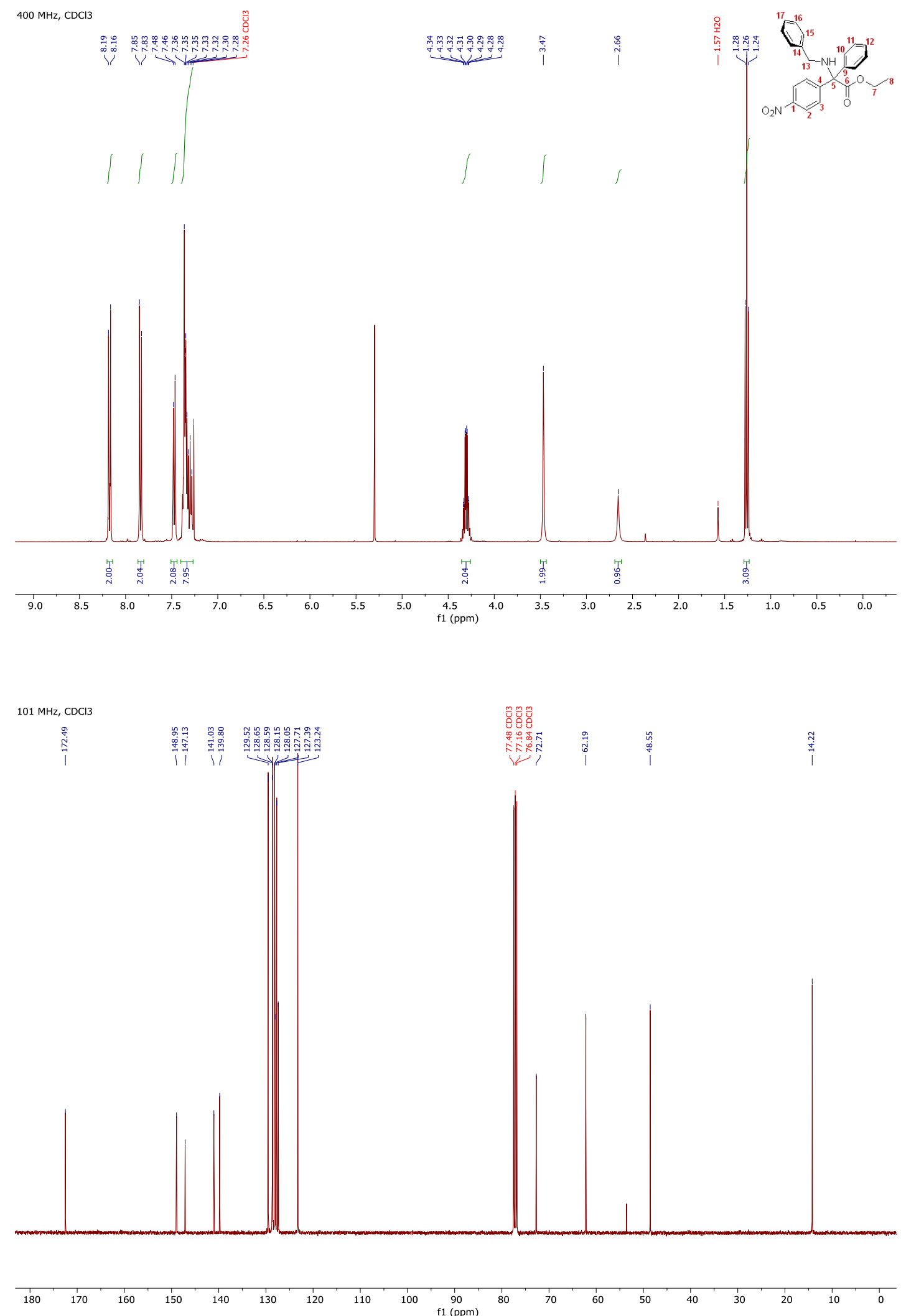
$8 n$

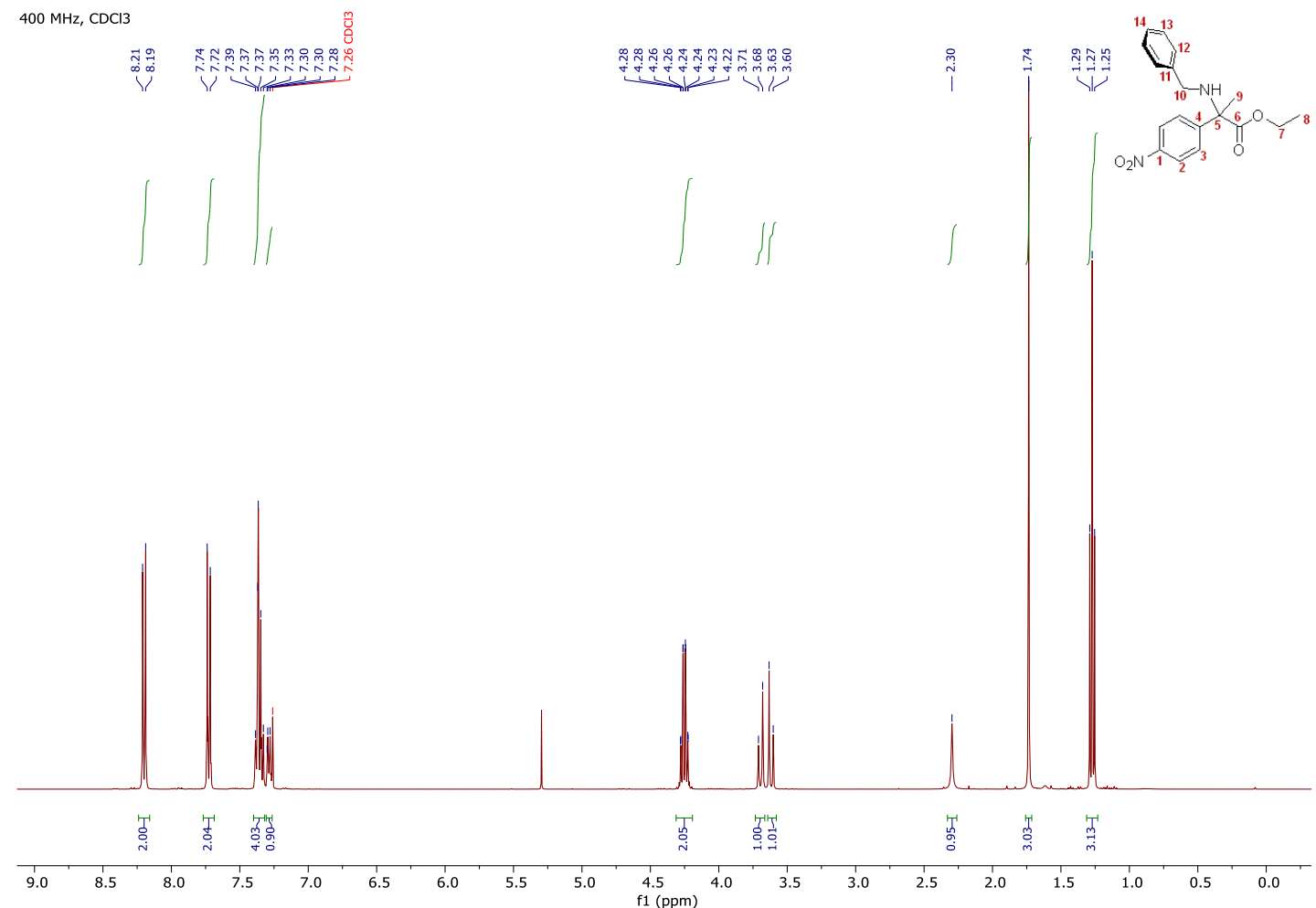

$101 \mathrm{MHz}, \mathrm{CDCl} 3$

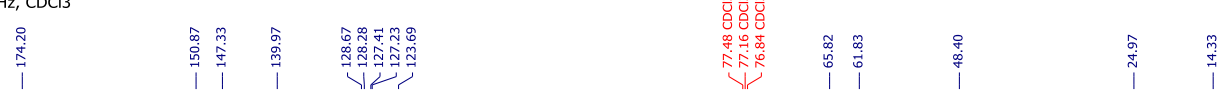

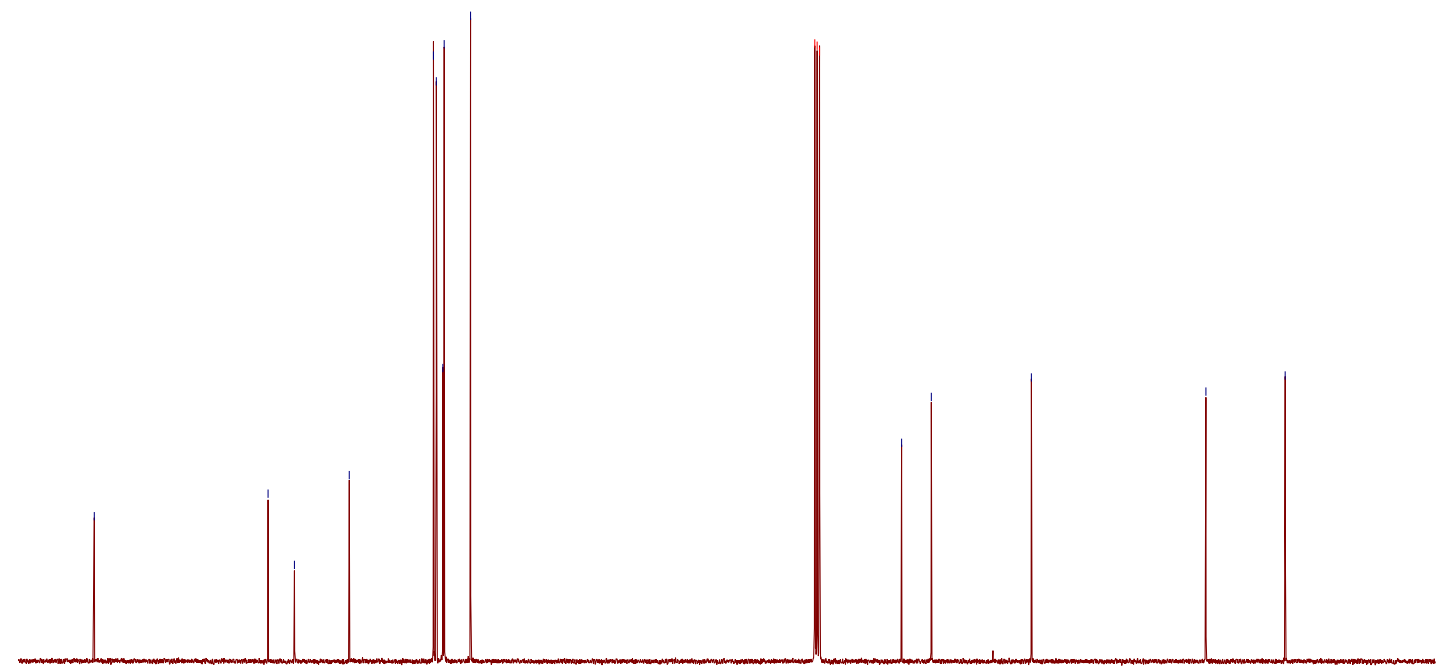

$\begin{array}{llllllllll}180 & 170 & 160 & 150 & 140 & 130 & 120 & 110 & 100 & \begin{array}{c}1 \\ \mathrm{f}(\mathrm{ppm})\end{array}\end{array}$ 
80

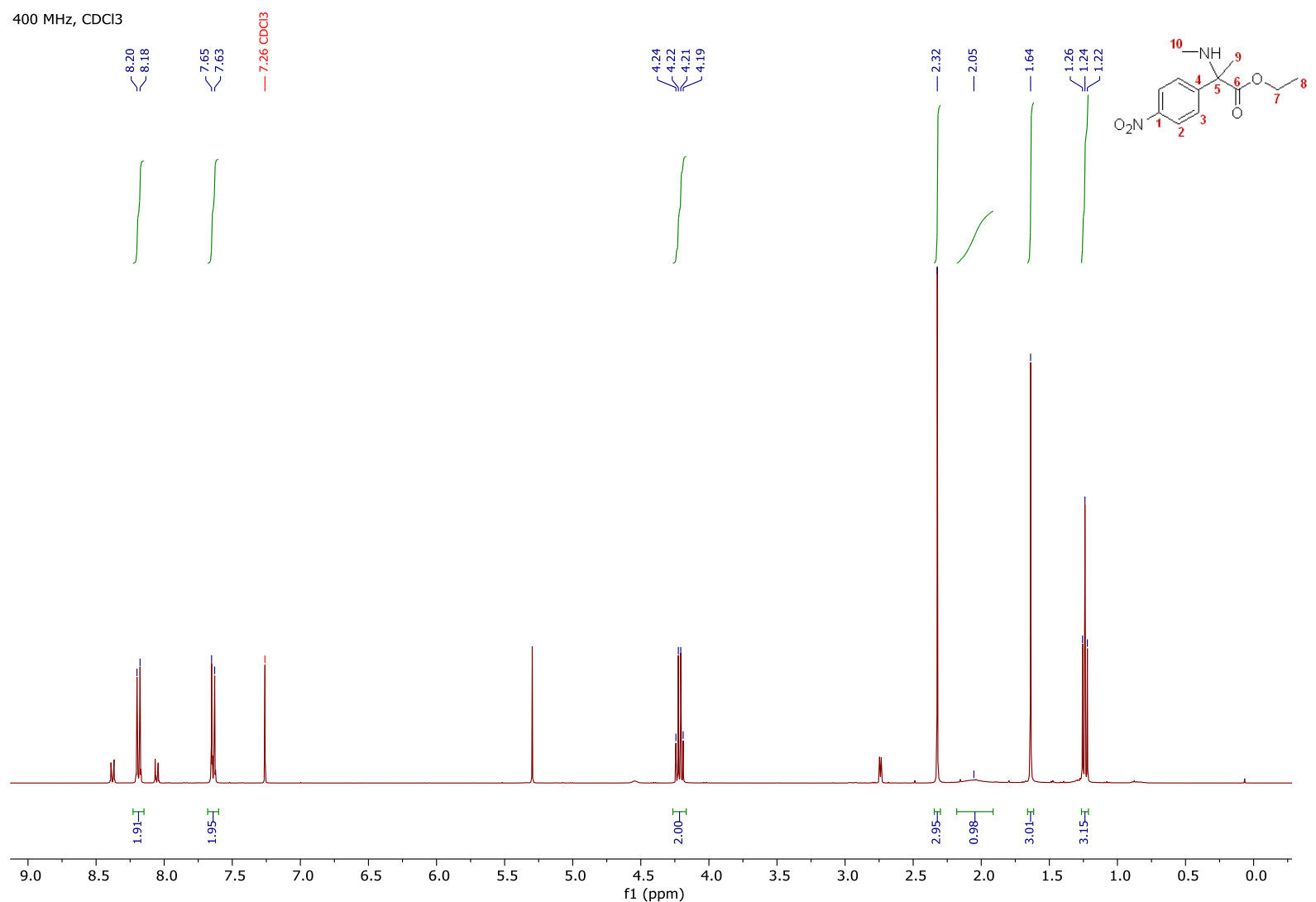

$101 \mathrm{MHz}, \mathrm{CDCl} 3$

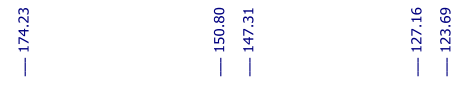

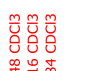

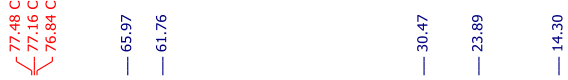

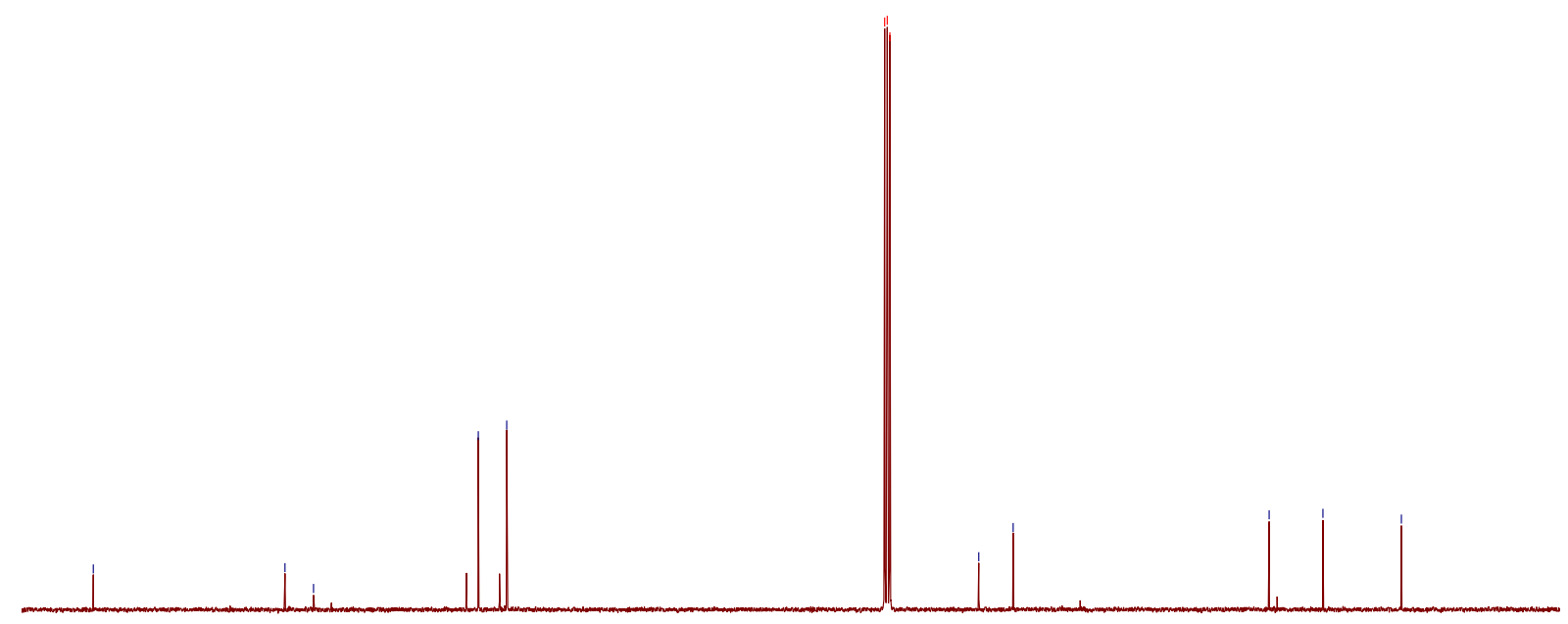

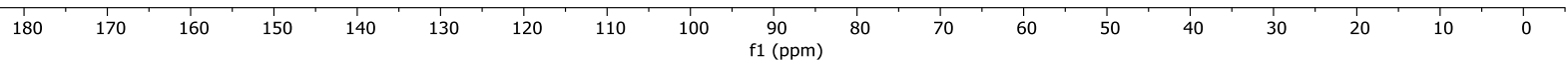


$8 p$
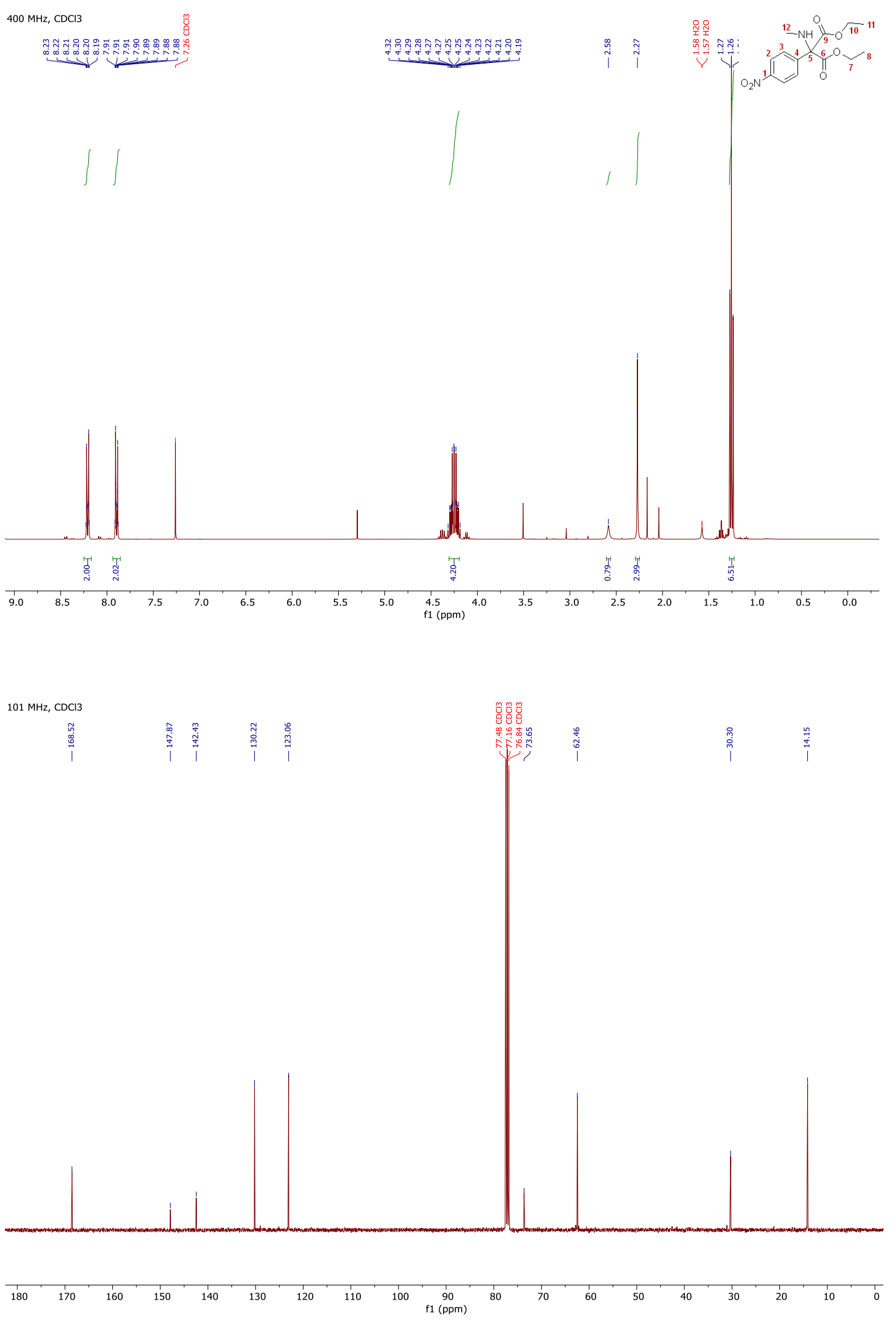

S55 
$8 q$
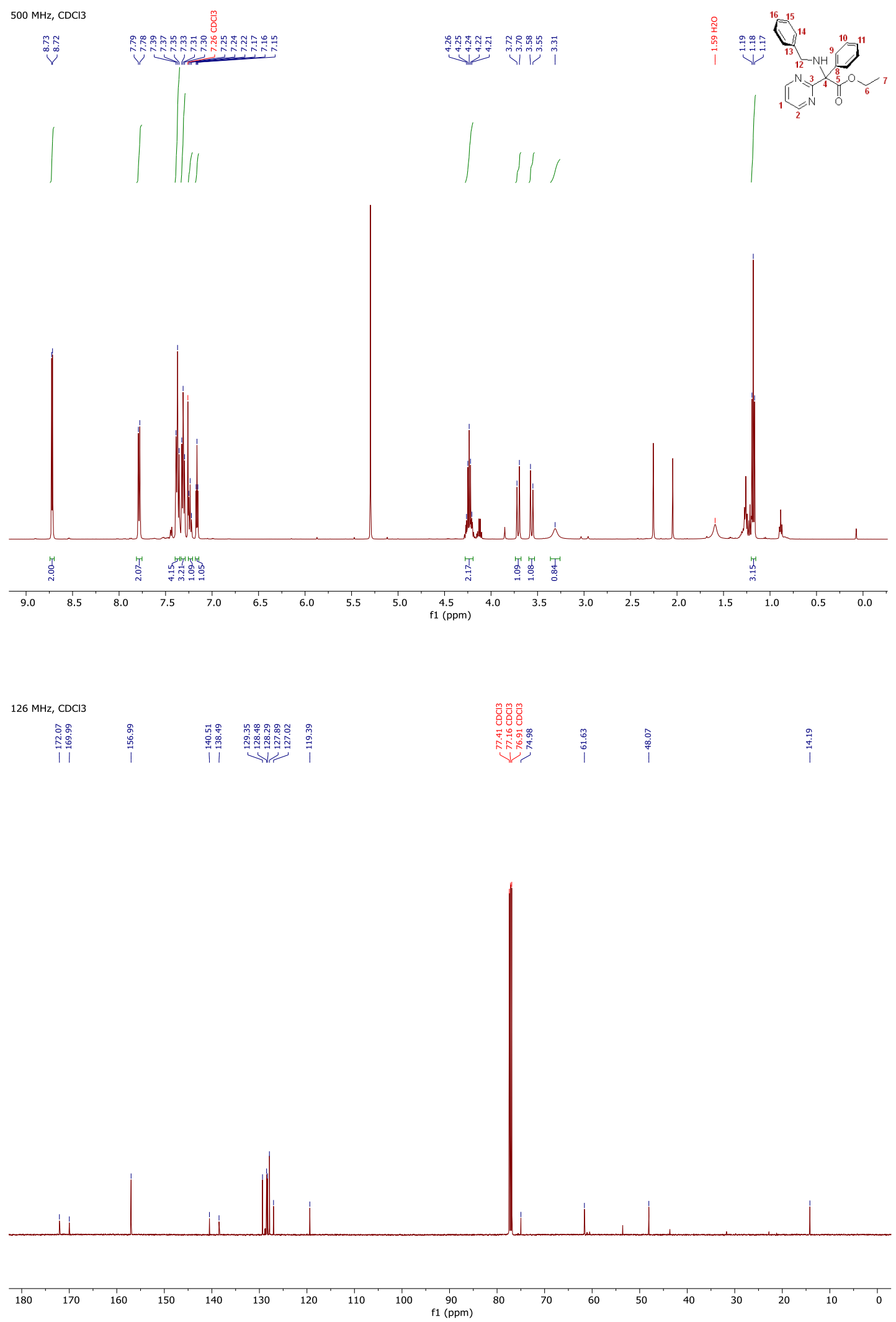

S56 
$11 a$

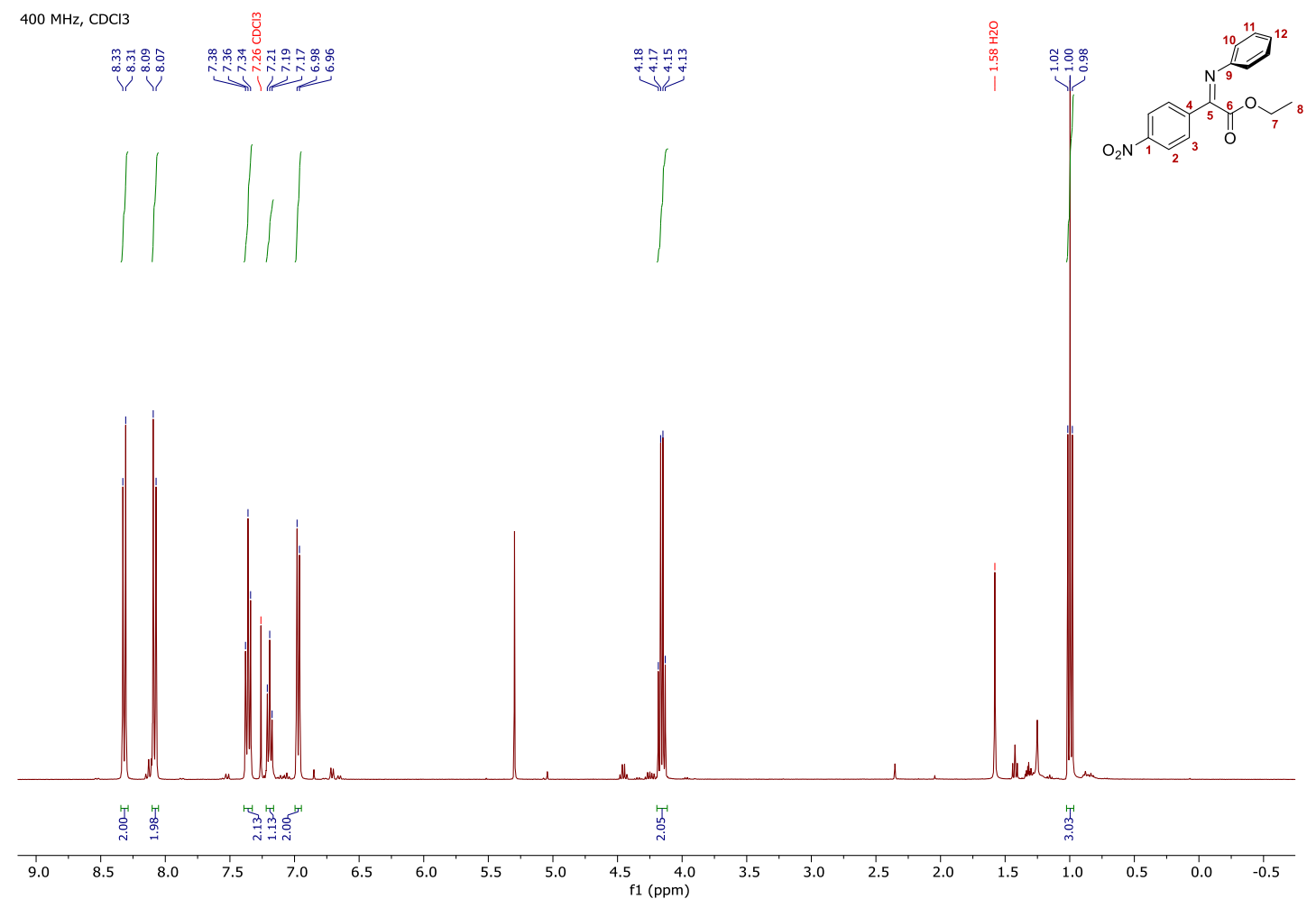

$101 \mathrm{MHz}, \mathrm{CDCl} 3$

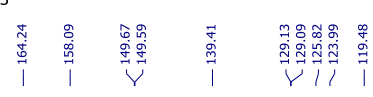



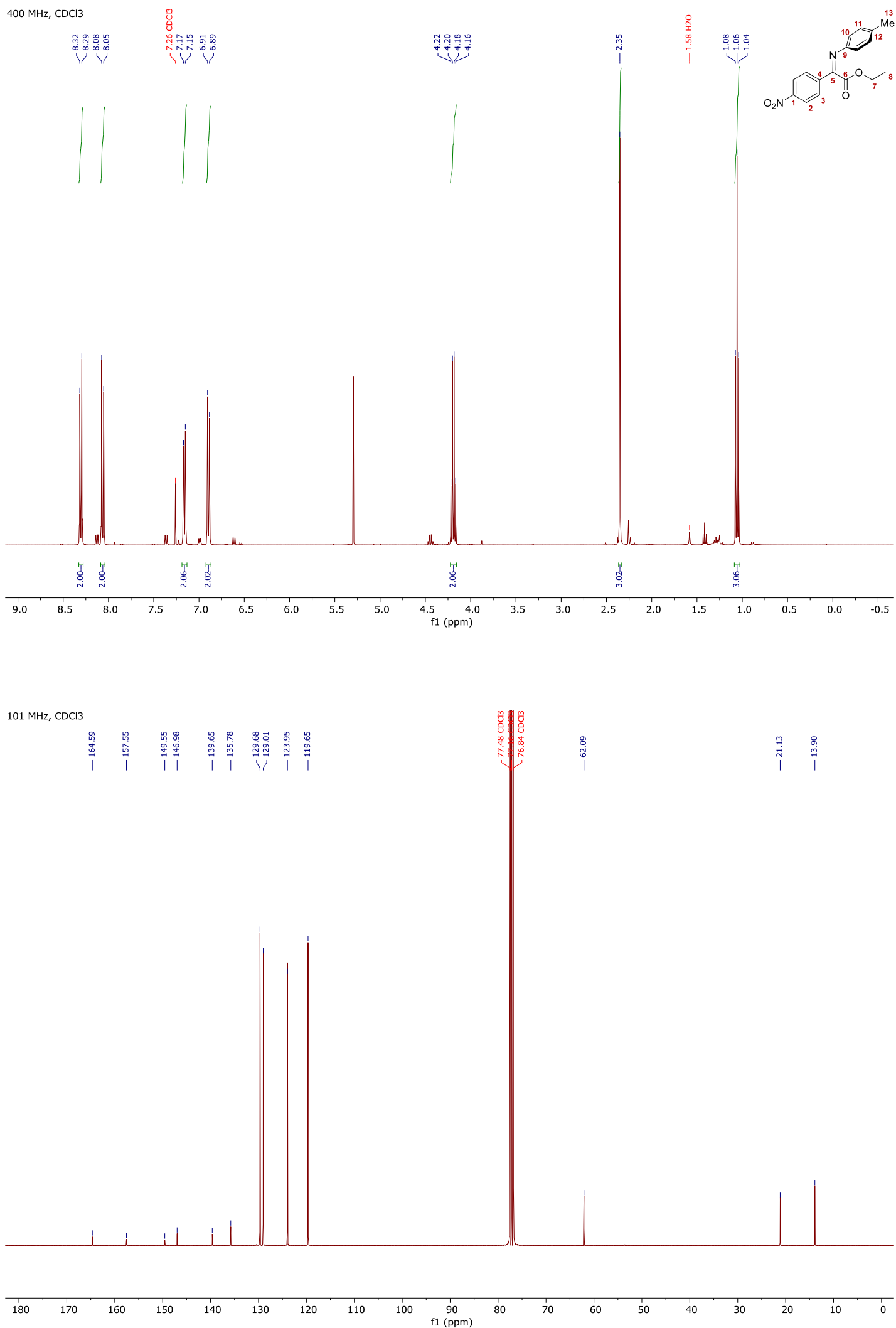
$11 c$

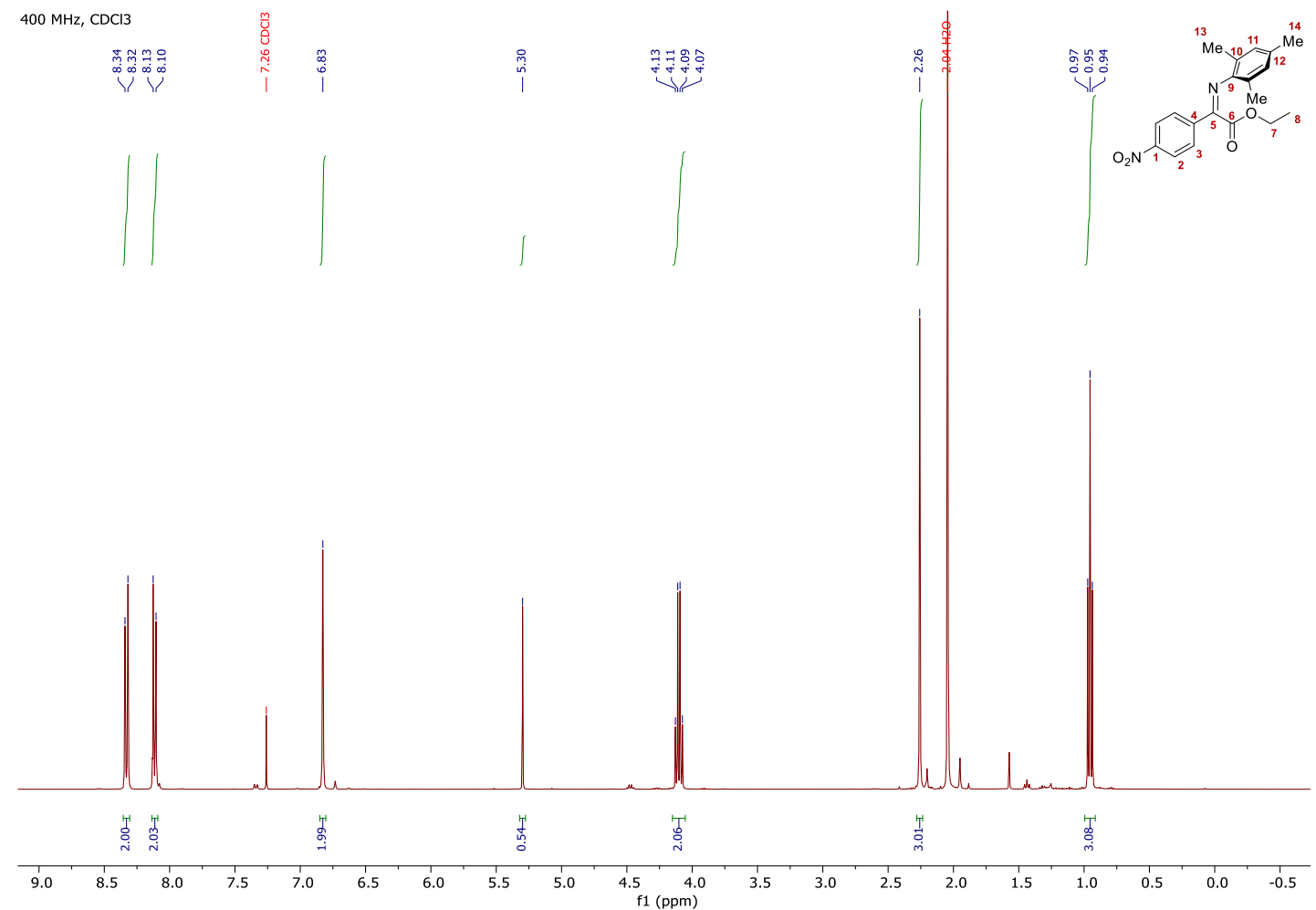

$101 \mathrm{MHz}, \mathrm{CDCl} 3$

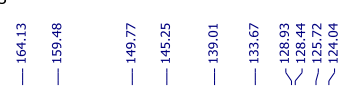

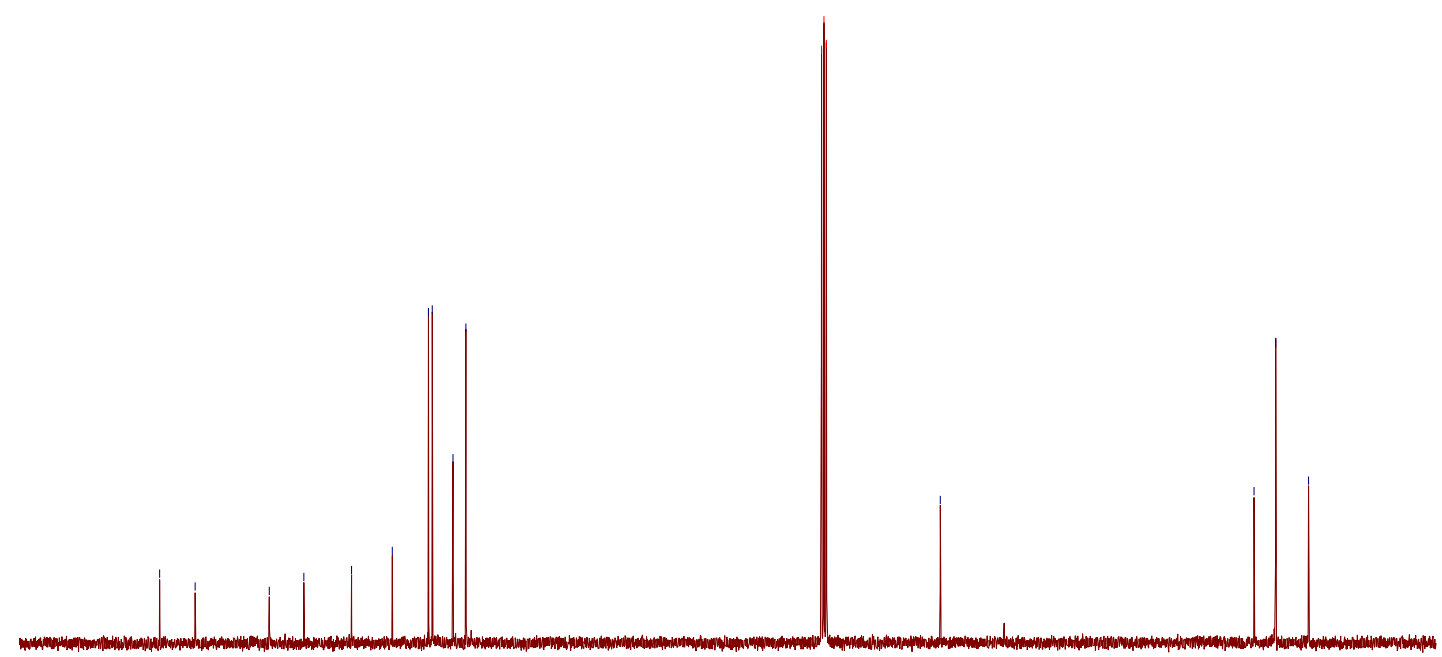

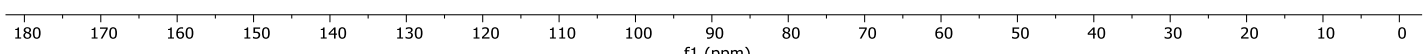


11d

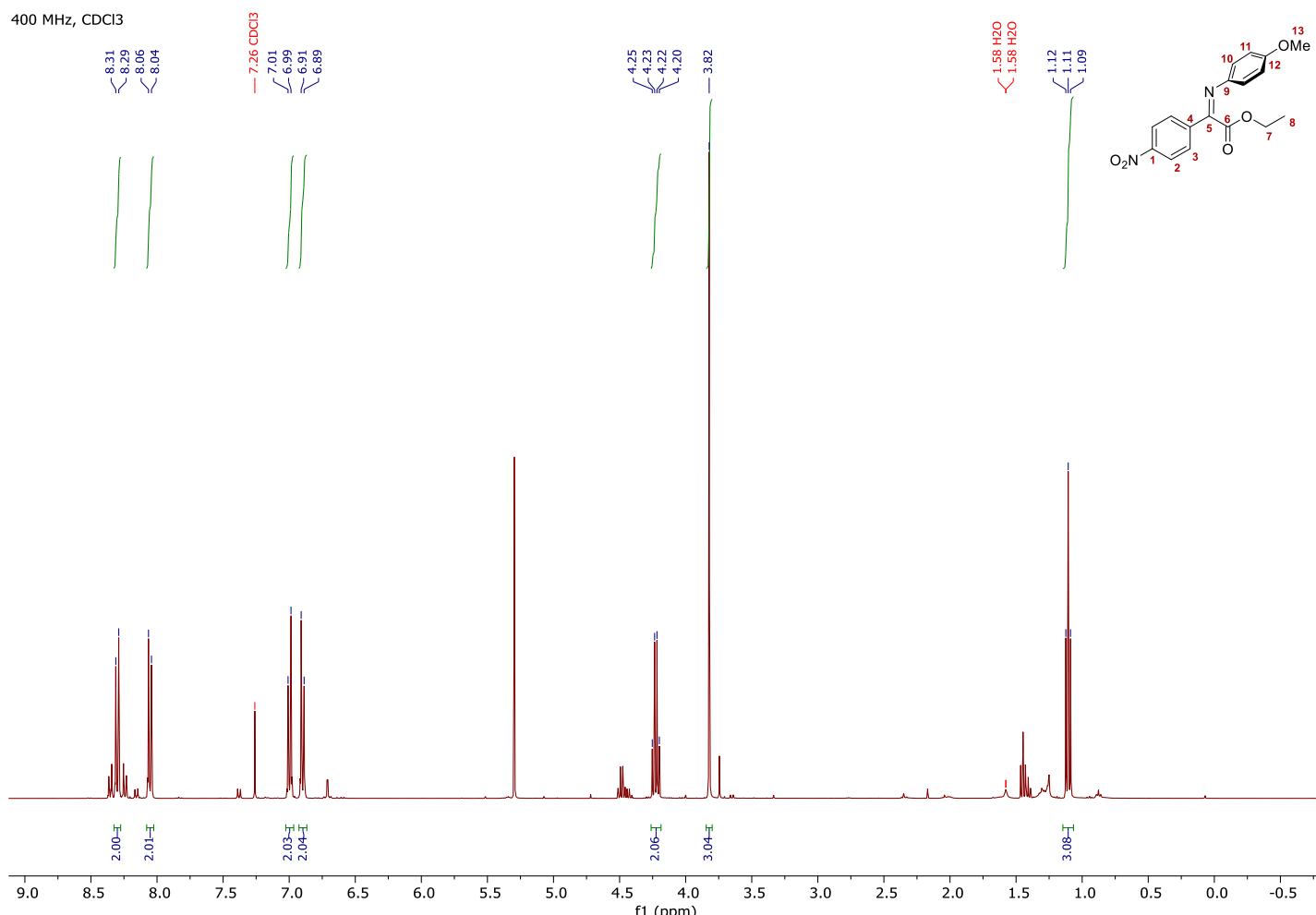

$101 \mathrm{MHz}, \mathrm{CDCl}$

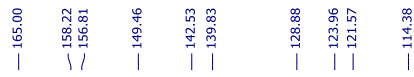

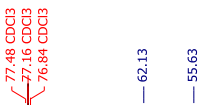

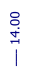

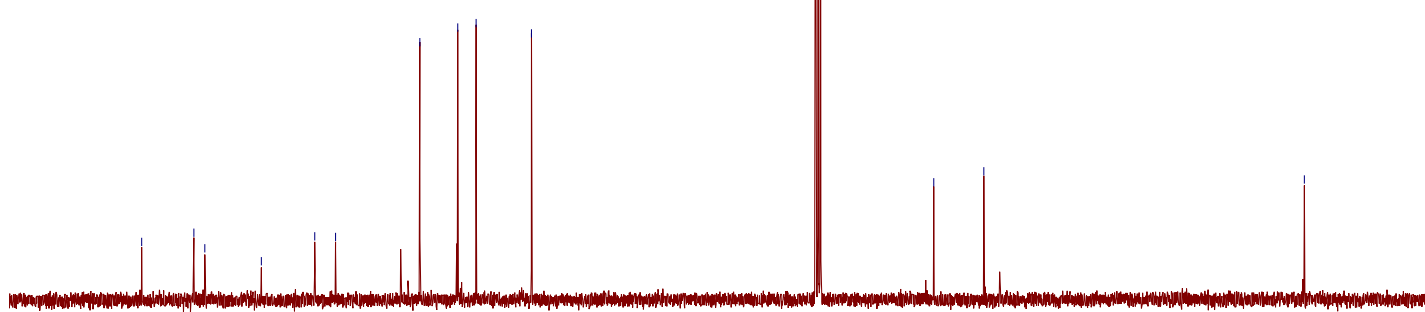

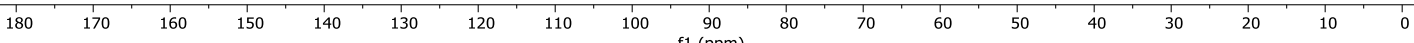


$11 \mathrm{e}$

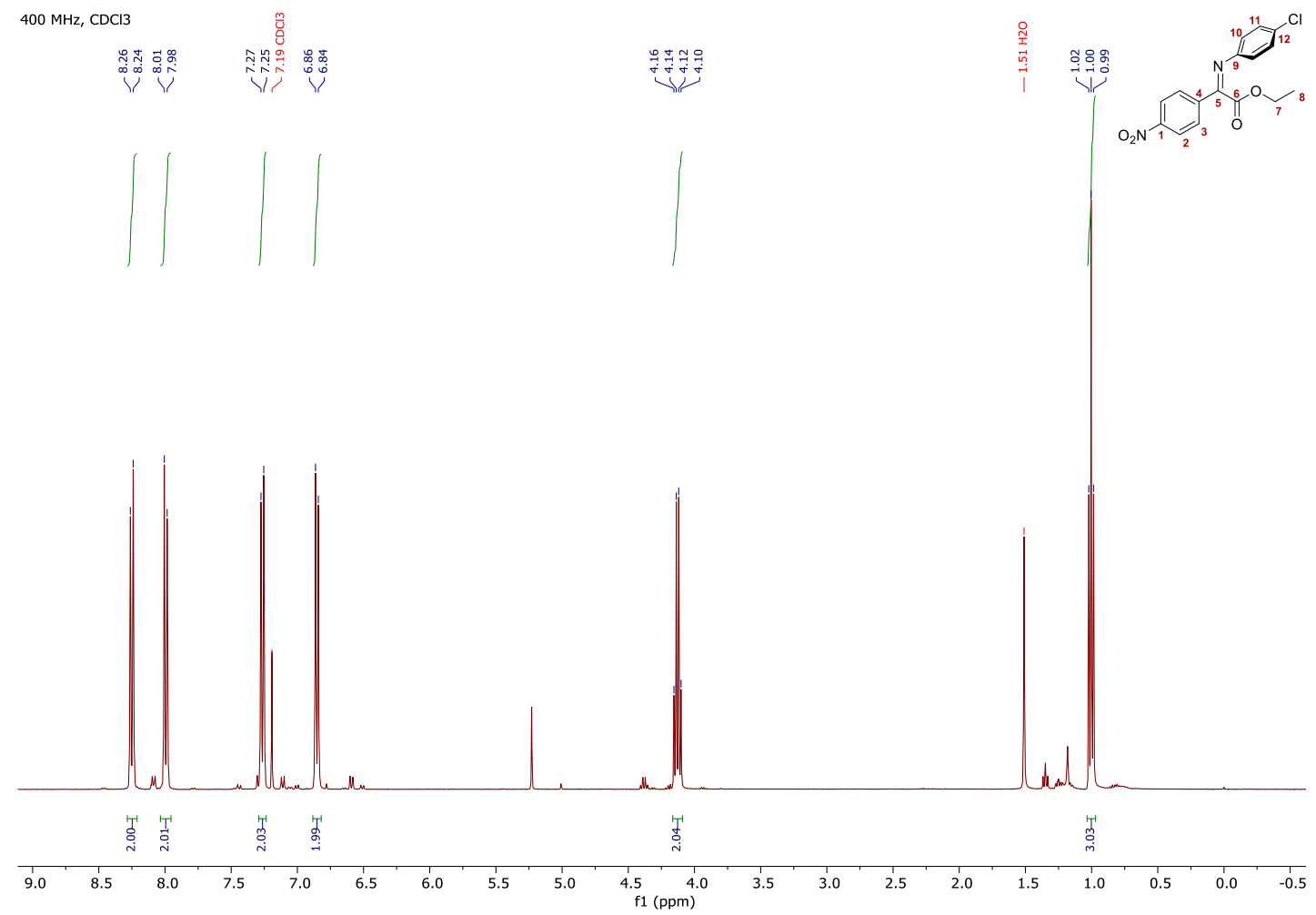

$101 \mathrm{MHz}, \mathrm{CDCl} 3$

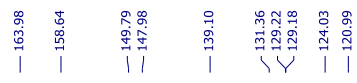
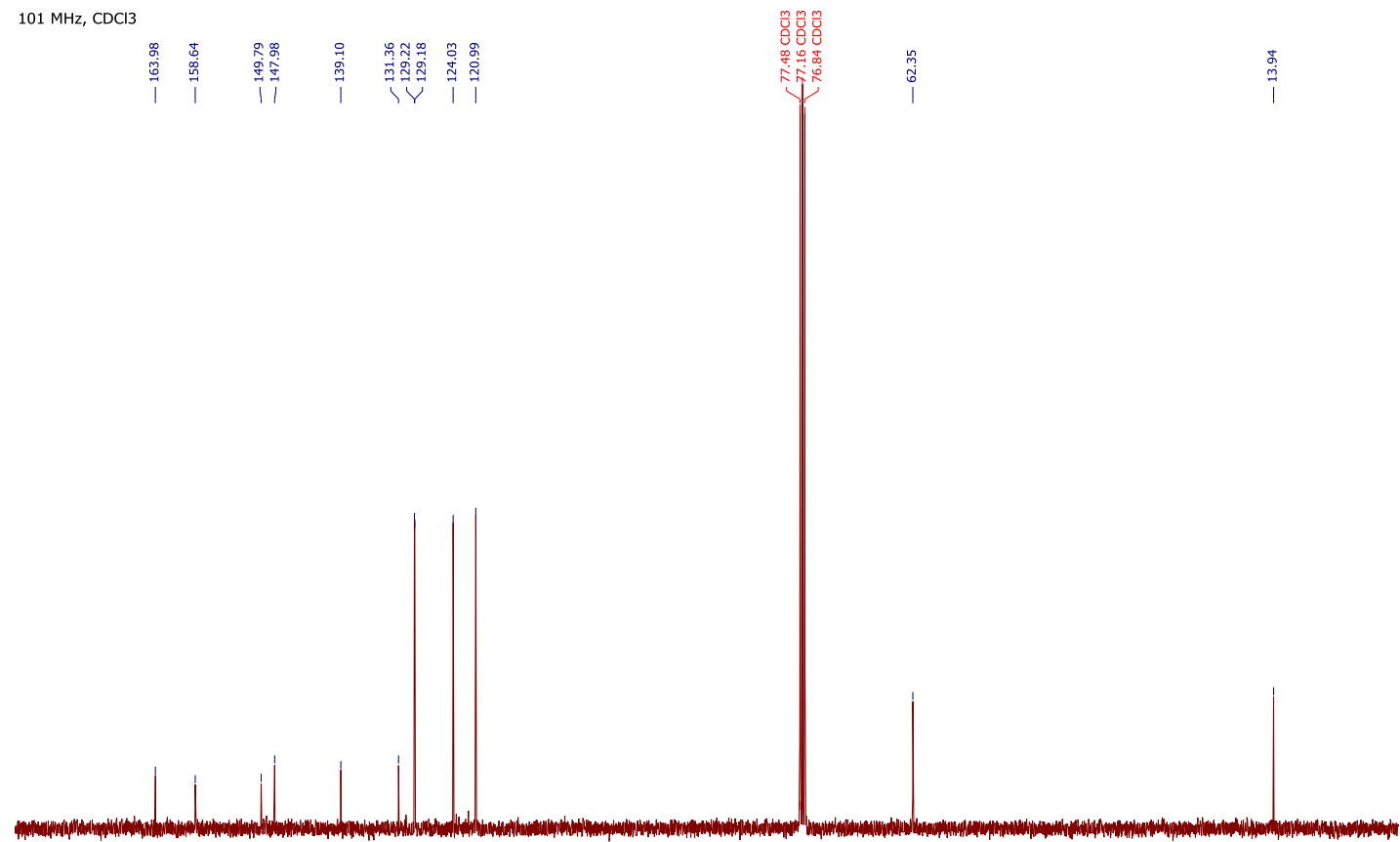

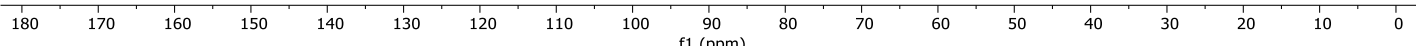




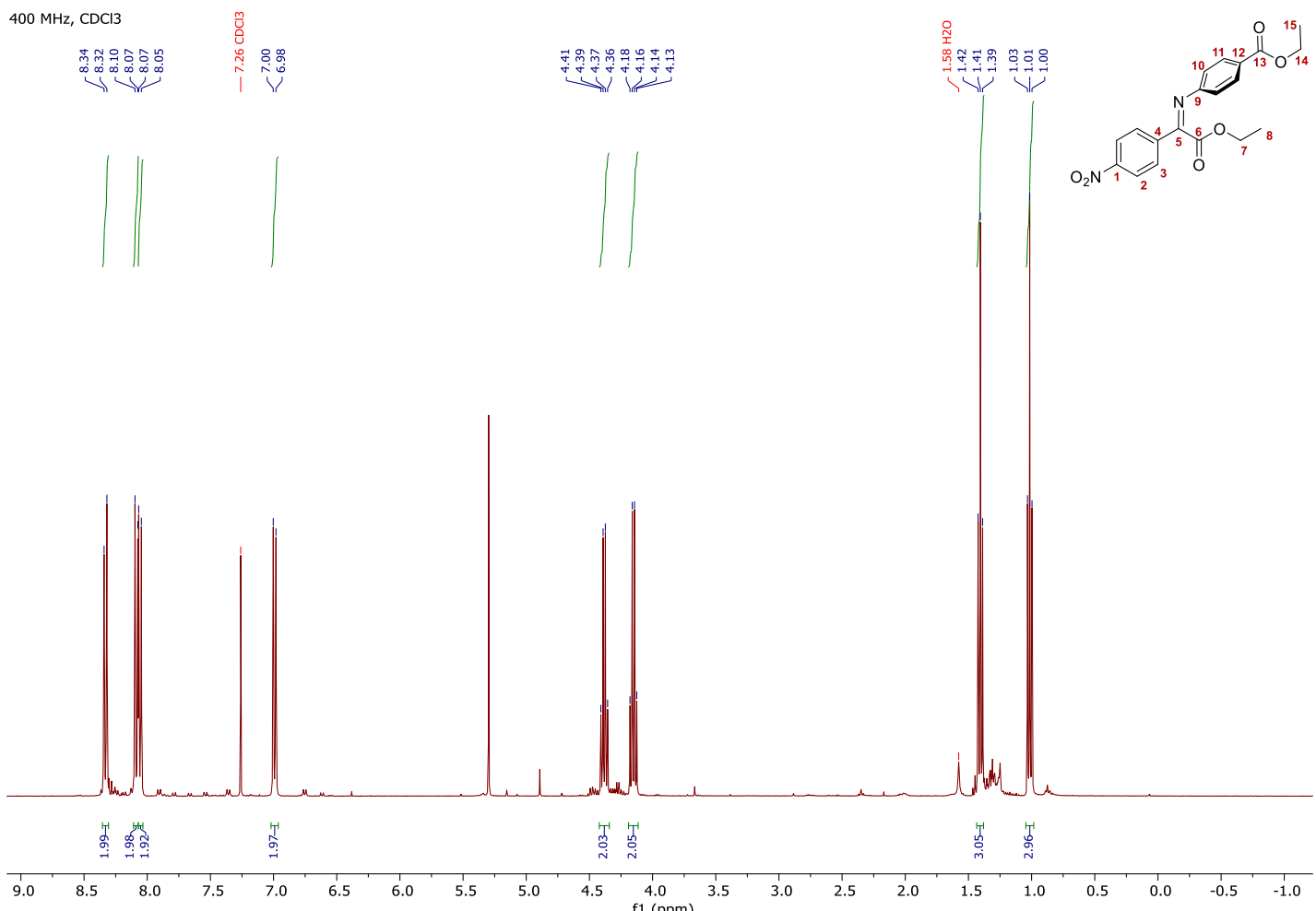

$101 \mathrm{MHz}, \mathrm{CDCl} 3$

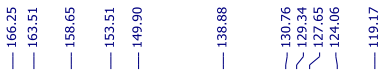

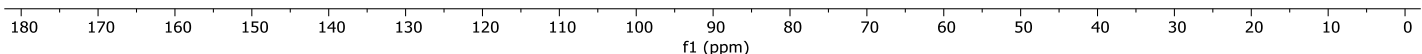



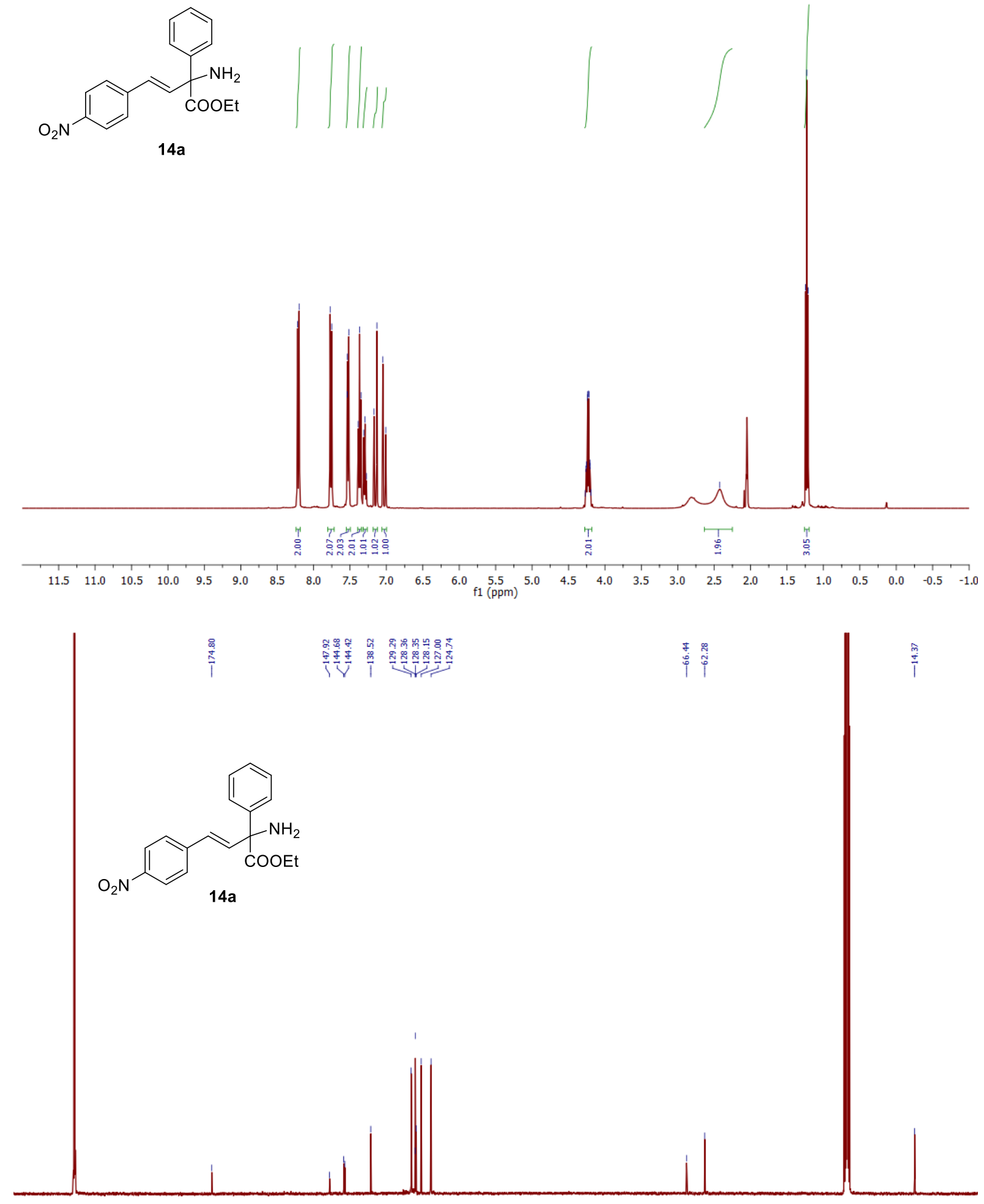

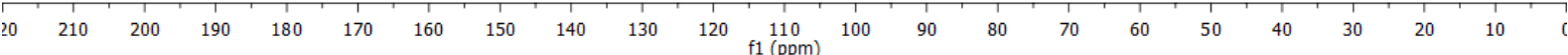



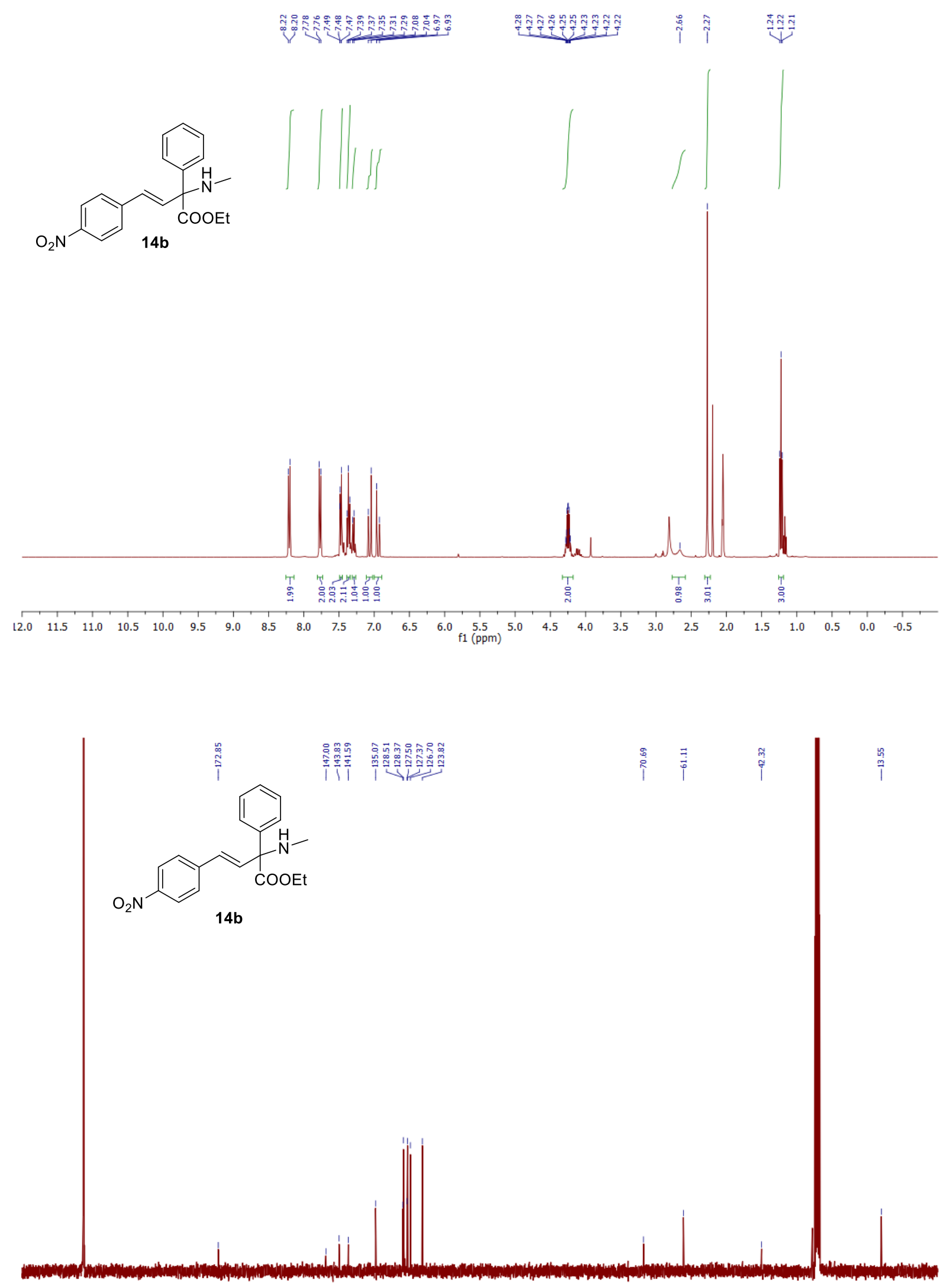

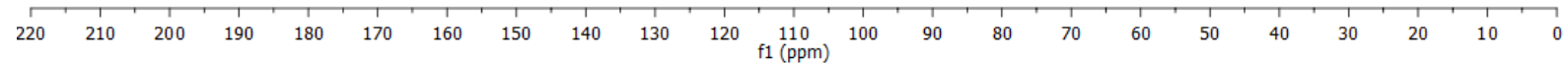




\section{References}

[1] K. Moriyama, Y. Nakamura, H. Togo, Org. Lett. 2014, 16, 3812-3815.

[2] A. J. Brockway, C. C. Cosner, O. A. Volkov, M. A. Phillips, J. K. De Brabander, Synthesis 2016, 48, 2065-2068.

[3] S. W. Youn, T. Y. Ko, Y. H. Jang, Angew. Chemie - Int. Ed. 2017, 56, 6636-6640.

[4] H. R. Lawrence, A. Kazi, Y. Luo, R. Kendig, Y. Ge, S. Jain, K. Daniel, D. Santiago, W. C. Guida, S. M. Sebti, Bioorganic Med. Chem. 2010, 18, 5576-5592.

[5] Y. Ding, S.-Y. Zhang, Y.-C. Chen, S.-X. Fan, J.-S. Tian, T.-P. Loh, Org. Lett. 2019, 21, 2736-2739.

[6] S. Shekhar, T. B. Dunn, B. J. Kotecki, D. K. Montavon, S. C. Cullen, J. Org. Chem. 2011, 76, 4552-4563.

[7] C. M. Holden, S. M. A. Sohel, M. F. Greaney, Angew. Chemie - Int. Ed. 2016, 55, 2450-2453.

[8] F. G. Bordwell, A. B. Colbert, and B. Alan, J. Am. Chem. Soc. 1946, 68, 1778.

[9] B. Pete, G. Simig, L. Poszavacz, L. Toeke, Heterocycles 2003, 60, 2441-2455.

[10] G. Fang, H. Wang, Q. Liu, X. Cong, X. Bi, Asian J. Org. Chem. 2018, 7, 1066-1070.

[11] J. Mangas-Sánchez, E. Busto, V. Gotor-Fernández, V. Gotor, Catal. Sci. Technol. 2012, 2, 15901595.

[12] D. M. Nickerson, V. V. Angeles, T. J. Auvil, S. S. So, A. E. Mattson, Chem. Commun. 2013, 49, 4289-4291. 